Aus der Abteilung Ethik und Geschichte der Medizin

(Prof. Dr. med. C. Wiesemann)

im Zentrum Psychosoziale Medizin

der Medizinischen Fakultät der Universität Göttingen

\title{
Medizinethische Aspekte der fallpauschalisierten Abrechnung im deutschen Krankenhauswesen
}

Eine exemplarische Untersuchung an Patienten mit Bronchialkarzinom in Südniedersachsen für die Jahre 2003-2005

\section{Inaugural-Dissertation}

zur Erlangung des Doktorgrades

der Medizinischen Fakultät

der Georg-August-Universität zu Göttingen

\author{
vorgelegt von \\ Michaela Anders \\ aus \\ Gronau/Leine
}

Göttingen 2009 
Dekan: Prof. Dr. med C. Frömmel
I. Berichterstatter: PD Dr. phil. C. Lenk
II. Berichterstatter: Prof. Dr. med. Trümper
III. Berichterstatter: Prof. Dr. med. Criée

Tag der mündlichen Prüfung: $\quad$ 26. Oktober 2010 


\section{INHALTSVERZEICHNIS}

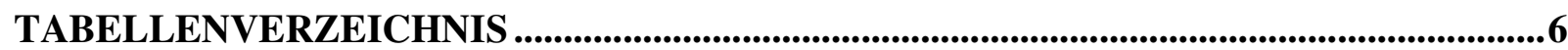

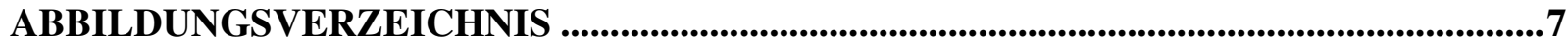

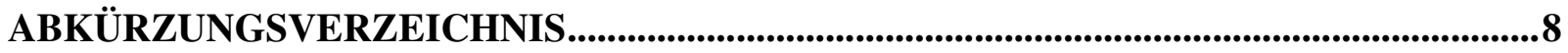

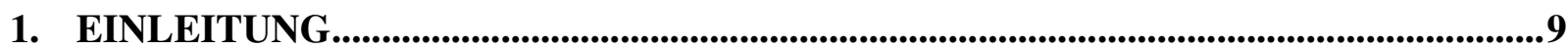

1.1. Abrechnungssysteme im deutschen Gesundheitswesen ............................................11

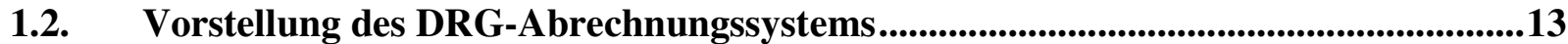

1.2.1. Berechnung des Fallerlöses eines Krankenhausaufenthalts ......................................14

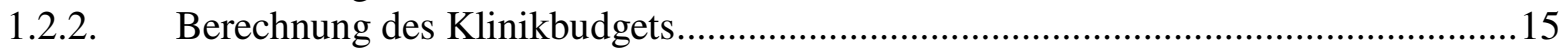

1.3. Fallpauschalen als politisches Steuerungsinstrument ....................................................16

1.4. Erfahrungen mit DRG-Abrechnungssystemen in anderen Ländern...........................17

1.4.1. Durchschnittliche Verweildauer von Patienten im Krankenhaus .............................18

1.4.2. Anzahl der Überweisungen in stationäre Behandlung .............................................19

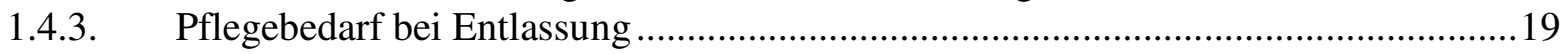

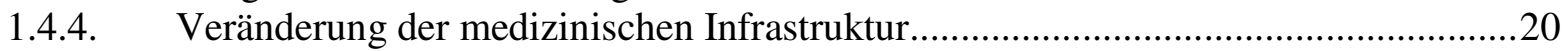

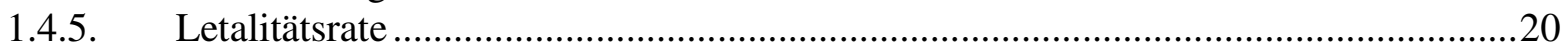

1.4.6. Behandlungsqualität und Zustand der Patienten zum Zeitpunkt der Entlassung .......21

1.4.7. Zusammenfassung der Studienergebnisse ..........................................................21

1.5. Gefahren der DRG-Einführung in Deutschland aus ethischer Perspektive .............22

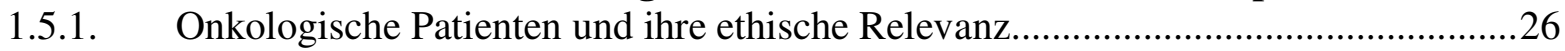

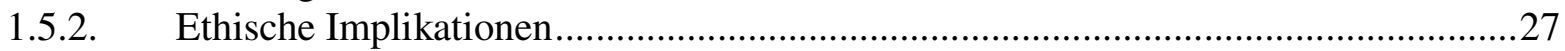

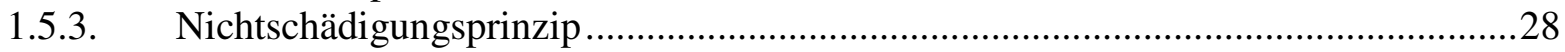

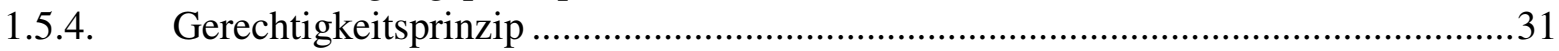

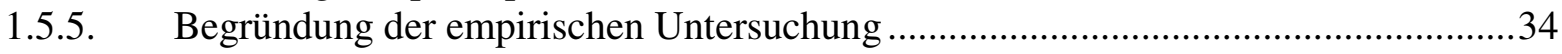

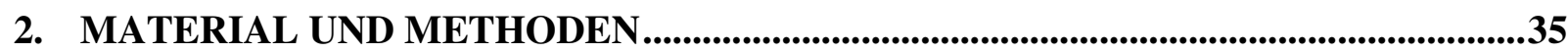

2.1. Allgemeines ....................................................................................................................................35

2.2. Durchführung der Patientenbefragung ....................................................................35

2.2.1. Wahl des Zeitpunktes der empirischen Datenerhebung und Einschlusskriterien der

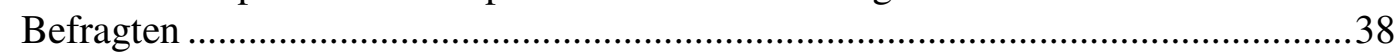

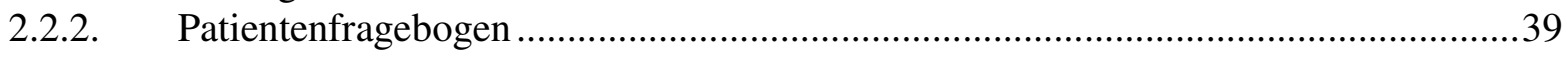




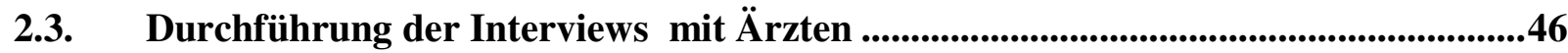

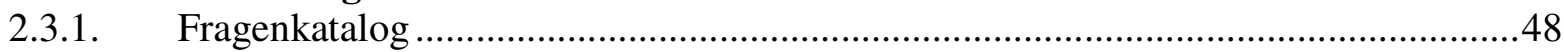

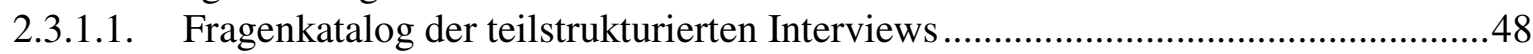

2.4. Durchführung der Interviews mit Pflegekräften .................................................................50

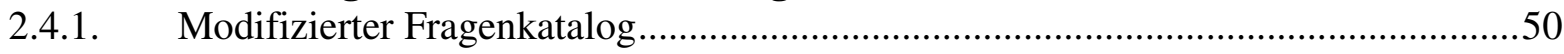

2.5. Erfassung der Controlling-Daten des Universitätsklinikums Göttingen....................51

3. DARSTELLUNG DER UNTERSUCHUNGS-ERGEBNISSE ....................................53

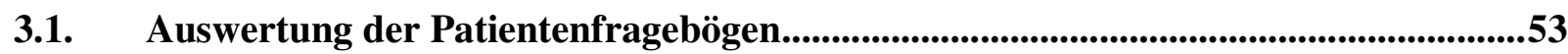

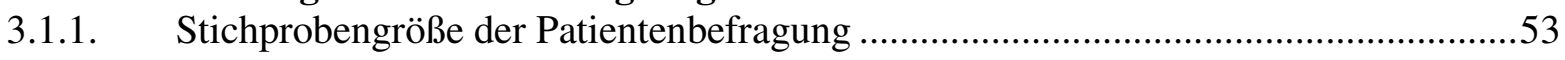

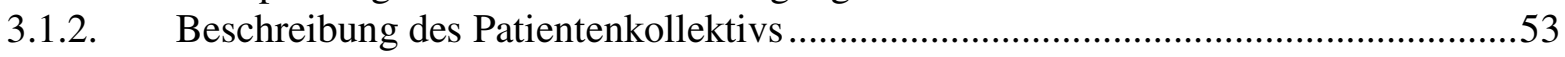

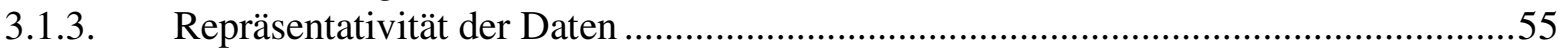

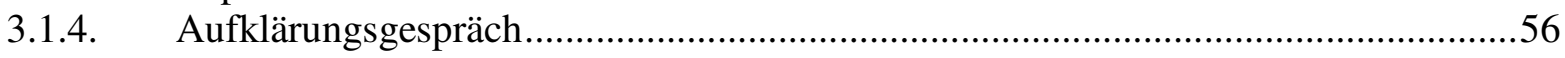

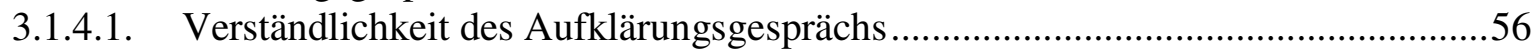

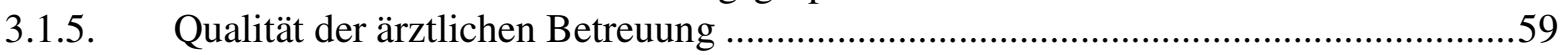

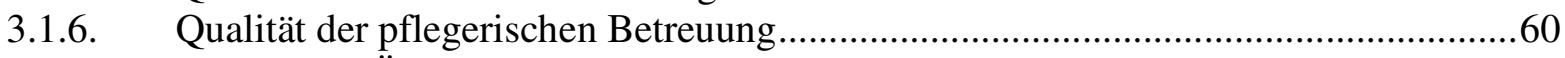

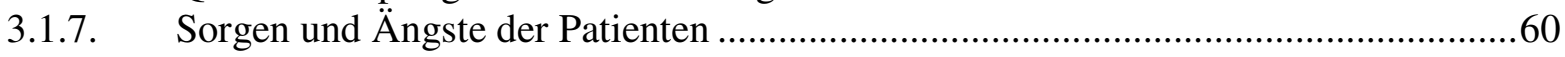

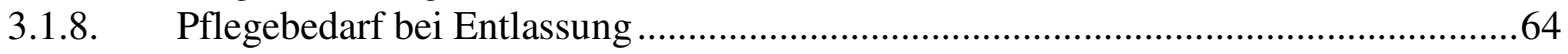

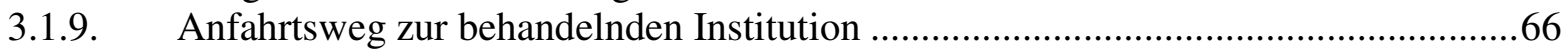

3.1.10. Lebensqualität zum Zeitpunkt der Befragung .....................................................67

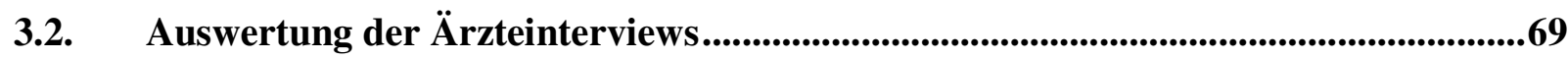

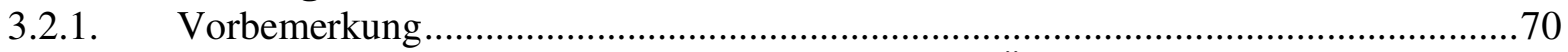

3.2.2. Auswertung der soziodemographischen Daten der Ärzte ...................................... 70

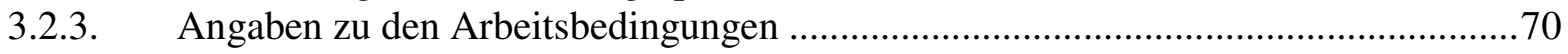

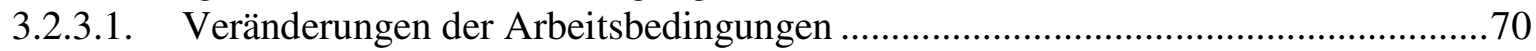

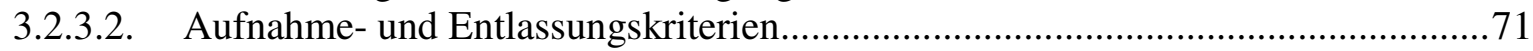

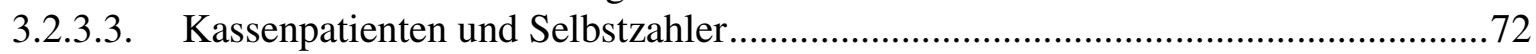

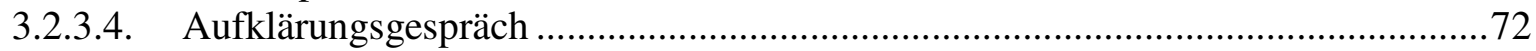

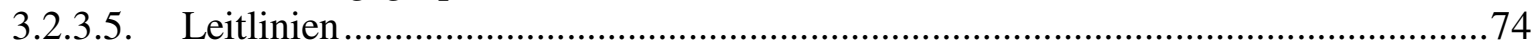

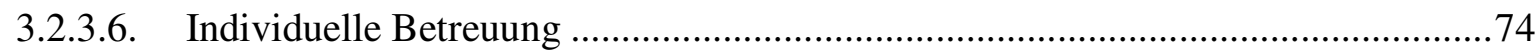

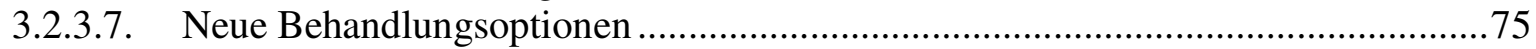

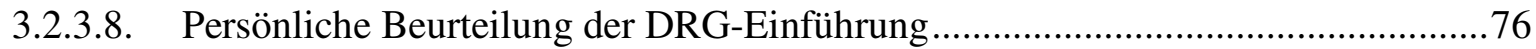

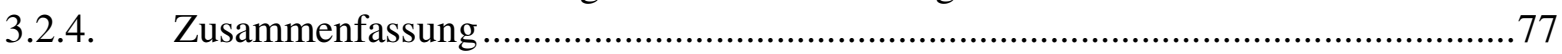

3.3. Auswertung der Befragung von Pflegekräften......................................................78

3.3.1. Auswertung der soziodemographischen Daten der Pflegekräfte ..............................78

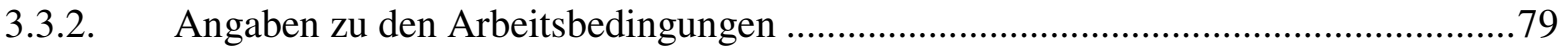

3.3.2.1. Veränderungen der Arbeitsbedingungen und Zufriedenheit ...................................79

3.3.2.2. Das DRG-System als Diskussionsthema der Pflegekräfte .................................... 80

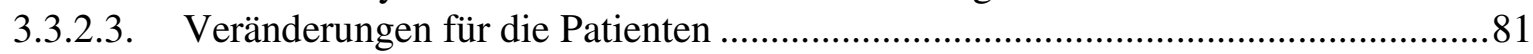

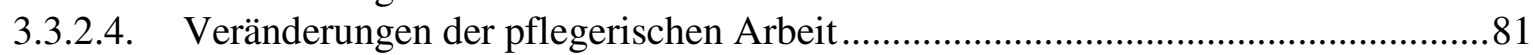

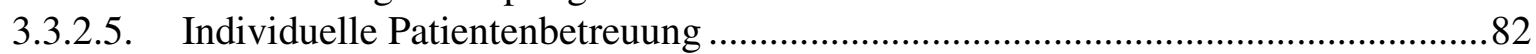

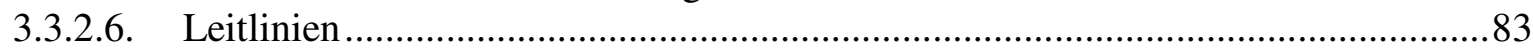




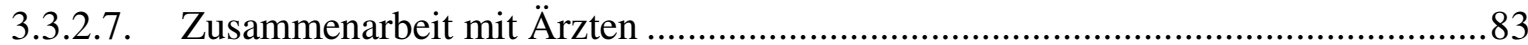

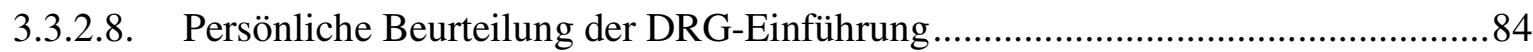

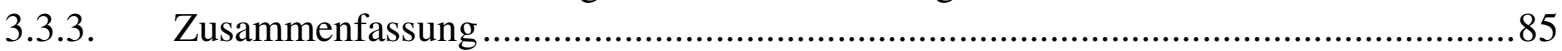

3.4. Auswertung der Controlling-Daten des Universitätsklinikums Göttingen .................86

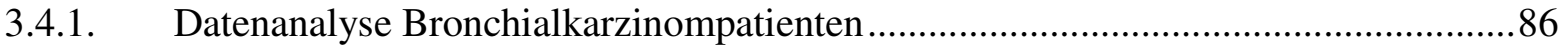

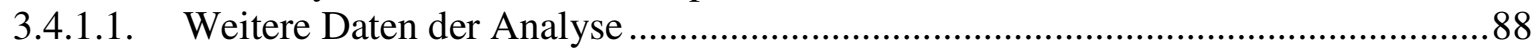

3.5. Ergebniszusammenfassung anhand der Forschungsfragen........................................91

3.5.1. Gibt es aus Sicht der Studienteilnehmer eine Veränderung in der

Behandlungsqualität von Bronchialkarzinompatienten während der DRG-

Einführung?

3.5.2. Verändert sich die Lebensqualität der Patientenpopulation während der Einführung

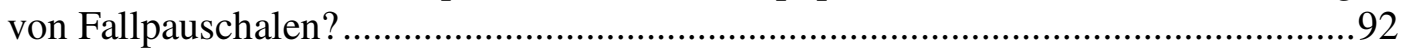

3.5.3. Verändert sich der Pflegebedarf der Patienten bei Entlassung? ................................93

3.5.4. Verändert sich die stationäre Letalitätsrate der Bronchialkarzinompatienten während der Einführung des DRG-Systems? .......................................................................94

3.5.5. Kommt es aus Sicht der Studienteilnehmer zu Einschränkungen in der Therapie- und Behandlungsfreiheit von Bronchialkarzinompatienten? ...........................................94

3.5.6. Können Fallpauschalen individuell auftretende Probleme erfassen und kann ein Patient seinem psychischen und physischen Gesundheitszustand entsprechend behandelt werden? .........................................................................................99

3.5.7. Haben Patienten, die in ländlichen Regionen wohnen, einen fairen Zugang zu ihrer medizinischen Versorgung?..................................................................................97

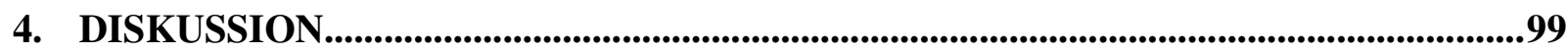

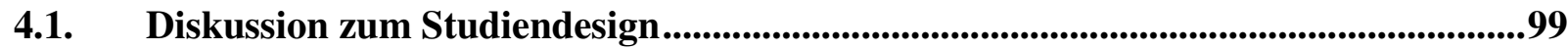

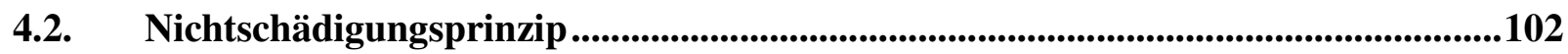

4.3. Gerechtigkeit .....................................................................................................................111

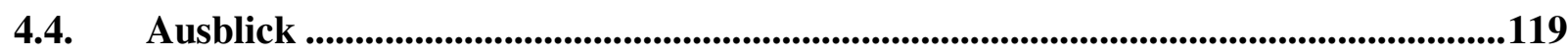

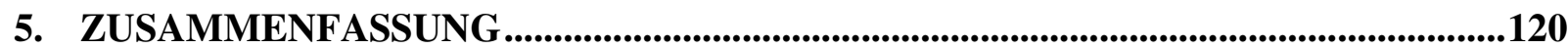

6. ANHANG: DAS FALLPAUSCHALENGESETZ UND SEINE ÄNDERUNGEN..........126

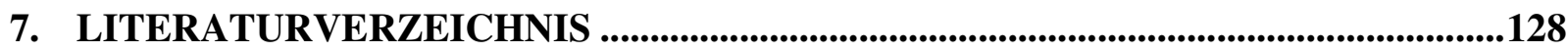

Anmerkung: In dieser Dissertation wurde auf die Aufzählung beider Geschlechter (die Ärzte/ Ärztinnen) oder die Verbindung beider Geschlechter in einem Wort (ÄrztInnen) zugunsten einer möglichst flüssigen Lesart des Textes verzichtet. Die Nennung nur der männlichen Form bei Berufsgruppen schließt die jeweils weiblichen Berufstätigen ein. 


\section{TABELLENVERZEICHNIS}

Tabelle 3.1.1 Stichprobenumfang der Patientenbefragung 2003-2005 .......................................53

Tabelle 3.1.2a Vergleich der soziodemographischen Patientendaten 2003-2005 .........................54

Tabelle 3.1.2b Vergleich höchster Bildungsabschluss der Patienten ...........................................54

Tabelle 3.1.4.1a Mittelwerte der Verständlichkeit des Aufklärungsgesprächs 2003-2005 aus

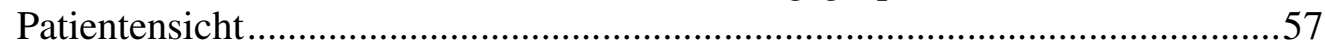

Tabelle 3.1.4.1b Fehlende Erinnerung an eine Aufklärung über Behandlungsweise bzw.

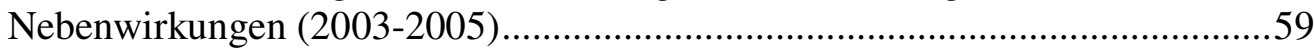

Tabelle 3.1.5 Vergleich der Bewertung der ärztlichen Betreuung durch die Patienten (2003-2005)

Tabelle 3.1.6 Vergleich der Bewertung der pflegerischen Betreuung (2003-2005) ....................60

Tabelle 3.1.7 Angaben über Sorgen und Ängste der Patienten 2003-2005 im Vergleich.............61

Tabelle 3.1.8 Pflegebedarf der Patienten bei Entlassung im Vergleich 2003-2005 .....................65

Tabelle 3.1.10a Auswertung der Fragebögen zur Lebensqualität, transformierte Daten aller drei Jahre im Vergleich

Tabelle 3.1.10b Vergleich der transformierten Funktions- und Symptommittelwerte von 20032005

Tabelle 3.4.1a Fallzahl, mittlere Verweildauer und Krankenhausletalität der Diagnose "Bronchialkarzinom" des Universitätsklinikums Göttingen 2003-2005 . .86

Tabelle 3.4.1b Aufteilung der Fälle in operative und konservative Fälle im Vergleich 2003-2005

Tabelle 3.4.1c Vergleich der mittleren Verweildauer für operative und konservative DRG 2004 und 2005

Tabelle 3.4.1d Vergleich der Daten des Controllings (Kurzlieger, Langlieger, Relativgewicht, Effektivgewicht, Effektiverlös und Erlösanteil an Zusatzentgelten) für die Jahre 2003-2005 


\section{ABBILDUNGSVERZEICHNIS}

Abbildung 1.2.2. Erläuterungen zur Berechnung des Klinikbudgets

Abbildung 1.5. Anzahl der vorgehaltenen Betten in deutschen Kliniken im

Vergleich 1991 bis 2007 (Quelle: Statistisches Bundesamt 2008)

Abbildung 3.1.4. Mediane der von den Patienten geschätzten Dauer des

Aufklärungsgesprächs im Vergleich 2003-2005

Abbildung 3.1.7a Angaben über Sorgen und Ängste der Patienten 2003-2005 im

Vergleich

Abbildung 3.1.7b Häufigkeit der Patientenangaben über den Klinikarzt als Ansprechpartner bei Sorgen und Ängsten im Vergleich 20032005 .64

Abbildung 3.1.9 Vergleich der Mediane der Länge der tatsächlichen Anfahrtswege und der ,,angemessenen“ Anfahrtswege aus Sicht der Patienten 2003-2005 .66 


\section{ABKÜRZUNGSVERZEICHNIS}

\begin{tabular}{|c|c|}
\hline AR-DRG & Australian Diagnosis-Related Groups \\
\hline $\mathrm{BR}$ & base-rate (Basisfallpreis) \\
\hline bzw. & beziehungsweise \\
\hline ca. & circa \\
\hline $\mathrm{CC}$ & Komplikationen oder Komorbiditäten \\
\hline CMI & Case-Mix-Index \\
\hline DKG & Deutsche Krankenhausgesellschaft \\
\hline DRG & Diagnosis-Related Groups (Fallpauschalen) \\
\hline DVfR & Deutsche Vereinigung für Rehabilitation Behinderter \\
\hline ebd. & ebenda \\
\hline EORTC & $\begin{array}{l}\text { The European Organization for Research and Treatment of } \\
\text { Cancer }\end{array}$ \\
\hline FPÄndG & Fallpauschalenänderungsgesetz \\
\hline FPG & Fallpauschalengesetz \\
\hline G-DRG & German Diagnosis-Related Groups \\
\hline InEK & Institut für das Entgeltsystem im Krankenhaus \\
\hline Kap. & Kapitel \\
\hline LEP & Leistungserfassung in der Pflege \\
\hline Min. & Minute \\
\hline NSCLC & Non small cell lung cancer \\
\hline PET & Positronen-Emissions-Tomographie \\
\hline RG & Relativgewicht \\
\hline RS & Raw-score (errechneter Durchschnittswert) \\
\hline SCLC & Small cell lung cancer \\
\hline s. o. & siehe oben \\
\hline USA & United States of America \\
\hline VWD & Verweildauer \\
\hline z.B. & zum Beispiel \\
\hline
\end{tabular}




\section{EINLEITUNG}

In Deutschland hat sich seit dem Jahr 2004 das Abrechnungssystem der stationären Krankenhausversorgung grundlegend verändert. Das Prinzip des neuen Systems besteht nicht mehr in der Abrechnung nach Tagessätzen, sondern nach Fallpauschalen, sogenannten DRG (ㅁiagnosis-ㅌelated Groups). Das DRG-System ordnet einer Erkrankung ein bestimmtes Honorar zu. Dieses Honorar ist unabhängig von der individuell erbrachten Leistung und der Liegedauer der Patienten. Die deutsche Bundesregierung hat die Einführung dieses Systems im Jahr 2000 im Rahmen der Gesundheitsversorgungsreform beschlossen, um eine effizientere Versorgung im stationären Gesundheitswesen zu erreichen. ${ }^{1}$

Das DRG-System hat seinen Ursprung in den USA und wird bereits in vielen Ländern als Abrechnungsmodus von Kliniken verwendet. Deutschland ist das erste Land, in dem alle stationären Behandlungen, die an Patienten im Krankenhaus vorgenommen werden (mit Ausnahme der Psychiatrie), durch DRG vergütet werden. Das System schafft Anreize, Patienten möglichst effizient, kostengünstig und zügig stationär zu behandeln.

Vor und während der DRG-Einführung wurde in Deutschland eine Diskussion über die Folgen eines solchen Abrechnungssystems geführt. Gesundheitsexperten, Mediziner und Ethiker äußerten Befürchtungen, dass sich die Behandlungsqualität vermindern und bestimmte Patientengruppen benachteiligt werden könnten. Diese Sorge begründet sich darin, dass das DRG-System Anreize setzt, den Versorgungsaufwand möglichst gering zu halten. Eine ethische Auseinandersetzung mit dem Thema Fallpauschalen ist deshalb wichtig, weil zu erwarten ist, dass die DRG die Rahmenbedingungen von stationären Behandlungen wesentlich prägen werden. Diese Rahmenbedingungen werden die medizinische Behandlung und Pflege konkret beeinflussen (Wehkamp 2002).

\footnotetext{
1 Ziele der Bundesregierung waren: „mehr Transparenz und Vergleichbarkeit der Krankenhausleistungen, Optimierung der Behandlungsabläufe, Abbau von Überversorgung, Verkürzung der durchschnittlichen vollstationären Aufenthaltszeit und Begrenzung zu erwartender Kostensteigerungen im stationären Bereich“ (Deutsche Bundesregierung 2007, S.1).
} 


\section{EINLEITUNG}

Die Ziele und Anreize werden in der vorliegenden Studie unter ethischen Aspekten exemplarisch an Patienten mit Bronchialkarzinom untersucht. Dabei sollen Veränderungen sichtbar gemacht werden und anschließend aus der Perspektive der ethischen Prinzipien des Nichtschädigens und der Gerechtigkeit untersucht werden, denn auch unter veränderten Rahmenbedingungen ist es wichtig, dass den Patienten durch das Abrechnungssystem kein Schaden zugefügt wird und dass das System nicht zu einer ungerechten Behandlung von Patienten führt.

In der vorliegenden Studie werden die möglichen Veränderungen am Beispiel einer betroffenen Patientengruppe sowie auf der Basis von Experteninterviews mit den sie behandelnden Ärzten und Pflegekräften untersucht. In die Auswertung fließen außerdem Daten der Stabsstelle Controlling des Göttinger Universitätsklinikums ein. Die untersuchte Patientenpopulation besteht aus schwerkranken Patienten, denn besonders für diese Gruppe wird von Seiten der Gesundheitsexperten eine Verschlechterung der Behandlungsqualität befürchtet.

In der vorliegenden Arbeit wird zunächst das DRG-Abrechnungssystem vorgestellt. Dann werden die von politischer Seite beabsichtigten Veränderungen beschrieben. Es schließt sich eine Darstellung von Studienergebnissen aus den USA an. Diese Studien wurden in den USA während der dortigen DRG-Einführung durchgeführt und können Hinweise zu möglichen systembedingten Veränderungen geben. Aus diesen Ergebnissen und den von Kritikern erwarteten Veränderungen werden forschungsleitende Fragen generiert, die mit Hilfe der empirischen Ergebnisse beantwortet werden sollen.

Es folgt der empirische Teil dieser Arbeit. Er gliedert sich in vier Abschnitte. Der erste Abschnitt befasst sich mit den Veränderungen während der DRG-Einführung aus Patientensicht. Dazu wurden in drei aufeinander folgenden Jahren (2004-2006) Fragebögen an Patienten mit Bronchialkarzinom versandt, die im jeweils vorangegangenen Jahr aufgrund ihrer Erkrankung in stationärer Behandlung waren. Die Patienten beurteilen jeweils den Klinikaufenthalt im vorangegangenen Jahr. Anhand dieser Antworten können die Bedingungen der stationären Behandlung aus Sicht der befragten Patienten vor und während der DRG-Einführung miteinander verglichen und Veränderungen sichtbar gemacht werden.

Im zweiten empirischen Abschnitt werden die Ergebnisse von teilstrukturierten Interviews mit Ärzten vorgestellt. Die Befragung fand zwischen November 2005 und Januar 2006 statt, nachdem die befragten Ärzte zum Interviewzeitpunkt rund zwei Jahre mit dem neuen 
Abrechnungssystem gearbeitet hatten und Veränderungen des Arbeitsalltags, die sich daraus ergeben hatten, beurteilen konnten. Außerdem wurden die Ärzte zu ihrer persönlichen Beurteilung des DRG-Systems befragt.

Im dritten empirischen Teil dieser Arbeit wurden analog zum zweiten Abschnitt Interviews mit Pflegekräften geführt. Die Erhebung fand im April 2006 statt.

Im letzten Abschnitt der empirischen Erhebung wurden Daten der Stabsstelle Controlling des Göttinger Universitätsklinikums erhoben und ausgewertet, die sich auf die stationäre Behandlung der Patienten mit Bronchialkarzinom in der Zeit von 2003-2005 bezogen.

Die Ergebnisse der vier empirischen Untersuchungen werden dann im folgenden Abschnitt zur Beantwortung der forschungsleitenden Fragen herangezogen und aus der Perspektive der ethischen Prinzipien des Nichtschädigens und der Gerechtigkeit untersucht. In der abschließenden Diskussion werden die Ergebnisse mit den beiden ethischen Prinzipien in Beziehung gesetzt und bewertet.

\subsection{Abrechnungssysteme im deutschen Gesundheitswesen}

In den letzten Jahren ist es durch verschiedene demographische, medizinische und administrative Faktoren zu einer Steigerung der Ausgaben im Gesundheitswesen gekommen:

- Eine verbesserte medizinische Versorgung führt zu einem Anstieg des durchschnittlichen Lebensalters der Bevölkerung und zu einem höheren Anteil älterer und multimorbider Patienten in der stationären Versorgung (Buchner und Wasem 2000);

- es gibt neue, leistungsfähigere diagnostische und therapeutische Verfahren, die kostenintensiver als bisherige Behandlungsmethoden sind;

- der Investitionsbedarf für den medizinischen Fortschritt und den Bauunterhalt der benötigten Immobilien steigt an (Leititis 2000).

Die steigenden Kosten der Krankenhausbehandlungen ließen sich nicht mehr mit den Einnahmen der Krankenversicherungen decken. Um eine Finanzierung des deutschen Gesundheitswesens weiterhin sicherzustellen, hat die Bundesregierung im Jahr 2000 im Rahmen einer umfassenden 


\section{EINLEITUNG}

Gesundheitsversorgungsreform die Einführung von Fallpauschalen beschlossen. So soll eine effizientere Versorgung erreicht werden, um Kosten zu reduzieren. Das Bundesministerium für Gesundheit schreibt in seiner Begründung zum Fallpauschalengesetz, „dass die Ressourcen krankenhausintern wie auch krankenhausübergreifend bedarfsgerechter und effizienter eingesetzt werden [sollen]“ (Bundesministerium für Gesundheit 2001, S.2).

Bis Dezember 2003 wurde die Vergütung von stationären Behandlungen auf der Basis von „tagesgleichen Pflegesätzen“ errechnet. Ein Krankenhaus bekam von den Krankenkassen für jeden Tag des stationären Aufenthalts einen bestimmten Betrag. Die Einnahmen der Kliniken hingen also unmittelbar von der durchschnittlichen Verweildauer der Patienten ab. Für die Krankenhäuser gab es einen ökonomischen Anreiz, die Patienten möglichst lange stationär zu behandeln. Die Pflegesätze wurden durch Sonderentgelte und Fallpauschalen, die für „Routinebehandlungen“, wie zum Beispiel unkomplizierte Operationen, veranschlagt wurden, ergänzt.

Seit dem Jahr 2004 erfolgte schrittweise eine Ablösung des alten Abrechnungssystems durch das DRG-System. Bereits im Jahr 2003 konnten Kliniken optional ihre Abrechnung auf der Basis der DRG durchführen (sogenanntes „Optionsmodell“). Diese Art der Abrechnung verlief parallel zur Vergütung nach „tagesgleichen Pflegesätzen“ und war für die Kliniken zunächst kostenneutral. In der Einführungszeit, der sogenannten Konvergenzphase, wurden die einzelnen DRG an die tatsächlich in Deutschland anfallenden Kosten angeglichen. Eine schrittweise Anpassung war wichtig, damit auch während der Systemumstellung ein reibungsloser Ablauf der Krankenhausversorgung gewährleistet werden konnte. Im Jahr 2004 wurde flächendeckend mit der Kodierung nach DRG begonnen. Ab dem Jahr 2005 wurde das Klinikbudget anteilig mit Hilfe der DRG berechnet. Der übrige Anteil wurde weiterhin über das alte System der Pflegesätze errechnet. Zunächst musste jede Klinik intern die durchschnittlichen Kosten (Basisfallpreis) für eine stationäre Behandlung berechnen. Im Rahmen der Konvergenzphase wurde der klinikinterne Basisfallpreis an einen bundesland-einheitlichen Basisfallpreis angeglichen. Dadurch wurde erreicht, dass am Ende der Konvergenzphase in jedem Bundesland einheitliche Beträge für eine bestimmte Fallpauschale gezahlt werden (s. Kap. 1.2.). Die Konvergenzphase, deren Dauer ursprünglich bis zum 1. Januar 2007 vereinbart worden war, wurde im Rahmen des 2. Fallpauschalenänderungsgesetzes (s. Anhang) bis zum Jahr 2009 


\section{EINLEITUNG}

verlängert. Seit Januar 2009 erfolgt eine vollständige Abrechnung der Krankenhausbehandlungen nach bundesland-einheitlichen Basisfallpreisen. Das hat zur Folge, dass in jedem deutschen Bundesland für die stationäre Behandlung einer bestimmten Diagnose ein einheitlicher Preis gilt.

\subsection{Vorstellung des DRG-Abrechnungssystems}

Auch in Australien, Frankreich, Österreich und den skandinavischen Ländern werden DRG zur Berechnung der Vergütung bestimmter Behandlungen verwendet. Allerdings betrifft diese Art der Vergütung dort nur einen geringen Anteil des Gesamterlöses der Krankenhäuser.

Als Vorbild des deutschen DRG-Systems diente das australische System (AR-DRG). Dieses System weist im internationalen Vergleich den höchsten Differenzierungsgrad bezüglich der jeweiligen Schwere des abgerechneten Krankheitsfalles auf. Es wurde deshalb als Vorlage für das deutsche DRG-System genutzt (Neubauer und Nowy 2001).

In Deutschland wurde im Jahr 2001 das „Institut für das Entgeltsystem im Krankenhaus (InEK)“ gegründet. Dieses Institut wurde von der Bundesregierung beauftragt, mit Hilfe des zugrunde gelegten australischen DRG-Katalogs einen spezifisch deutschen DRG-Katalog zu entwickeln. Dafür musste zunächst die Höhe der jeweiligen Fallpauschale bestimmt werden. Diese errechnet sich aus den Kosten, die durchschnittlich bei Patienten mit einer bestimmten Diagnose anfallen. Während der Konvergenzphase war das InEK für die jährliche Überarbeitung und Anpassung der AR-DRG an die in Deutschland tatsächlich anfallenden Kosten und bestehenden Bedingungen zuständig. Durch diese schrittweise Anpassung kann seit 2007 laut Deutscher Bundesregierung von einem eigenständigen deutschen Fallpauschalensystem gesprochen werden (Deutsche Bundesregierung 2007). Die AR-DRG sind in spezifisch deutsche G-DRG (German-DRG) umgewandelt worden, die nun als Grundlage für die Abrechnung der Krankenhäuser mit den Krankenkassen dienen. Das InEK hat auch nach Beendigung der Konvergenzphase die Aufgabe, jährlich einen neuen DRG-Katalog zu entwickeln, der den Ärzten und Kodierkräften als Kodiergrundlage dient. Durch den jährlich neu erscheinenden Katalog soll eine bessere Abbildung der tatsächlich anfallenden Kosten für die jeweilige Fallpauschale erreicht werden. 


\subsubsection{Berechnung des Fallerlöses eines Krankenhausaufenthalts}

Um den einzelnen Fallerlös eines Krankenhausaufenthalts, und damit den finanziellen Wert einer Fallpauschale zu errechnen, werden der Basisfallpreis und das Relativgewicht eines Falles benötigt. Das Relativgewicht ist ein vom InEK festgelegter Wert. Es wird eingeschätzt nach dem Aufwand und der Schwere der Leistung und gibt an, um welchen Wert die Behandlungskosten einer Fallpauschale vom Basisfallpreis abweichen. Ist der Basisfallpreis identisch mit den Behandlungskosten, so ist das Relativgewicht $=1$. Das Relativgewicht ist ein Erlösäquivalent, das auf Basis der Kostenkalkulation von dem InEK festgelegt wird.

Der Fallerlös einer stationären Behandlung errechnet sich aus den durchschnittlich anfallenden Fallkosten, dem sogenannten Basisfallpreis, multipliziert mit dem DRG-Relativgewicht. Der Basisfallpreis war zu Beginn der Konvergenzphase zunächst klinikintern festgelegt und wurde während dieser Phase in mehreren Schritten in einen Landesbasisfallpreis umgewandelt, welcher seit Januar 2009 in jedem deutschen Bundesland einheitlich ist..

\section{Fallerlös = Basisfallpreis $x$ Relativgewicht}

Jedem Patienten wird während seines stationären Aufenthalts eine sogenannte „Hauptdiagnose“ zugewiesen. Diese wird folgendermaßen definiert: Eine Hauptdiagnose ist „die Diagnose, die nach Analyse als diejenige festgestellt wurde, die hauptsächlich für die Veranlassung des stationären Krankenhausaufenthaltes des Patienten verantwortlich ist.“ (Deutsche Krankenhausgesellschaft 2001). Die Hauptdiagnose entscheidet darüber, mit welcher DRG ein Klinikaufenthalt kodiert wird. Da große individuelle Unterschiede in dem Behandlungsaufwand von Patienten mit gleicher Hauptdiagnose - und damit gleicher DRG - entstehen können, werden bei der Erlöserrechnung sowohl Komorbiditäten und Komplikationen als auch Nebendiagnosen und das Alter des Patienten mit berücksichtigt. Zur Veranschaulichung folgt nun ein Beispiel, das anhand der Kodierrichtlinien von 2007 errechnet wurde:

Ein Patient mit der Diagnose „Bronchialkarzinom“ wird während seines stationären Aufenthalts konservativ auf einer internistischen Station behandelt. Sein Klinikaufenthalt wird mit der Fallpauschale E71 kodiert. Treten während der stationären Behandlung Komplikationen auf oder bestehen bei dem Patienten schwere Komorbiditäten, so wird aus der Fallpauschale E71 die 
Fallpauschale E71a. Das Relativgewicht dieser DRG beträgt 1,275. Wird ein Basisfallpreis von $2900 €$ angenommen, ergibt sich ein Fallerlös von rund $3700 €$.

Die stationäre Behandlung eines Patienten mit derselben Diagnose, aber ohne Komorbiditäten oder Komplikationen, wird mit der Fallpauschale E71b kodiert. Das Relativgewicht dieser DRG beträgt 0,605. Bei gleichem Basisfallpreis von $2900 €$ errechnet sich ein Erlös von ca. $1750 €$.

Aus diesem Beispiel wird ersichtlich, wie wichtig eine korrekte Kodierung von Haupt- und Nebendiagnosen ist. Der Arzt, der das Kodieren vornimmt, ist für die korrekte Vergütung des jeweiligen Falls direkt mitverantwortlich. Dabei hat sich gezeigt, dass es je nach Kodierungsqualität Erlösunterschiede bis zu 20\% gibt (Neumann 2006). Pro Patient wird eine Kodierzeit von ca. 20 Minuten angenommen (Afflerbach 2002), so dass ein Mehraufwand an Dokumentationsarbeit zu erwarten ist.

\subsubsection{Berechnung des Klinikbudgets}

Zur Berechnung des Klinikbudgets wird die Summe der Relativgewichte, der sogenannte CaseMix-Index, verwendet. Er ist ein Indikator für die Kostenintensität und damit die „Schwere“ der Behandlungsfälle. Zur besseren Übersichtlichkeit sind die Begriffe in der folgenden Abbildung noch einmal kurz erläutert:

Basisfallpreis (BR - base rate) gab zunächst klinikintern, seit Ende der Konvergenzphase bundesland-einheitlich, die durchschnittlichen Fallkosten / den Fallwert an;

Relativgewicht (RG) gibt an, um wie viel teurer oder billiger die Behandlung eines Patienten gegenüber dem durchschnittlichen Behandlungsfall ist, und definiert so den Erlös für die einzelne Fallpauschale;

Case-Mix-Index (CMI) errechnet sich aus der Summe der Relativgewichte.

Abbildung 1.2.2. Erläuterungen zur Berechnung des Klinikbudgets 
Das individuelle Budget einer Klinik errechnet sich aus der Fallzahl multipliziert mit dem Basisfallpreis (BR) und dem Case-Mix-Index (CMI):

\section{Budget $=$ Fallzahl $\times$ BR $\times$ CMI}

Da sowohl der Case-Mix-Index als auch der Basisfallpreis nicht von den Kliniken beeinflusst werden können, ergibt sich, dass die Kliniken seit dem Ende der Konvergenzphase 2009 ihr Budget nur durch eine hohe Fallzahl steuern können. Für die Krankenhäuser gilt nun, dass sie gewinnbringend arbeiten, wenn die Kosten der Leistungserbringung unter der Höhe der Fallpauschale liegen. Die Politik erhofft sich dadurch eine effizientere Krankenversorgung und damit Kosteneinsparungen.

\subsection{Fallpauschalen als politisches Steuerungsinstrument}

Durch die Umstellung der Vergütung auf Fallpauschalen haben sich die Gesetzgeber mehr Qualität, Transparenz und Wirtschaftlichkeit im Gesundheitswesen (Bundesministerium für Gesundheit 2001) erhofft. Laut Fallpauschalengesetz werden nur noch solche Leistungen vergütet, die ordnungsgemäß dokumentiert bzw. durch das Klassifikationsschema des Fallpauschalenkatalogs abgedeckt werden (Deutscher Bundesrat 2002). Die Krankenhäuser sind seit der DRG-Einführung verpflichtet, regelmäßige Qualitätsberichte zu veröffentlichen, um ihre internen Ergebnisse der Öffentlichkeit zu präsentieren. Zu diesen Ergebnissen gehören z.B. die Fallzahlen einer Diagnose sowie die Anzahl und Art der durchgeführten Behandlungen. Dadurch soll der Öffentlichkeit ein Einblick in das Behandlungsspektrum der Kliniken gegeben werden.

Zur Sicherung und Verbesserung der Behandlungsqualität hat die Politik externe Überwachungsmechanismen geschaffen. So wurden z.B. Mindestanforderungen an Struktur- und Ergebnisqualität festgelegt. Die Gesetzgeber fordert seit der DRG-Einführung Mindestmengen für stationär durchgeführte Behandlungen (Deutscher Bundesrat 2002). Kliniken dürfen eine bestimmte Behandlung nur dann anbieten, wenn diese der Mindestmenge entsprechend häufig in der Klinik angewendet wird. Der Gesetzgeber stellt somit einen direkten Zusammenhang zwischen Quantität und Qualität her und hofft dadurch eine bessere Behandlungsqualität zu erreichen. 
Ein durch das DRG-System bedingter höherer Kostendruck auf die Kliniken soll zu einer effizienteren Nutzung der Ressourcen führen. Ärzte und Pflegekräfte werden dazu angehalten, Behandlungsabläufe sinnvoll und kostengünstig zu gestalten. Außerdem schafft die Einführung von Fallpauschalen Anreize, einen Klinikaufenthalt möglichst kurz zu halten. Mit Hilfe dieser Veränderungen erhofft sich die Bundesregierung, die zu erwartenden Gesundheitskosten einzudämmen.

In anderen Ländern gibt es bereits Studien über die Veränderungen durch die Einführung eines DRG-Abrechnungssystems. Auf diese wird im folgenden Abschnitt eingegangen.

\subsection{Erfahrungen mit DRG-Abrechnungssystemen in anderen Ländern}

Die Idee eines DRG-Systems stammt aus den USA. Es wurde in den 1970er Jahren von Wissenschaftlern an der Universität Yale erarbeitet, um die medizinische Versorgung zu objektivieren und so einen besseren Qualitätsstandard zu gewährleisten. Seit den 80er Jahren des letzten Jahrhunderts wird das DRG-System in den USA für die Patientengruppe der über 65Jährigen angewendet, die über das Medicaid-Programm versichert sind. Dieses Programm bietet Personen mit geringem Einkommen einen Krankenversicherungsschutz.

Auch in anderen Ländern (Australien, Frankreich, Kanada, Österreich, Skandinavien) wird mit DRG gearbeitet (Neumann 2006). Das deutsche System basiert, wie schon erwähnt, auf dem Australiens (AR-DRG). Es gibt aber bisher noch kein anderes Land, in dem alle Patientengruppen des stationären Sektors in das DRG-System eingebunden sind. Deutschland ist hier Vorreiter, weshalb kritische Stimmen von einem „Menschenversuch an 15 Millionen Fällen“ (Keil 2001, S.1) sprechen.

Die im Folgenden vorgestellten Studienergebnisse stammen aus den USA. Nur in diesem Land wurden, wie in Deutschland, die DRG flächendeckend und für alle Leistungen des stationären Sektors eingeführt. Aus diesem Grund erscheinen diese Studien besonders geeignet, um eventuelle Veränderungen in der Behandlung für Deutschland vorauszusagen (Sangha 1999). Im Unterschied zum deutschen System werden in den USA allerdings nur die über 65-jährigen Patienten im Medicaid-Programm berücksichtigt.

Die Studien aus den USA zeigen Veränderungen, die zum Teil auch auf die Behandlungssituation in Deutschland übertragen werden können. Die Erhebung der Studiendaten wurde sowohl 
retrospektiv durch Auswertung von Patientenakten als auch mit Hilfe direkter Datenerhebung durchgeführt. Verglichen wurden jeweils die Daten vor, während und nach der Einführung des DRG-Systems. Folgende Daten sind für eine ethische Beurteilung des DRG-Systems besonders interessant:

- durchschnittliche Verweildauer der Patienten im Krankenhaus,

- Anzahl der Überweisungen in stationäre Behandlung,

- Pflegebedarf bei Entlassung,

- Veränderungen der medizinischen Infrastruktur,

- Letalitätsraten,

- Behandlungsqualität und Zustand des Patienten bei Entlassung.

\subsubsection{Durchschnittliche Verweildauer von Patienten im Krankenhaus}

Aus folgenden gesichteten Studien (DesHarnais et al.1988, Fitzgerald et al. 1987, Gerety et al. 1989, Guterman et al. 1988, Kahn et al. 1990b, Kosecoff et al. 1990, Mayer-Oakes et al. 1988, Rogers et al. 1990, Rubenstein et al. 1990, Sager et al. 1987, Sager et al. 1989) geht hervor, dass die durchschnittliche Verweildauer im Krankenhaus nach der Einführung des DRG-Systems signifikant abnimmt.

Kahn et al. untersuchten dazu fünf Patientengruppen mit jeweils unterschiedlichen Erkrankungen (Herzinsuffizienz, Myokardinfarkt, Pneumonie, Apoplexie und Hüftfraktur). Die Abnahme der Verweildauer hing dabei von der jeweiligen Krankheit ab (Kahn et al. 1990b): Bei Herzinsuffizienz sank die Verweildauer der Patienten um durchschnittlich 21\%, bei Myokardinfarktpatienten um 18\%, bei Patienten mit Pneumonie um 14\%, bei Apoplexiepatienten um $32 \%$ und bei Patienten mit Hüftfrakturen um $28 \%$.

Daraus ergibt sich ein durchschnittlicher Rückgang der Verweildauer bei den genannten Krankheitsbildern um 24\%, was eine durchschnittliche Reduktion der Verweildauer von 14,4 auf 11 Tage bedeutet.

Eine Erklärungsmöglichkeit für dieses Studienergebnis ist, dass das DRG-System einen finanziellen Anreiz gibt, die Patienten nach möglichst kurzer Zeit zu entlassen, während es bei 
dem vorher angewendeten System der Abrechnung nach Tagessätzen vorteilhafter war, den Patienten länger im Krankenhaus zu behalten.

\subsubsection{Anzahl der Überweisungen in stationäre Behandlung}

Aus der Studie von DesHarnais et al. (1988) wird ersichtlich, dass die Anzahl der in ein Krankenhaus überwiesenen Patienten signifikant abnimmt. Auch makroökonomische Daten belegen diesen Trend. So sind in den USA im Zeitraum von 1982 bis 1985 die stationären Überweisungen um über 10\% zurückgegangen (Sangha 1999).

Mit diesem Ergebnis hatte man nicht gerechnet, denn für die Kliniken ist es profitabler, eine möglichst große Patientenzahl zu behandeln. Es hätte also dazu kommen können, dass die Kliniken versuchen, ihre Fallzahlen zu erhöhen, indem ein Patient möglichst schnell entlassen wird, um ihn dann relativ bald mit der gleichen Diagnose wieder zu behandeln. Sangha erklärt sich dieses Ergebnis damit, dass gleichzeitig mit den DRG Überwachungsmechanismen eingeführt wurden, die ein solches Vorgehen gar nicht erst ermöglichen. Die Stichhaltigkeit dieses Zusammenhangs lässt sich aus der Studie jedoch nicht ableiten.

\subsubsection{Pflegebedarf bei Entlassung}

Die Studien von DesHarnais et al. (1988), Guterman et al. (1988), Fitzgerald et al. (1987), Kahn et al. (1990b) und Kosecoff et al. (1990) kommen übereinstimmend zu dem Ergebnis, dass der Pflegebedarf nach Entlassung der Patienten signifikant zunimmt. Die Querschnittsstudie von Fitzgerald et al. in einem Universitätskrankenhaus in Indianapolis zeigt, dass der Prozentsatz an Patienten, die nach einer Hüftgelenksfraktur im Zeitraum von 1981 bis 1985 in Pflegeeinrichtungen entlassen wurden, von 21\% auf 48\% angestiegen ist. Ein Anstieg von 13\% auf 19\% ist bei Patienten zu verzeichnen, die nach ihrem Krankenhausaufenthalt auf häusliche Pflege angewiesen waren (Fitzgerald et al. 1987).

Die Studie von Kahn et al. kommt dagegen bei der Überprüfung von fünf Patientengruppen mit jeweils unterschiedlichen Erkrankungen (Herzinsuffizienz, Herzinfarkt, Lungenentzündung, Schlaganfall, Hüftfraktur) nur zu einem durchschnittlichen Anstieg der ins Pflegeheim entlassenen Patienten von 4\%. Außerdem wird in dieser Studie bestritten, dass eine signifikante 
Steigerung der Langzeitpflege festzustellen ist (Kahn et al. 1990b). Diese unterschiedlichen Ergebnisse sind möglicherweise durch differierende lokale Verhältnisse und Unterschiede in der Patientenpopulation zu erklären.

\subsubsection{Veränderung der medizinischen Infrastruktur}

In den Studien zur Veränderung der medizinischen Infrastruktur lässt sich eine signifikante Abnahme der vorgehaltenen Bettenzahl erkennen. Laut der Studie von Mayer-Oakes et al. 1988, in der drei Intensivstationen in Kalifornien untersucht wurden, kommt es zu einer Reduktion vorgehaltener Betten um 31\%. Die Bettenzahl wurde von 121 auf 83 reduziert. Auch die Rehabilitationsmaßnahmen bei Patienten mit Hüftfraktur gingen nach Einführung der DRG von durchschnittlich 9,7\% Physiotherapiesitzungen auf 4,9\% Sitzungen zurück.

\subsubsection{Letalitätsrate}

Eine Erhöhung der Letalitätsrate ist in keiner der gesichteten Studien nachweisbar. Bei einigen Krankheiten nahm die Letalitätsrate sogar ab, wogegen sie bei anderen anstieg. Daraus lässt sich schließen, dass die Sterberate in Korrelation zu der jeweiligen Krankheit zu sehen ist.

Kahn et al. stellen fest, dass die Letalitätsrate 180 Tage nach Einweisung in ein Krankenhaus bei den fünf untersuchten Patientengruppen mit den Diagnosen „Herzinsuffizienz“, „Schlaganfall“, „Hüftfraktur“, „Herzinfarkt“ und „Lungenembolie“ durchschnittlich von 29,6\% auf 29\% zurückging (Kahn et al. 1990a). Allerdings hing es von der jeweiligen Erkrankung ab, ob bzw. wie sehr die Letalitätsrate gesunken ist. So gab es eine leichte Erhöhung der Sterberate bei Patienten mit Herzinfarkt oder Lungenentzündung. Gleichzeitig nahm aber die Letalitätsrate im Krankenhaus insgesamt von $16,1 \%$ auf $12,8 \%$ ab. Dies lässt sich durch eine Verlegung des Sterbeortes erklären, die in den Studien von Sager et al. 1987 und 1989 nachgewiesen werden konnte. 


\subsubsection{Behandlungsqualität und Zustand der Patienten zum Zeitpunkt der Entlassung}

In der Studie von Kosecoff et al. (1990) wird belegt, dass sich der Gesundheitszustand von Patienten bei der Entlassung nach Einführung der DRG verschlechtert hat. Dabei werden drei Indikatoren gesundheitlicher Probleme unterschieden:

- „Instabilität bei Entlassung“, hierzu zählen die im Krankenhaus entstandenen Gesundheitsprobleme;

- „Krankheit (sickness) bei Entlassung “, also ob der Patient noch dieselben Beschwerden wie bei Einweisung in das Krankenhaus hat;

- „abnorme letzte Laborwerte“.

Immerhin rund $85 \%$ der entlassenen Patienten waren in einem stabilen Gesundheitszustand, allerdings stieg der Anteil der Patienten, die instabil entlassen wurden, nach Einführung der DRG signifikant an. Besonders gestiegen ist der Prozentsatz der Patienten, die mit einer oder mehrerer „Instabilitäten“ nach Hause entlassen wurden. Laut Kosecoff et al. (1990) kam es zu einem Anstieg von 15\% auf 18\%. Es besteht ein signifikanter Mangel an medizinischer Versorgung bei der Gruppe der nach Hause entlassenen Patienten und nicht bei denen, die in eine Pflegeinstitution entlassen wurden.

\subsubsection{Zusammenfassung der Studienergebnisse}

Einige Ergebnisse der gesichteten Studien besitzen für die deutsche Diskussion zum Thema DRG eine besondere Relevanz:

- die durchschnittliche Verweildauer der Patienten im Krankenhaus nimmt ab,

- der Pflegebedarf bei Entlassung steigt an,

- es kommt zur Abnahme der vorgehaltenen Bettenzahl,

- die Letalitätsrate erhöht sich nicht signifikant,

- die Patienten werden in einem instabileren Zustand nach Hause entlassen. 


\subsection{Gefahren der DRG-Einführung in Deutschland aus ethischer Perspektive}

Die Studien aus den USA lassen Rückschlüsse auf die Veränderungen durch die DRGEinführung in Deutschland zu. Gesundheitsexperten, Ethiker und Mediziner befürchten, dass die Einführung von Fallpauschalen sich nicht nur positiv auf die Versorgung kranker Patienten auswirkt. Es besteht Konsens, dass die sozialen und humanitären Aufgaben eines Krankenhauses nicht ökonomischen Erwägungen geopfert werden sollen (Fritze 2002).

Eine der größten Sorgen vor der DRG-Einführung war, dass es trotz der oben aufgeführten Ziele der Regierung zu einer sinkenden Behandlungsqualität in der Krankenversorgung komme. Diese Sorge liegt darin begründet, dass es für die Kliniken wirtschaftlich von Vorteil ist, Patienten möglichst schnell und nach einer möglichst günstigen Behandlung wieder zu entlassen. Diese Vermutung wird durch die Studienergebnisse aus den USA bestätigt (s. Kap. 1.4.1.). Von einigen Autoren wurde ein Rückgang der Behandlungsqualität befürchtet (Jonitz 2002, Keil 2001, Kölking 2001, Laschet 2001, Wehkamp 2002, Zaddach 2001). In der Medienlandschaft wurde der Begriff der „blutigen Entlassung“ geprägt. Dieser Begriff zeigt die Angst vor einer Klinikentlassung in noch nicht ausreichend gesundem Zustand und somit vor einer Minderversorgung von Patienten. Dem entgegenzusetzen ist allerdings, dass eine frühe Entlassung des Patienten nicht unbedingt im Gegensatz zu Patientenwohl und guter Behandlungsqualität steht. Wird ein Patient in stabilem Gesundheitszustand nach Hause entlassen und ist dessen ambulante Weiterbetreuung gesichert, so sei eine frühzeitige Entlassung nicht als negativ zu bewerten (Buhr 2006).

Durch das neue Abrechnungssystem wächst der Druck auf den behandelnden Arzt und die Kliniken, eine bestimmte Kostengrenze nicht zu überschreiten. Anderenfalls ergibt sich ein finanzieller Verlust für die Klinik. So kann es zu Interessenkonflikten und zu einer Einschränkung der ärztlichen Autonomie kommen (Kölking 2001, Leititis 2000, Wehkamp 2002, Zaddach 2001). Die Ärzte fürchten eine „Begrenzung ihrer Therapiefreiheit“ (Wehkamp 2002), da sie nun durch Kostengrenzen in ihren therapeutischen Möglichkeiten eingeschränkt werden könnten.

Durch den erhöhten Dokumentationsaufwand verändert sich außerdem der ärztliche Arbeitsbereich. Der direkte Kontakt mit dem Patienten rückt in den Hintergrund des ärztlichen 


\section{EINLEITUNG}

Arbeitsfeldes, denn der Dokumentation kommt eine besondere Bedeutung zu: Die Ärzte tragen nun eine direkte Verantwortung für die Einnahmen des Arbeitgebers, da nur solche Leistungen vergütet werden, die systemkonform dokumentiert wurden.

Eine weitere Folge der DRG-Einführung besteht in einer Veränderung der medizinischen Infrastruktur in Deutschland. Das Wirtschaftsberatungsunternehmen McKinsey sagt voraus, dass die Umstellung bis zu 400 deutsche Krankenhäuser überflüssig macht, da durch ein Absinken der Verweildauer ein Rückgang der Belegungsquote zu erwarten sei (Hehner et al. 2002). Die Studie von Hehner prognostiziert außerdem vermehrt Krankenhausfusionen, da besonders kleinere Kliniken durch den hohen Kostendruck nicht alleine existieren können.

Um die Behandlungsqualität $\mathrm{zu}$ sichern, fordern die Gesetzgeber Mindestmengen von Behandlungen (Deutscher Bundesrat 2002). Diesem Vorhaben wird von ärztlicher Seite entgegengesetzt, dass „die Qualitätssicherung bei Fallpauschalen [...] ein Vehikel ist, um über die Ermittlung und Vorgabe von Mindestzahlen das Leistungsangebot einzelner Häuser zu steuern“ (Sitzler 2001, S.51). Kleinere und ländliche Kliniken werden die geforderten Mindestmengen nicht für alle bislang angebotenen Behandlungen erreichen können und deshalb bestimmte Leistungen nicht mehr durchführen dürfen. Dadurch kommt es zu einer politischen Steuerung des Leistungsangebots der Krankenhäuser. Außerdem wird es zu einer Umgestaltung der deutschen Kliniklandschaft von vielen kleinen in ländlichen Gebieten gelegenen Kliniken zu spezialisierteren Zentren kommen (Lenk et al. 2005). Diese Zentren bieten dann ein kleineres Spektrum von Behandlungen an. Im Gegenzug werden die angebotenen Behandlungen aber häufiger durchgeführt. Die Umstellung auf spezialisierte Kliniken hat zur Folge, dass sich die bisherige Krankenhausstruktur in Deutschland verändern wird. In den letzten Jahrzehnten hat sich in Deutschland eine hohe Dichte an Krankenhäusern entwickelt, so dass die Versorgung auch in ländlichen Regionen gesichert war. Durch die Mindestmengenregelung könnte es dazu kommen, dass sich die bislang gute medizinische Infrastruktur besonders in ländlichen Regionen verschlechtert. Die Kliniken müssen sich, um die Mindestmengenanforderung zu erfüllen, auf bestimmte Behandlungen spezialisieren. Gelingt eine solche Spezialisierung nicht, könnte es zur Schließung dieser Kliniken kommen. Dies gefährdet die flächendeckende Grundversorgung besonders in ländlichen Regionen. 
Nach Angaben des Statistischen Bundesamtes ist die Zahl der Kliniken von 2411 im Jahr 1991 auf 2087 im Jahr 2007 gesunken. Dabei hat sich auch die Zahl der vorgehaltenen Betten von 665.565 auf 507.000 im selben Zeitraum vermindert. Das entspricht fast 160.000 Betten. Das Phänomen der Schließung von Kliniken und der Bettenreduktion besteht nicht erst seit der DRGEinführung, denn bereits im Jahr 2003 gab es in Deutschland nur noch 2197 Krankenhäuser mit 541.901 Betten. Es ist zu erwarten, dass dieser Trend in den nächsten Jahren weiter fortschreiten wird.

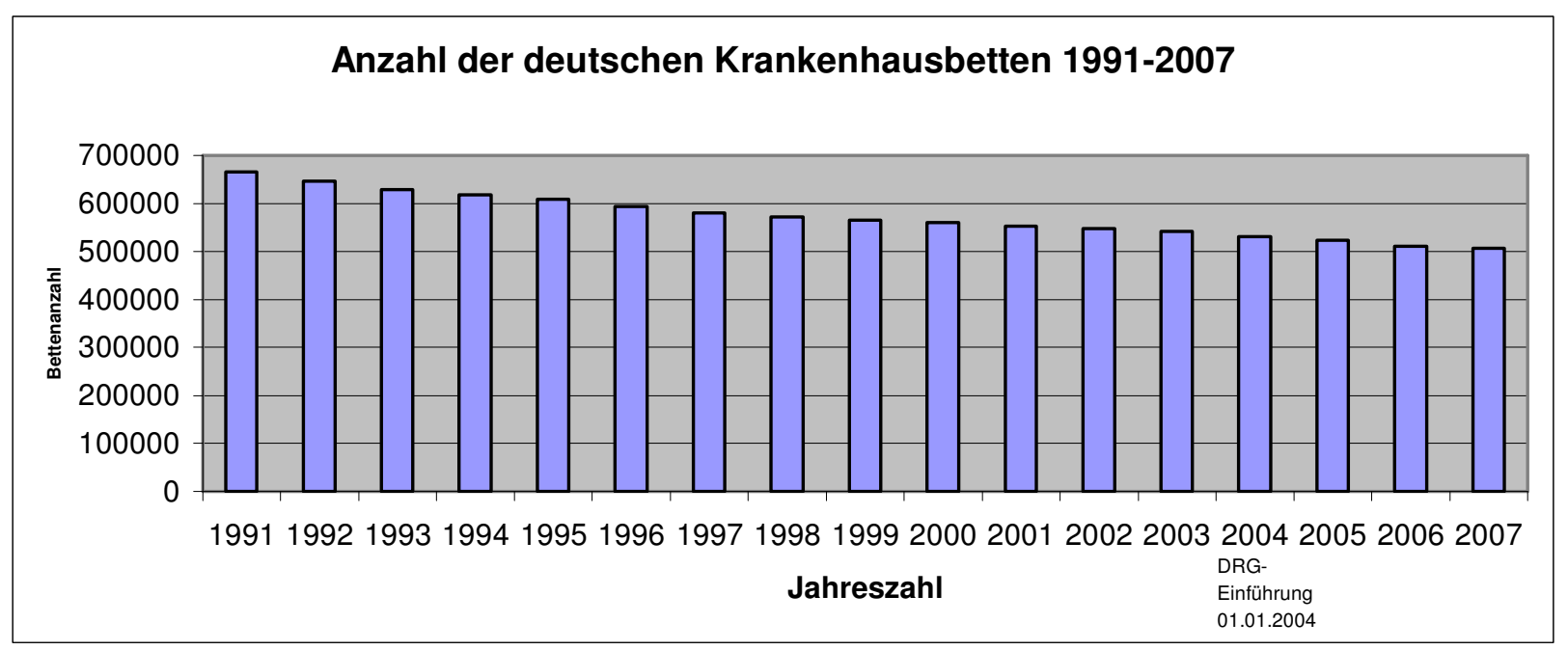

Abbildung 1.5. Anzahl der vorgehaltenen Betten in deutschen Kliniken im Vergleich 1991 bis 2007 (Quelle: Statistisches Bundesamt 2008)

Der Abbau von Betten sei ein von der Regierung beabsichtigter Nebeneffekt der DRGEinführung (Fritze 2002). Dies wird durch die Aussage der Bundesregierung bestätigt, dass trotz der bereits vorgenommenen Bettenreduktion auch im Jahr 2007 im internationalen Vergleich Überkapazitäten in der stationären Krankenversorgung bestünden (Deutsche Bundesregierung 2007).

Neben den bereits aufgeführten Veränderungen werden Einschnitte in der Intensivmedizin und für Kliniken der Maximalversorgung befürchtet (Sitzler 2001). Laut Sitzler tragen ,insbesondere Kliniken der Maximalversorgung [...] das erhebliche Risiko, eine Unterbewertung von Fällen mit hohem Aufwand selbst tragen zu müssen. Wegen des hohen Anteils dieser Fälle am Gesamterlös im DRG-System bestehen für Maximalversorger wesentlich höhere betriebswirtschaftliche 


\section{EINLEITUNG}

Risiken als für Häuser geringerer Versorgungsstufen“ (ebd., S. 53). Auch Leititis weist auf die Risiken für die Krankenhäuser der Maximalversorgung hin (Leititis 2000).

Neubauer befürchtet Konsequenzen für Patienten, die zu früh in den Rehabilitations-Bereich entlassenen werden. Die Prognose für eine erfolgreiche Wiederherstellung der Betroffenen könnte dadurch signifikant schlechter werden (Neubauer et al. 2001). Die Sozialdienste erwarten ihrerseits eine Überlastung nach DRG-Einführung und kritisieren, dass die Kosten für zusätzliche anfallende komplementäre Hilfsmittel im Rahmen des Fallpauschalengesetzes nicht berücksichtigt werden (Beyer-Rehfeld 2001). Ein Wandel in der medizinischen Infrastruktur wird sich nicht nur im stationären Bereich, sondern auch in der ambulanten Versorgung widerspiegeln. Durch die Abnahme der Verweildauer im Krankenhaus werden Patienten häufiger ambulante medizinische Betreuung in Anspruch nehmen und es wird zur Zunahme von Einweisungen in Pflegeheime kommen (Neumann 2006).

In der Debatte um die Einführung von Fallpauschalen wird außerdem diskutiert, ob das neue System für bestimmte Patientengruppen, vor allem für chronisch Kranke, Schwerkranke oder Sterbende geeignet ist. Die Versorgung von Schwer- bzw. chronisch Kranken wird durch die Anreize des DRG-Systems unter ökonomischen Gesichtspunkten für Kliniken „unattraktiv“. Die oben genannten Patienten brauchen eine intensive Pflege und zusätzlich vermehrt psychische Unterstützung. Diese Aspekte lassen sich durch DRG nur schwer abbilden bzw. werden gar nicht berücksichtigt und somit auch nicht vergütet. Beneker schreibt in seinem Artikel „Krankt die stationäre Palliativversorgung am Fallpauschalensystem?“, dass die palliativmedizinische Begleitung schwerstkranker Menschen kaum pauschalisierbar ist (Beneker 2006).

Chronisch kranke Patienten, denen eine Fallpauschale zugeordnet wird, können in unterschiedlichem Maße von anderen Krankheiten, sogenannten Komorbiditäten, betroffen sein. Die Behandlung eines Patienten mit mehreren Komorbiditäten ist aufwendiger und teurer als die von Patienten ohne Komorbiditäten. Innerhalb des DRG-Systems besteht die Möglichkeit, Komorbiditäten als Nebendiagnosen zu berücksichtigen, allerdings beruht die Fallpauschale vor allem auf der jeweiligen Hauptdiagnose. Ob das Kodieren von Nebendiagnosen ausreicht, um die anfallenden Mehrkosten zu decken und chronisch Kranke ihrem Gesundheitszustand gemäß zu behandeln, ist nicht sicher. Durch die Einführung eines „lernenden Systems“ besteht nach Angaben der Bundesregierung aber die Möglichkeit, die Erlöse an die gegebenen Bedingungen anzupassen. Aus diesem Grund bringt das Institut für das Entgeltsystem im Krankenhaus (InEK) 
wie oben bereits erwähnt jährlich einen neuen Kodierkatalog heraus, der die Abbildung von Krankheiten im DRG-System verbessern soll.

Wenn die jeweilige Pauschale die anfallenden Behandlungskosten nicht deckt, könnten „Kliniken die Aufnahme von multimorbiden oder behinderten Patienten unter Kostengesichtspunkten ablehnen oder verzögern [...] und Patienten ohne Sicherung der erforderlichen bedarfsgerechten Anschlussbehandlung, Rehabilitation oder Nachsorge Möglichkeiten entlassen [werden]“ (DVfR 2001, S. 1).

Auch bei schwerkranken Patienten, die mit innovativen und kostenintensiven Therapien behandelt werden oder psychologische Betreuung benötigen, wie z.B. onkologische Patienten, bestehen diese Sorgen (Laschet 2001, Fachgruppe Klinische PsychologInnen 2001).

In seinem Gutachten für die Deutsche Krankenhausgesellschaft (DKG) 2006 kommt der Gesundheitsökonom Roeder aber zu dem Ergebnis, dass „für den großen Teil der Patientinnen und Patienten, die eine Standardversorgung benötigen, keine Versorgungsprobleme zu befürchten [sind]“ (Roeder 2006, S. 3).

\subsubsection{Onkologische Patienten und ihre ethische Relevanz}

Die vorliegende Studie untersucht die Konsequenzen der Einführung von Fallpauschalen am Beispiel von Patienten mit Bronchialkarzinom. Diese Patienten sind zu einem großen Teil schwer krank. Ihre Betreuung bedarf eines optimalen Zusammenspiels von Pflege, Psychologen, Palliativmedizinern und Onkologen. Da Fallpauschalen die technisch-apparative Medizin bevorzugen, und die zwischenmenschlichen Anteile der Behandlung unterrepräsentiert sind (Neumann 2006), stellt sich die Frage, ob es zu einer Unterversorgung von Schwerkranken und Sterbenden kommt. Die stationäre Versorgung dieser Patientengruppe basiert nicht nur auf der medizinisch-fachlichen Therapie, sondern auch auf menschlicher Zuwendung und einem adäquaten Umgang mit Krankheit und Tod. Für eine adäquate Behandlung benötigen die Pflegekräfte und Ärzte Zeit für Gespräche mit dem Patienten. Unter Berücksichtigung dieser Aspekte ist deshalb folgende Frage von besonderer Bedeutung: Kann individuelle Betreuung und menschliche Zuwendung gemäß der DRG-Richtlinien gewährleistet werden?

Durch eine fallpauschalisierte Abrechnung für Patienten mit Bronchialkarzinom entsteht ein weiteres Problem: In der DRG-Klassifikation werden neben der Hauptdiagnose besonders das 
Alter und die Komorbiditäten berücksichtigt. Im onkologischen Bereich werden jüngere Patienten häufig mit teureren und aggressiveren Methoden behandelt. Die Therapiekosten können bei gleicher Hauptdiagnose erheblich voneinander abweichen. Da die Auswahl der entsprechenden Medikamente oftmals der entscheidende ökonomische Faktor ist (Gröticke und Rasche 2001), stellt sich für die onkologischen Patienten ebenfalls die Frage, ob diese Patientengruppe im DRG-System korrekt abgebildet wird und die Patienten eine optimale Behandlung bekommen.

Eine Begleitforschung, die die Versorgung bzw. Versorgungsqualität von onkologischen Patienten abbildet, ist wichtig. Sie kann frühzeitig Probleme und Mängel aufdecken und Vorschläge für Verbesserungen machen.

\subsubsection{Ethische Implikationen}

Die gesichteten Studien aus den USA lassen Rückschlüsse auf in Deutschland zu erwartende Veränderungen und Konsequenzen für das Gesundheitswesen zu. Ziel der vorliegenden Studie ist es, Veränderungen sichtbar zu machen und sie unter ethischen Aspekten zu betrachten und zu bewerten. Dabei werden die möglichen Veränderungen am Beispiel von Patienten mit Bronchialkarzinom und ihren betreuenden Ärzten und Pflegekräften untersucht.

Die ethische Bewertung der Ergebnisse basiert auf der Prinzipienethik von Beauchamp und Childress (Beauchamp, Childress 2001). Diese amerikanischen Bioethiker stellen in ihrem Buch „Principles of biomedical ethics“ Prinzipien mittlerer Reichweite auf, die sich auf allgemein in der modernen Medizin akzeptierte Werte beziehen. Sie spiegeln die moralischen Grundlagen des ärztlichen Handelns wider. Beauchamp und Childress gehen dabei von vier gleichberechtigten Prinzipien aus:

- Autonomie

- Fürsorgeprinzip

- Nichtschädigungsprinzip

- Gerechtigkeit. 
Für die Analyse der Veränderungen während der Einführung von Fallpauschalen sind das Nichtschädigungsprinzip und das Prinzip der Gerechtigkeit von besonderer Relevanz, weil durch das DRG-System Konflikte zwischen dem heilberuflichen Ethos des Nichtschädigens bzw. der Gerechtigkeit und betriebswirtschaftlichen Interessen entstehen können (Wehkamp 2002).

Die DRG-Einführung wurde vor allem unter dem Aspekt der Behandlungsqualität, der gerechten Verteilung und der Benachteiligung von Patienten diskutiert. Würden die in den vorherigen Abschnitten beschriebenen Befürchtungen eintreffen, könnten das Prinzip des Nichtschädigens und das der Gerechtigkeit missachtet werden. Die beiden Prinzipien werden im Folgenden vorgestellt und in ihrer Bedeutung für die DRG-Einführung diskutiert.

\subsubsection{Nichtschädigungsprinzip}

Dem Prinzip des Nichtschadens liegt die Maxime des „Primum nihil nocere“ - „Vor allem füge niemandem Schaden zu!" - zugrunde (Beauchamp und Childress 2001, S. 113). Die Bedeutung dieser Maxime wurde schon in der Antike erkannt. Ärzte müssen alles, was dem Patienten Schaden zufügen kann, zu vermeiden suchen. Allerdings muss es dabei immer zu einem Abwägen zwischen Nutzen und Schaden kommen, denn wenn eine Behandlung zur Verbesserung des Gesundheitszustands führen soll, kann bzw. muss dafür unter Umständen ein durch die Behandlung entstandener Schaden in Kauf genommen werden.

Der Arzt darf seine medizinischen Fähigkeiten aber vor allem nicht dazu missbrauchen, einem anderen Schaden an Leib, Leben oder Eigentum zuzufügen. Vom Prinzip des Nichtschädigens grenzen Beauchamp und Childress das Wohltunsprinzip ab. Nach diesem Prinzip hat ein Arzt die Verpflichtung, seinem Patienten Gutes zu tun:

- Übel und Schaden soll vorgebeugt werden;

- $\quad$ Übel und Schaden sollen beseitigt werden;

- das Wohl soll praktiziert und gefördert werden.

Im Unterschied zum Prinzip des Nichtschädigens wird der Arzt beim Prinzip des Wohltuns verpflichtet, aktiv etwas zu tun, während das Nichtschädigungsprinzip vor allem fordert, 
Handlungen zu unterlassen, die dem Patenten Schaden zufügen. Beauchamp und Childress gehen davon aus, dass es Regeln gibt, die das Nichtschädigungsprinzip unterstützen (ebd., S. 117):

- töte nicht;

- verursache keine Schmerzen oder kein Leid;

- setze Niemanden außer Gefecht;

- verursache keine Verletzung;

- entziehe Niemandem die Lebensgrundlagen.

Diese Regeln müssen auch innerhalb des DRG-Systems gelten, ansonsten würde es zu einem Konflikt des ärztlichen Handelns mit dem Nichtschädigungsprinzip kommen.

Allerdings besteht im ärztlichen Alltag ständig ein Konflikt zwischen dem Prinzip des Wohltuns und des Nichtschädigens, denn der Arzt fügt, z.B. indem er einem Patienten Blut abnimmt, Schaden zu, um ihm so adäquat helfen zu können. Besonders in der Onkologie sind viele Therapiemöglichkeiten mit Nebenwirkungen behaftet, die den Patienten schädigen. Durch eine Chemotherapie wird z.B. einerseits der Versuch unternommen, einen Tumor zu verkleinern, andererseits wird aber in Kauf genommen, dass die Immunabwehr des Patienten geschwächt wird, ihm übel wird oder er an Haarausfall leidet. $\mathrm{Ob}$ ein Verstoß gegen das Nichtschädigungsprinzip gerechtfertigt ist, muss der Arzt zusammen mit seinem Patienten klären. So muss er ihn z. B. vor einer Behandlung über die Wirkungsweise und Nebenwirkungen von Medikamenten aufklären. Der Patient muss dann im Sinne der informierten Einwilligung (informed consent) der Therapie zustimmen.

In der vorliegenden Studie werden die Veränderungen, die durch eine fallpauschalisierte Abrechnung entstehen können, unter dem Aspekt der Einhaltung des Nichtschädigungsprinzips untersucht. Die Qualität der stationären Behandlung wird als Messkriterium dieser Untersuchung herangezogen. Sollte es durch das DRG-System $\mathrm{zu}$ einer Verschlechterung der Behandlungsqualität kommen, so wäre dies im Sinne der bereits beschriebenen Regeln ein Verstoß gegen das Prinzip des Nichtschädigens, denn dem Patienten wird Schaden - im Sinne einer Qualitätsverschlechterung - zugefügt. 
Für die vorliegende Studie wurden Patienten mit einem Bronchialkarzinom ausgewählt, die sich aus den oben genannten Gründen (s. Kap. 1.5.1.) besonders gut für eine Untersuchung der Veränderungen während der DRG-Einführung eignen. Um die Qualität der Behandlung möglichst umfassend zu untersuchen, nehmen an der vorliegenden Studie nicht nur Patienten, sondern auch Ärzte und Pflegekräfte teil.

Zunächst wird untersucht, ob und wenn ja wie sich die Qualität der stationären Behandlung verändert. Folgende forschungsleitende Fragen werden anhand dieser Studie untersucht:

- Gibt es aus Sicht der Studienteilnehmer eine Veränderung in der Behandlungsqualität von Bronchialkarzinompatienten während der DRG-Einführung?

- Verändert sich die Lebensqualität der Patientenpopulation während der Einführung von Fallpauschalen?

- Verändert sich der Pflegebedarf der Patienten bei Entlassung?

- Verändert sich die stationäre Letalitätsrate der Bronchialkarzinompatienten während der Einführung des DRG-Systems?

- Kommt es aus Sicht der Studienteilnehmer zu Einschränkungen in der Therapie- und Behandlungsfreiheit von Bronchialkarzinompatienten?

Leitende Aspekte für diese Fragestellungen sind folgende Überlegungen:

Eine Beurteilung der Behandlungsqualität kann indirekt durch die Bewertung der Lebensqualität und des Pflegebedarfs des Patienten bei Entlassung erfolgen. Wenn Patienten z.B. in einem schlechten Gesundheitszustand mit Schmerzen nach Hause entlassen werden, ohne dass die benötigte ambulante Betreuung gesichert ist, kann dies der ärztlichen Verpflichtung des Nichtschadens widersprechen, denn ein Arzt soll - laut den oben aufgestellten Regeln niemandem Schmerzen oder Leid zufügen.

Beauchamp und Childress gehen außerdem davon aus, dass das Nichtschädigungsprinzip sich nicht nur auf die Verpflichtung der Schadensvermeidung, sondern auch auf die Verpflichtung der Risikovermeidung bezieht (Beauchamp und Childress 2001, S. 117). Aus diesem Grund ist es interessant $\mathrm{zu}$ untersuchen, ob es $\mathrm{zu}$ einer Einschränkung der Therapieoptionen bei 
Bronchialkarzinompatienten kommt. Wird den Patienten eine Behandlung verwehrt oder werden sie nicht ihrer individuellen Situation entsprechend behandelt, so vergrößert sich das Risiko, dass diesen Patienten Leid oder Schaden zugefügt werden. Durch eine pauschale Abrechnung könnte es schwieriger werden, auf die individuellen Wünsche und Bedürfnisse von Patienten einzugehen.

Die Untersuchung der Entwicklung von Letalitätsraten ist wichtig, um zu erforschen, ob die Patienten durch die Einführung von Fallpauschalen ein höheres Risiko haben, an ihrer Erkrankung zu versterben. Dies wäre der größte „Schaden“, der den Patienten zugefügt werden könnte.

\subsubsection{Gerechtigkeitsprinzip}

Neben dem Prinzip des Nichtschädigens wird die Einführung von Fallpauschalen in der vorliegenden Studie auch unter dem Prinzip der Gerechtigkeit untersucht. Die Gerechtigkeit wird, wie oben bereits erwähnt, in der Prinzipienethik als eines von vier gleichwertigen Prinzipien angesehen. Der folgende Absatz gibt einen Überblick über das Prinzip der Gerechtigkeit aus der Sicht von Beauchamp und Childress.

Eine Debatte über die gerechte Verteilung von Gesundheitsleistungen (Allokation) ist laut Beauchamp und Childress deshalb notwendig, weil die Menschen einerseits einen ungleichen Zugang zu Gesundheitsgütern haben und andererseits die Kosten im Gesundheitswesen stark ansteigen (Beauchamp und Childress 2001, S. 225). Die beiden Autoren gehen davon aus, dass eine ,gerechte“ Verteilung fair, vergleichbar und angemessen für die jeweilige Situation einer Person sein soll (ebd., S. 226).

Sie unterscheiden zwei Arten von Gerechtigkeit:

- Formale Gerechtigkeit: „, Gleiche sollen gleich behandelt werden, Ungleiche sollen ungleich behandelt werden “( $e b d .$, S. 227);

- materielle Gerechtigkeit:

- ,Güter sollen je nach Bedarf verteilt werden“;

- , jeder Person den gleichen Anteil “;

- ,jeder Person entsprechend ihrer Leistung “; 


\section{EINLEITUNG}

- ,jeder Person entsprechend ihres Beitrags “;

- ,jeder Person entsprechend ihres Verdienstes “;(ebd., S. 228).

Die materielle Gerechtigkeit präzisiert in diesem Fall die Forderung nach formaler Gerechtigkeit. Eine Gesellschaft muss überlegen, welche Form der materiellen Gerechtigkeit den Rahmen der Gesellschaftsordnung bilden soll.

Damit von einem gerechten System gesprochen werden kann, muss neben der Verteilungsgerechtigkeit auch Chancengleichheit gewährleistet sein. Das bedeutet, dass die Patienten unabhängig von ihrem Wohnort, Bildungsstand, finanziellen Möglichkeiten etc. gleiche Chancen auf eine angemessene Behandlung erhalten.

Durch die Knappheit von Ressourcen entstehen jedoch Verteilungsprobleme. So kann nicht jede medizinische und technische Behandlung durchgeführt werden.

Um diese Probleme zu lösen, bedarf es zunächst eines effizienten Gesundheitssystems. In dem System müssen die Kosten so gering wie möglich gehalten werden, um eine sinnvolle Verteilung der knappen Ressourcen zu gewährleisten. Eine gerechte Verteilung ist nur möglich, wenn den beteiligten Personen eine faire Chance auf medizinische Güter gewährt wird. In einem gerechten System darf im Sinne der Chancengleichheit niemand aufgrund seiner Herkunft, des Geschlechts oder sonstiger äußerer Merkmale oder Einstellungen diskriminiert werden (ebd., S. 233-234). Diese Chancengleichheit ist nur gegeben, wenn die finanziellen Risiken von den Versicherten gemeinsam getragen und geteilt werden. In Deutschland besteht im Rahmen der gesetzlichen Krankenversicherungen ein solches Solidarsystem. Dieses wird durch die Öffentlichkeit finanziert und muss mit den vorhandenen Ressourcen umsichtig umgehen. Um eine gerechte Gesundheitsversorgung zu gewährleisten, muss innerhalb eines Gesundheitssystems geklärt werden, welche Bereiche der Versorgung durch die vorhandenen Einnahmen solidarisch finanziert werden sollen. Danach müssen die Ressourcen in diesen Bereichen „gerecht“ verteilt werden.

Zur Klärung des Gerechtigkeitsbegriffs wird die Definition von formaler und materieller Gerechtigkeit (s. o.) herangezogen: Eine gerechte Verteilung entsteht dann, wenn die Güter gleich, bedarfsgerecht und mit Chancengleichheit verteilt werden. Dabei ist, wie bereits erläutert, die Begrenztheit der Ressourcen zu berücksichtigen, da die vorhandenen Güter nicht ausreichen, um alle denkbar möglichen Behandlungen im medizinischen Bereich zu finanzieren. Daraus 
ergibt sich folgende Frage: Kann es durch die Einführung von Fallpauschalen im Sinne des oben genannten Gerechtigkeitsbegriffs zu einer chancengleichen und bedarfsgerechten medizinischen Versorgung kommen?

Die Verteilung der Ressourcen (Allokation) findet auf zwei Ebenen, der Mikro- und der Makroebene, statt (Marckmann 2004). Auf der Makroallokationsebene werden die vorhandenen Ressourcen auf die einzelnen Bereiche der medizinischen Versorgung aufgeteilt (Kliniken, ambulanter Sektor, medizinische Forschung etc.). Die Mikroallokationsebene beinhaltet die Verteilung der Ressourcen für einen konkreten Patienten. Auf dieser Ebene werden Probleme und eine ungerechte Behandlung von schwerkranken und multimorbiden Patienten befürchtet (DVfR 2001, Laschet 2001).

Ein durch die DRG-Einführung bedingtes Problem ist die Pauschalisierung von stationären Behandlungen. Durch eine pauschale Bezahlung kann es zu einer ungerechten Behandlung von schwerkranken und sterbenden Patienten kommen, denn diese Menschen benötigen eine besonders intensive pflegerische und ärztliche Betreuung, die über die technisch-apparative Medizin hinausgeht. Diese Betreuung könnte in einem System, in dem pauschale Beträge ohne Berücksichtigung des individuellen Pflegeaufwands gezahlt werden, gefährdet oder nicht mehr möglich sein. Aus diesem Grund ergeben sich für die vorliegende Studie folgende Fragestellungen:

\section{- Können Fallpauschalen individuell auftretende Probleme erfassen, und kann ein Patient seinem psychischen und physischen Gesundheitszustand entsprechend behandelt werden?}

Auf der Ebene der Makroallokation werden ebenfalls Veränderungen durch das DRG-System vorhergesagt (s. Kap. 1.5.). So wird die Reduzierung von Betten und das Zusammenlegung von Kliniken befürchtet (Lenk et al. 2005). Im Sinne des Gerechtigkeitsprinzips könnte es dadurch zu einer Benachteiligung der ländlichen Bevölkerung kommen, da diese Patienten einen weiteren Weg zum Behandlungsort zurücklegen müssen und ihnen dadurch gesundheitlicher und finanzieller Schaden entsteht. Damit wäre keine faire und bedarfsgerechte Behandlung gegeben, da es durch den Wohnort der Patienten zu einer Benachteiligung kommt. Die vorliegende Studie befasst sich deshalb auch mit folgender Frage: 
- Haben Patienten, die in ländlichen Regionen wohnen, einen fairen Zugang zu medizinischer Versorgung?

\subsubsection{Begründung der empirischen Untersuchung}

Die empirische Untersuchung soll zunächst die Beurteilung der Ausgangssituation vor Einführung der DRG erlauben. Außerdem wird in dieser Studie untersucht, wie sich die Bedingungen für die Behandlung von Patienten mit einem Bronchialkarzinom im Raum Südniedersachsen während der DRG-Einführung verändern. Um ein möglichst umfassendes Bild dieser Veränderungen zu erhalten, werden sowohl Patienten als auch die an ihrer Behandlung beteiligten Ärzte und Pflegekräfte befragt. Daneben werden die Daten der Stabsstelle Controlling des Universitätsklinikums Göttingen hinzugezogen, um mögliche Veränderungen während der DRG-Einführung sichtbar zu machen. Diese empirischen Untersuchungsergebnisse werden einer ethischen Analyse unterzogen. Dabei ist es wichtig zu berücksichtigen, dass aus der Beschreibung des Ist-Zustands nicht auf ein „Sollen“ geschlossen werden kann. Deshalb werden Ausgangszustand und Veränderungen während und nach der DRG-Einführung unter Berücksichtigung des Nichtschädigungsprinzips und des Prinzips der Gerechtigkeit untersucht. Erst durch eine Analyse, ob der Ausgangszustand bzw. der Folgezustand diesen Prinzipien standhalten, kann eine normative Bewertung erreicht werden. 


\section{MATERIAL UND METHODEN}

\subsection{Allgemeines}

Zur Beantwortung der in der Einleitung hergeleiteten Fragen wurden folgende empirische Untersuchungen durchgeführt:

- retrospektive Befragung von Patienten mit Bronchialkarzinom in drei aufeinander folgenden Jahren (2004-2006) mittels Fragebogen;

- persönliche qualitative Interviews mit Ärzten anhand eines Fragenkatalogs;

- persönliche qualitative Interviews mit Pflegekräften anhand eines Fragenkatalogs;

- Analyse von Daten aus dem Controlling des Universitätsklinikums Göttingen.

Die empirische Untersuchung zielt nicht nur auf die Darstellung von möglichen Veränderungen durch die Patienten ab, sondern bezieht auch die an der Behandlung beteiligten Personengruppen (Ärzte und Pflegekräfte) mit ein. Außerdem werden die Daten der Stabsstelle Controlling des Universitätsklinikums hinzugezogen, um auch hier mögliche Veränderungen während der DRGEinführung sichtbar zu machen.

\subsection{Durchführung der Patientenbefragung}

Vom Institut für Sozialmedizin, Epidemiologie und Gesundheitsökonomie der Universität Berlin wurde in Zusammenarbeit mit dem Institut für Ethik und Geschichte der Medizin und der Abteilung Hämatologie und Onkologie der Universität Göttingen ein standardisierter Fragebogen für Patienten mit Bronchialkarzinom zum Thema „Medizinethische und gesundheitsökonomische Aspekte der fallpauschalisierten Vergütung“ konzipiert. Mit Hilfe dieses Fragebogens wurden Patienten mit einem Bronchialkarzinom zu ihrem Klinikaufenthalt im jeweils vorangegangenen Jahr und ihrem Gesundheitszustand zum Zeitpunkt der Erhebung befragt. Die Studie wurde von der Ethikkommission der Universitätsmedizin Göttingen genehmigt. 
Durch die schriftliche Befragung konnte eine große Anzahl von Patienten erreicht werden. Diese Art der empirischen Untersuchung hat gegenüber persönlichen Interviews folgende Vorteile und wurde aus diesen Gründen ausgewählt:

- Interviewfehler werden vermieden;

- Antworten sind ,ehrlicher“ als bei Anwesenheit eines Interviewers;

- Antworten sind „überlegter“, da mehr Zeit zum Ausfüllen des Fragebogens gegeben ist;

- der Beantwortungszeitpunkt kann selber gewählt werden;

- die Zusicherung von Anonymität ist glaubwürdiger (Schnell et al.1999).

Mit Hilfe des Fragebogens haben die Patienten aus ihrer Sicht die eigene stationäre Behandlung im jeweils vorangegangenen Jahr beurteilt. Um eventuelle Veränderungen vor und während der Einführung des DRG-Systems sichtbar zu machen, wurden die Fragebögen in den Jahren 20042006 versandt. Die Patienten, die im ersten Jahr einen Fragebogen erhielten, waren im vorangegangenen Jahr (2003) in stationärer Behandlung und bewerteten die eigene stationäre Versorgung vor einer flächendeckenden Einführung von Fallpauschalen. Die beiden folgenden Befragungen wurden während der DRG-Einführung in den beiden darauffolgenden Jahren durchgeführt.

Im Fragebogen wurden besonders die individuelle Krankenversorgung und die persönliche Situation der Patienten berücksichtigt. In geschlossenen und offenen Fragen gaben die Patienten an, welche Art der Behandlung sie erhielten, wie zufrieden sie mit der Behandlung und der Betreuung durch Ärzte und Pflegekräfte waren und welche Sorgen und Ängste bezogen auf ihre Erkrankung sie hatten. Der letzte Teil des Fragebogens bezog sich auf den Gesundheitszustand des jeweiligen Patienten zum Zeitpunkt der Befragung.

In den Jahren 2004-2006 wurde der Fragebogen an Patienten im Raum Südniedersachsen verschickt, die auf Grund der Diagnose „Bronchialkarzinom“ in stationärer Behandlung waren. Dabei wurden Patienten berücksichtigt, die in den auf dieses Krankheitsbild spezialisierten Krankenhäusern im Raum Südniedersachsen in Behandlung waren. Damit sollte sichergestellt werden, dass möglichst alle stationär behandelten Patienten dieser Region mit der Diagnose 
„Bronchialkarzinom“ befragt werden konnten. Aus folgenden Kliniken wurden Patienten für die Befragung rekrutiert:

- Universitätsklinikum Göttingen (Abteilung für Hämatologie und Onkologie und Abteilung für Strahlentherapie),

- Evangelisches Krankenhaus Weende (Abteilung für Chirurgie),

- Krankenhaus Lenglern (Abteilung für Pneumonologie).

Weitere Patienten wurden mit Hilfe der onkologischen Praxis Meyer/Ammon in Göttingen rekrutiert. Bedingung war, dass auch diese Patienten im jeweils vorangegangenen Jahr in den oben genannten Kliniken behandelt wurden.

Die Göttinger Abteilung für Ethik und Geschichte der Medizin versendete Fragebögen an die jeweils in Frage kommenden Patienten. Vor dem Versand wurde, wie von der Ethikkommission gefordert, durch telefonischen Kontakt mit den behandelnden Hausärzten der Gesundheitszustand der Patienten evaluiert bzw. erfragt, ob die Patienten gesundheitlich dazu in der Lage seien, einen solchen Fragebogen auszufüllen. Traf dies zu, wurden die Patienten in die Liste der teilnehmenden Patienten eingetragen.

Die Adresse des jeweiligen Patienten wurde mit einer Nummer versehen und so für die spätere Auswertung pseudonymisiert. Die Nummer wurde auf dem beiliegendem Rückumschlag vermerkt, so dass es später möglich war, die teilnehmenden Patienten bei eventuell auftretenden Rückfragen zu kontaktieren.

Der versendete Umschlag hatte folgenden Inhalt:

- Anschreiben des jeweils behandelnden Klinikarztes mit Erläuterungen zu der Studie,

- Fragebogen,

- Einverständniserklärung,

- frankierter Rückumschlag. 


\subsubsection{Wahl des Zeitpunktes der empirischen Datenerhebung und Einschlusskriterien der Befragten}

Der Befragungszeitraum wurde so gewählt, dass die Angaben der Patienten sowohl den Zeitpunkt vor als auch den Zeitraum während der Einführung des DRG-Abrechnungssystems abbilden konnten. Dadurch wurde ein Vergleich der Daten vor und während der Einführung der fallpauschalisierten Abrechnung möglich. Im ersten Jahr der Studie (2004) befragten wir Patienten, die im Jahr 2003 im Rahmen des alten Abrechnungssystems stationär behandelt wurden. Die Befragung in den beiden darauffolgenden Jahren bezog sich auf Patienten, die während der Einführung des neuen Systems in stationärer Behandlung waren.

Die Abteilung für Ethik und Geschichte der Medizin der Universität Göttingen führte die Datenerhebung jeweils in den Monaten Februar bis Mai 2004-2006 durch.

Das befragte Kollektiv bestand aus Patienten mit Bronchialkarzinom. Der Begriff „Bronchialkarzinom“ vereinigt unterschiedliche Arten von Lungentumoren. Sie lassen sich zunächst in kleinzellige Lungentumoren (SCLC - small cell lung cancer) und nichtkleinzellige Lungentumoren (NSCLC - non small cell lung cancer) unterteilen. Zu den NSCLC gehören das Adeno- und Plattenepithelkarzinom sowie das großzellige Karzinom. Das kleinzellige Karzinom wird auch Haferzellkarzinom genannt und ist ein extrem schnell und invasiv wachsender Tumor. Da die Gruppe der antwortenden Patienten nicht sehr groß war und die Patienten trotz unterschiedlicher Tumorarten im jeweils gleichen institutionellen Setting behandelt wurden, verzichteten wir auf eine Unterteilung nach Art des Bronchialkarzinoms.

Wie bereits in der Einführung erwähnt ist aus ethischer Sicht insbesondere die Untersuchung der Veränderungen in der Behandlung von onkologischen Patienten interessant. Diese Patienten benötigen sehr teure Medikamente, deren Abbildung in einem pauschalisierten System schwierig erscheint. Außerdem sind es oft schwerkranke Patienten, die eine besondere menschliche Fürsorge benötigen. Bei Bronchialkarzinompatienten kommt hinzu, dass ein großer Teil dieser Patienten Raucher ist (s. Tabelle 3.1.2a), und Schuldvorwürfe auf sich zieht. Knappe Ressourcen könnten bei dieser Patientengruppe zu einer Minderversorgung führen, wenn die Bereitschaft der Gesellschaft abnimmt, die Kosten für selbst verschuldete Krankheiten zu tragen. Aus diesen Gründen ist die Untersuchung von Patienten mit Bronchialkarzinom besonders für die 
Datenerhebung dieser Studie geeignet. Sie dient dazu, die Einführung von Fallpauschalen exemplarisch an einer betroffenen Patientengruppe zu untersuchen.

Als Problem der Befragung dieses Kollektivs stellte sich deren hohe Mortalitätsrate (5-JahresÜberlebensrate um 15\% (Macha 2003)) heraus. Aufgrund der hohen Sterblichkeitsrate konnte schon zu Beginn eines jeweiligen Jahres nur noch rund die Hälfte der zunächst ermittelten Patienten an der Studie teilnehmen. Dabei wurden (nach Einschätzung des Hausarztes) schwerkranke Patienten nicht durch das Studienteam kontaktiert, um unzumutbare studienbedingte Belastungen zu vermeiden.

\title{
2.2.2. Patientenfragebogen
}

Der Fragebogen wurde vom Institut für Sozialmedizin, Epidemiologie und Gesundheitsökonomie in Berlin in Zusammenarbeit mit der Abteilung für Ethik und Geschichte der Medizin der Universität Göttingen entworfen. Um die Fragen möglichst praxisnah zu halten, war neben diesen beiden Instituten auch die Abteilung Hämatologie und Onkologie des Universitätsklinikums Göttingen an der Fragebogenkonzeption beteiligt. Die Datenerhebung erfolgte durch eine schriftliche Befragung von Lungenkrebspatienten. Der Fragebogen enthielt sowohl offenen Fragen als auch Fragen mit vorgegebenen quantitativen und qualitativen Antwortmöglichkeiten. Der Fragebogen gliedert sich in sechs Abschnitte:

\author{
I. Allgemeine Fragen \\ II. $\quad$ Ärztliche Betreuung \\ III. Ärztliche Versorgung \\ IV. Erwerbstätigkeit \\ V. Pflege \\ VI. Standardisierter Fragebogen V EORTC QLQ-C30 (version 3)
}

Die Daten der vorliegenden Studie generieren sich aus den Abschnitten (I), (II) und (VI). Diese drei Abschnitte haben für die ethische Untersuchung unter den Aspekten der Gerechtigkeit und des Nichtschädigungsprinzips die größte Relevanz. Sie werden im Folgenden näher vorgestellt. 


\section{Zu (I)}

Um einen Überblick über das befragte Kollektiv zu bekommen, erfasst der erste Abschnitt soziodemographische Daten der Patienten. Dazu gehören Geburtsjahr, Geschlecht, Größe, Gewicht, höchster Bildungsabschluss und Rauchgewohnheiten. Die Fragen zielen darauf ab, die demographischen und persönlichen Eigenschaften der Studienteilnehmer zu erfassen. Zum Vergleich des Kollektivs ist in der Literatur eine Studie beschrieben, in der über 13 Jahre die Population der Bronchialkarzinompatienten untersucht wurde: „Lung cancer in North-West Italy: demographic and clinical trends in a hospital-based population of 1277 patients“ (Buccheri, Ferrigno 2003) Diese Studie zeigt Übereinstimmungen der soziodemographischen Daten der italienischen Patienten mit der eigenen untersuchten Patientenpopulation. Allerdings soll schon hier darauf hingewiesen werden, dass die Ergebnisse dieser Studie keinen Anspruch auf Allgemeingültigkeit erheben.

\section{Zu (II)}

Der zweite Teil des Fragebogens bezieht sich auf die Beurteilung der ärztlichen und pflegerischen Betreuung. Wie bereits in der Einleitung erläutert, soll in der vorliegenden Studie geprüft werden, ob es während der DRG-Einführung Veränderungen in der stationären Behandlung gibt, die Auswirkungen auf das Nichtschädigungsprinzip und das Prinzip der Gerechtigkeit haben. Der zweite Abschnitt des Fragebogens untersucht diesen Aspekt aus Sicht der Patienten, indem Fragen zur ärztlichen und pflegerischen Behandlung und deren Qualität gestellt werden.

In Frage (1) wird der Zeitpunkt der Diagnosestellung erfasst. Die Angabe erfolgt in Monat und Jahr.

Die folgenden sieben Fragen sollen Aufschluss über die individuellen Beurteilungen des Gesprächs zwischen behandelndem Arzt und Patient geben. Dabei werden sowohl geschlossene, als auch offene Fragen gestellt.

Frage (2) Das Gespräch, in dem mir die Diagnose Lungentumor mitgeteilt wurde, dauerte _ Minuten 


\begin{tabular}{|l|l|}
\hline $\begin{array}{l}\text { Frage (3) Hat der behandelnde Arzt mit Ihnen verschiedene Möglichkeiten der } \\
\text { Behandlung Ihrer Krankheit durchgesprochen? }\end{array}$ \\
\hline & Nein \\
\hline & Ja, nämlich: \\
\hline & Chemotherapie \\
\hline & Bestrahlung \\
\hline & Operative Entfernung \\
\hline & Kombination aus diesen \\
\hline
\end{tabular}

Frage (4) Hatten Sie den Eindruck, dass der behandelnde Arzt auf Ihre Wünsche und Bedürfnisse eingegangen ist?

\begin{tabular}{|l|l|l|l|l|}
\hline Überhaupt nicht & Etwas & Mäßig & Ziemlich & Sehr \\
\hline & & & & \\
\hline
\end{tabular}

\begin{tabular}{|l|l|}
\hline Frage (5) Wurden Sie über die Wirkungsweise Ihrer Behandlung aufgeklärt? \\
\hline & Nein \\
\hline & Ja \\
\hline
\end{tabular}

Frage (6) Wurden Sie über mögliche Nebenwirkungen Ihrer Behandlung aufgeklärt?

\begin{tabular}{|l|l|}
\hline & Nein \\
\hline & Ja \\
\hline
\end{tabular}


Frage (7) Der Arzt hat mit Ihnen vor der Behandlung ein Gespräch durchgeführt und Ihnen alles erklärt. Wie viel von seinen Erklärungen haben sie verstanden?

\begin{tabular}{|l|l|l|l|l|}
\hline Gar nichts & Wenig & Die Hälfte & Vieles & Alles \\
\hline & & & & \\
\hline
\end{tabular}

\section{Frage (8) Was könnte für die Zukunft an solchen Gesprächen verbessert werden?}

Bei Frage (8) haben die Studienteilnehmer wie bei Frage (4) die Möglichkeit, Freitextanmerkungen zu machen.

Mit Hilfe der Fragen (9)-(11) beurteilen die Befragten die ärztliche und pflegerische Betreuung und die Zusammenarbeit von Haus- und Klinikärzten. Die Beurteilung findet jeweils auf einer Likert-Skala mit fünf Abstufungen statt (5 = ,ungenügend“ ; $1=$,,sehr gut“).

Diese Beurteilung ist in Hinblick auf die Veränderungen, die sich durch die Einführung von Fallpauschalen ergeben können, besonders interessant, da sie einen Rückschluss auf die Qualität der Behandlung vor und während der Einführung zulässt.

Frage (12) und (13) geben Auskunft über die Sorgen und Ängste der Befragten.

\begin{tabular}{|l|l|}
\hline \multicolumn{2}{|l|}{$\begin{array}{l}\text { Frage (12) Mit folgenden Personen konnte ich mich während der Behandlung über meine } \\
\text { Ängste und Sorgen unterhalten: }\end{array}$} \\
\hline & Mit niemandem. \\
\hline & Mit Bekannten und Freunden. \\
\hline & Mit dem Hausarzt. \\
\hline & Mit dem niedergelassenen Facharzt. \\
\hline & Mit den behandelnden Ärzten im Krankenhaus / im Klinikum. \\
\hline
\end{tabular}




\begin{tabular}{|l|l|}
\hline Frage (13) Welche Sorgen hatten sie bei der Entlassung aus dem Krankenhaus? \\
\hline & Keine. \\
\hline & Wegen der Versorgung zu Hause. \\
\hline & Wegen der zusätzlichen Belastung meiner Angehörigen. \\
\hline & Wegen Einsamkeit und Alleinsein. \\
\hline
\end{tabular}

Auch zu Frage (13) konnten die Studienteilnehmer eigene Bemerkungen aufschreiben.

Frage (14) befasst sich mit der Versorgung und Pflege nach Entlassung aus dem Krankenhaus. Diese Frage ist im Hinblick auf die Studien aus den USA besonders interessant. Wie in der Einführung erwähnt, nimmt der Pflegebedarf nach Entlassung signifikant zu (s. 1.4.3). In Frage (14) macht das eigene Patientenkollektiv Angaben über die weitere Art Versorgung bzw. Pflege nach Entlassung. Dadurch können Aussagen über eine mögliche Veränderung des Pflegebedarfs während der Einführung des DRG-Systems getroffen werden.

Die letzten zwei Fragen dieses Abschnittes Frage (15) und (16) werden unter dem Gesichtspunkt der gerechten Verteilung gestellt. Es wird nach der Länge des Anfahrtsweges (in Kilometern) gefragt (15) und welche Streckenlänge der Patient bei seinem derzeitigen Gesundheitszustand für angemessen hält (16). Wie in der Einleitung beschrieben wird der Trend von Klinikzusammenlegungen zu spezialisierteren Krankenhäusern prognostiziert, so dass sich der durchschnittliche Anfahrtsweg verlängert, wenn nicht mehr in jeder (kleineren) Klinik onkologische Krankheitsbilder behandelt werden. Dieser Trend kann dazu führen, dass das Einzugsgebiet der an der Studie teilnehmenden Kliniken größer wird. Die Gerechtigkeitsfrage ist dann wichtig, wenn Patienten aus einer ländlichen Region weite Anfahrtswege haben, die ihnen mit ihrem Gesundheitszustand nicht mehr zumutbar sind. Es würde so zu einer Benachteiligung der onkologischen Patienten in ländlichen Gebieten kommen.

Die Auswertung der Daten aus den Abschnitten (I) und (II) wurde mit Hilfe von Excel-Tabellen durchgeführt. Die Daten wurden mit einem Zahlencode verschlüsselt und in Tabellen 
eingetragen. Danach wurden jeweils Median und Mittelwert gebildet. Die Auswertung erfolgte vergleichend für alle drei Jahre.

Nach Rücksprache mit dem Institut für Medizinische Statistik der Universität Göttingen wurde von einer weiteren statistischen Auswertung der Daten abgesehen, da durch die Art der Daten und die geringe Rücklaufquote keine Signifikanz der antwortenden Population auf die Grundgesamtheit der Bronchialkarzinompatienten erzielt werden kann. Die gewonnenen Daten erlauben daher keine allgemeingültigen Aussagen. Die Auswertung der Daten wird beschreibend und nicht mit Hilfe eines Signifikanztestes durchgeführt. Auf die Problematik, die sich dadurch für die Aussage ergibt, wird in der Diskussion eingegangen.

Die erhobenen Daten wurden zur deskriptiven Auswertung in Tabellen eingetragen. Sie wurden vergleichend für die Zeit vor und während der Einführung des DRG-Systems ausgewertet.

Die Freitextanmerkungen der Patienten wurden mit Hilfe induktiver Kategorienbildung ausgewertet. Dabei handelt es sich um eine qualitative Inhaltsanalyse, die „eine Ableitung von Auswertungsgesichtspunkten aus dem Material heraus“ ermöglicht (Mayring 2002, S. 86).

\section{Zu (VI)}

Im Teil (VI) des Fragebogens wurde die Lebensqualität der Patienten zum Zeitpunkt der Befragung mit Hilfe des Standardfragebogens EORTC QLQ-C30 (version 3) untersucht. Dieser Fragebogen ist von der „European Organization for Research and Treatment of Cancer“ (EORTC) zur Erfassung der Lebensqualität von Patienten mit onkologischen Krankheitsbildern entwickelt worden. ${ }^{2}$ Die Erfassung der Lebensqualität ist für die vorliegende Studie von besonderer Bedeutung, da sie ein Messkriterium für die Veränderungen während der Einführung von DRG ist (s. Kap. 1.5.3.). Es erscheint sinnvoll, den Patienten eine Selbsteinschätzung ihrer Lebensqualität zu ermöglichen, da sie als unmittelbar Betroffene am besten ihre Verfassung beurteilen können.

Der Fragebogen QLQ-C30 ist ein Standardfragebogen in Europa, um die Lebensqualität onkologischer Patienten darzustellen. Er umfasst insgesamt 30 Fragen (Items), die sich in sechs Funktions- und neun Symptomskalen unterteilen lassen.

\footnotetext{
${ }^{2}$ Die Nutzung dieses Fragebogens erfolgte mit freundlicher Genehmigung der EORTC in Brüssel.
} 


\section{Funktionsskalen}

körperlicher Funktionszustand (Fragen 1-5)

Rollenfunktion (Fragen 6,7)

Seelischer Zustand (Fragen 21-24)

Kognitiver Zustand (Fragen 20, 25)

Soziale Kontakte (Fragen 26, 27)

Globale körperliche Aktivität/Lebensqualität (Fragen 29, 30)

\section{Symptomskalen}

Müdigkeit (Fragen 10, 12, 18)

Übelkeit und Erbrechen (Fragen 14, 15)

Schmerzen (Fragen 9, 19)

Dyspnoe (Frage 8)

Schlaflosigkeit (Frage 11)

Appetitverlust (Frage 13)

Obstipation (Frage 16)

Diarrhoe (Frage 17)

Finanzielle Schwierigkeiten (Frage 28)

Als Antwortschema werden die Antwortmöglichkeiten in vier Abstufungsmöglichkeiten angegeben. ( 1 = überhaupt nicht; 2 = wenig; 3 = mäßig; 4 = sehr). Für die Beantwortung der Fragen zur globalen körperlichen Aktivität bzw. Lebensqualität (Fragen 29 und 30) gibt es sieben Abstufungsmöglichkeiten (von 1 = sehr schlecht bis 7 = ausgezeichnet).

Die Skala „finanzielle Beeinträchtigung“ wird als Symptom gewertet, da der Fragebogen QLQC30 international angewendet wird und es in anderen Ländern in Folge einer onkologischen Erkrankung durchaus zu dem Symptom ,finanzielle Beeinträchtigung“ kommen kann.

Um die Fragebögen deskriptiv auszuwerten, werden die Rohdaten entsprechend der Vorgaben der EORTC transformiert. Dazu werden die angegebenen Werte innerhalb einer Skala summiert und danach durch die Anzahl der Items der jeweiligen Skala geteilt. So erhält man den Durchschnittswert einer Skala. Sechs der Symptomskalen bestehen nur aus einem Item. Aus Gründen der Übersichtlichkeit soll aber der Begriff „Skala“ erhalten bleiben. 
Der errechnete Durchschnittswert $(\mathrm{RS}=$ raw score) der jeweiligen Skala wird mittels folgender Formeln linear transformiert:

Skala der ,allgemeinen Funktion“

$$
\mathrm{S}=(1-(\mathrm{RS}-1) / \text { range }) * 100
$$

Skala der ,allgemeinen Symptome“

Skala des „,allgemeinen Gesundheitsstatus / Lebensqualität“

$$
\mathrm{S}=((\mathrm{RS}-1) / \text { range })^{*} 100
$$

$\mathrm{S}=$ Skalenpunktwert

Range $=$ Bereich zwischen größtem möglichen und kleinstem möglichen Wert

Das Ergebnis dieser Formel, der Skalenpunktwert, bewegt sich für jede Skala zwischen 0-100. Ein hoher Punktwert in den Funktionsskalen bedeutet ein hohes Maß an „Funktionieren“ und somit eine gute Lebensqualität. Ein hoher Punktwert in den Symptomskalen bedeutet ein hohes $\mathrm{Maß}$ an Symptomen, also starke Beschwerden und eine geringere Lebensqualität. Fehlende Werte werden bei dieser Umrechnung in einem gewissen Rahmen akzeptiert. Wenn 50\% der Fragen eines Items beantwortet sind, kann die Subskala ausgewertet werden.

Die Aussagekraft dieses standardisierten Fragebogens ist durch die methodischen Gütekriterien Validität (Gültigkeit), Reliabilität (Zuverlässigkeit) und Sensitivität in der Literatur bestätigt worden (Aaronson et al. 1996).

Die Rohdaten und transformierten Daten wurden tabellarisch gespeichert und deskriptiv ausgewertet.

\subsection{Durchführung der Interviews mit Ärzten}

Neben der schriftlichen Patientenbefragung wurden im Rahmen der vorliegenden Studie von der Autorin persönliche, teilstrukturierte Interviews mit Ärzten und Pflegekräften durchgeführt. Wir haben diese Befragungsart gewählt, um besonders auf die individuellen Erfahrungen der Befragten eingehen zu können. Die Anzahl der befragten Ärzte und Pflegekräfte war im 
Gegensatz zu der Patientenbefragung so gewählt, dass im Rahmen dieser Studie die Durchführung persönlicher Interviews möglich war. Vorteile dieser Art der Datenerhebung sind:

- Kontrollierbare Datenerhebung;

- spontane Antwortmöglichkeiten;

- Sicherheit darüber, wer die Antwort gibt;

- der Befragte kann innerhalb seines eigenen Referenzsystems antworten, ohne in eine bestimmte Richtung gelenkt zu werden;

- offene Fragen unterstützen Äußerungen, die auch „tatsächlich“ im Einstellungsrahmen des Befragten verankert sind (Schnell et al. 1999).

Um zu gewährleisten, dass die an der Befragung teilnehmenden Ärzte eine Veränderung seit Einführung der DRG beurteilen konnten, sollten sie mindestens seit Anfang 2003 mit der Versorgung von Patienten mit Bronchialkarzinom vertraut sein. Die Befragten waren sowohl als Chef-, wie auch als Ober- und Assistenzärzte tätig und in unterschiedlichen Abteilungen beschäftigt. Sie arbeiteten in einer der drei an der Studie teilnehmenden Kliniken. Alle befragten Ärzte waren männlich. Insgesamt wurden zwölf Ärzte in persönlichen Interviews befragt. Die Befragung fand im Zeitraum von November 2005 bis Januar 2006 statt. Im Vorfeld der persönlichen Interviews wurde ein Termin mit den jeweiligen Chefärzten der Abteilung bzw. deren Stellvertretern vereinbart, um die Rahmenbedingungen und das Setting der Befragung zu klären. Außerdem wurde den befragten Ärzten das Thema der Befragung mitgeteilt, so dass diese sich bereits vor den eigentlichen Interviews auf das Thema einstellen konnten.

Die Interviews dauerten zwischen 10 und 20 Minuten und wurden auditiv aufgezeichnet. Die Befragung fand in einem dafür zugewiesenen Raum in ruhiger Umgebung statt. Es erfolgte eine schriftliche Bearbeitung der Aufnahmen nach Durchführung der Interviews. Dabei wurden die auditiven Daten stichwortartig niedergeschrieben und einer qualitativen Inhaltsanalyse unterzogen. Zahlenwerte wurden für jedes der teilnehmenden Häuser gesondert errechnet. Ähnliche Antworten bzw. gleiche Meinungen wurden in Gruppen gegliedert, so dass eine systematische Typisierung der Angaben und Meinungen zur Auswertung geführt hat. 
Aus dieser stichprobenartigen Befragung wurden subjektive Beurteilungen der Ärzte gewonnen. Sie fließen als Ergänzung zu den Patientendaten und den übrigen Daten zur Beantwortung der forschungsleitenden Fragen in die vorliegende Studie ein. Nachfolgend wird der Fragebogen vorgestellt, der als Leitlinie für die teilstrukturierten Interviews vorlag. Einige Fragen sind dabei als offene Fragen konzipiert.

\subsubsection{Fragenkatalog}

Der Fragebogen wurde von der Abteilung für Ethik und Geschichte der Medizin in Zusammenarbeit mit der Abteilung für Hämatologie und Onkologie des Universitätsklinikums Göttingen entwickelt. Dabei wurde der Fragebogen durch die onkologische Abteilung auf fachlich wichtige und korrekte sowie logische Fragestellung überprüft. Anhand des Fragebogens sollen das DRG-System und seine Einführung aus ärztlicher Sicht bewertet werden.

Im ersten Teil des Fragebogens werden die soziodemographischen Parameter der Befragten erfasst. Neben Geburtsjahr, Geschlecht und Berufsbezeichnung wird auch gefragt, wie lange die Person in der jeweiligen Abteilung tätig ist. Aus diesen Daten wird die Dauer der Erfahrung der befragten Person im Umgang mit Bronchialkarzinompatienten ersichtlich.

Die folgenden Fragen geben Auskunft über Arbeitsbedingungen vor und nach der DRGEinführung, außerdem geben die Studienteilnehmer allgemeine Informationen zur Arbeit mit Bronchialkarzinompatienten. Die letzte Frage des Fragenkatalogs bezieht sich auf die persönliche Meinung des Befragten zur DRG-Einführung.

\subsubsection{Fragenkatalog der teilstrukturierten Interviews}

1. Sind Sie der Meinung, dass sich seit dem 1. Januar 2004 (flächendeckende Einführung der Abrechnung nach DRG) etwas an Ihren Arbeitsbedingungen verändert hat? Und wenn ja, wie sehen diese Veränderungen aus?

2. Inwieweit hat sich diese eventuelle Veränderung im letzten Jahr noch verschärft?

3. Haben sich durch die Einführung der DRG Entlassungs- oder Aufnahmekriterien für Patienten geändert? Wenn ja, wie sieht eine solche Veränderung aus? 
4. Gibt es seit der Einführung deutlich spürbarere Unterschiede in der Behandlung von Kassenpatienten und Selbstzahlern?

5. Wie viel Zeit nehmen Sie sich durchschnittlich für das erste Gespräch, in dem Sie einem Patienten eine Krebsdiagnose mitteilen?

6. Führen Sie nach dem ersten Aufklärungsgespräch noch weitere Gespräche über die Konsequenzen der Diagnose mit dem Patienten?

7. Werden auf Ihrer Station die Patienten mit Bronchialkarzinom nach speziellen Behandlungsrichtlinien versorgt? Wenn ja, wie sehen diese Richtlinien aus?

8. Ergänzung: Gibt es feste Behandlungsrichtlinien bei anderen onkologischen Diagnosen?

9. Über welche Behandlungsalternativen sprechen Sie mit einem Lungenkrebspatienten?

10. Konnten Sie vor Einführung der DRG auf individuelle Wünsche des Patienten eingehen? Wenn ja, wie sahen diese Wünsche aus?

11. Gibt es zu Frage 10 Veränderungen seit der DRG-Einführung?

12. Gibt es seit der DRG-Einführung 2004 andere Behandlungsoptionen bzw. neue diagnostische Verfahren für Patienten mit Lungenkrebs? Und wie sehen diese aus?

13. Gibt es seitdem Veränderungen in den Behandlungsergebnissen von Lungenkrebspatienten und somit eine Veränderung der Prognose?

14. Wie schätzen Sie derzeit die Zusammenarbeit mit den Hausärzten in Bezug auf die Weiterbehandlung der Patienten ein?

15. Wie beurteilen Sie als Arzt die Einführung des DRG-Systems?

Diese Fragen dienen unter Berücksichtigung aller übrigen erhobenen Daten dazu, ein umfassendes Bild über die Veränderungen der stationären Behandlung von Bronchialkarzinompatienten im Raum Südniedersachsen während der Einführung des DRGSystems zu gewinnen. Es findet ein Vergleich mit den Daten aus der Patientenbefragung statt, um die in den persönlichen Interviews gewonnenen Angaben entweder zu bestätigen oder unterschiedliche Wahrnehmungen festzustellen. Die Antworten geben über die subjektive Einschätzung der Ärzte Auskunft. 


\subsection{Durchführung der Interviews mit Pflegekräften}

Analog zu den Interviews mit Ärzten wurden auch persönliche, teilstrukturierte Interviews mit Pflegekräften von der Autorin durchgeführt. Dazu wurden 13 Pflegekräfte (9 weiblich, 4 männlich) befragt. Die Befragung fand in den an der Studie teilnehmenden Kliniken im April 2006 statt.

Einschlusskriterien für die teilnehmenden Pflegekräfte waren die Beschäftigung in einer der drei teilnehmenden Kliniken, Erfahrung im Umgang mit Bronchialkarzinompatienten und das Ausführen der pflegerischen Tätigkeit seit mindestens Januar 2003. Auch bei dieser Befragung wurde zunächst mit den Pflegeleitungen ein Termin vereinbart und darauf Pflegekräfte für die Interviews rekrutiert. Die Interviews fanden in einer dafür angemessen ruhigen Umgebung statt. Der Fragenkatalog wurde zum größten Teil aus der Befragung der Ärzte übernommen. Insgesamt wurden 17 Fragen gestellt. Neu hinzugekommen bzw. modifiziert wurden folgende Fragen:

\subsubsection{Modifizierter Fragenkatalog}

- Sind Sie mit Ihren jetzigen Arbeitsbedingungen zufrieden? Hat sich daran in den letzten Jahren etwas geändert? Wenn ja, was hat sich verändert?

- Sind Fallpauschalen ein Gesprächsthema, über das Sie mit Ihren Kollegen aus der Pflege, mit Ärzten oder Vertretern der Verwaltung reden?

- Haben sich Zeitabläufe auf der Station geändert? Wenn ja, wie haben sie sich verändert?

- Wie viel Zeit können Sie sich für Patienten nehmen und gibt es Raum für Gespräche?

- Bekommen Sie ein Feedback von den Patienten? Wenn ja, wie sieht dieses aus?

- Gibt es von Seiten der Pflege Neuerungen für die Pflege von Bronchialkarzinompatienten seit 2004? Wenn ja, welche?

- Wie schätzen Sie die Zusammenarbeit von Ärzten und Pflegekräften in Bezug auf die Patientenversorgung ein?

- Wie beurteilen Sie als Pflegekraft die Einführung des DRG-Systems? 
Die Interviews wurden von der Autorin persönlich durchgeführt und auditiv aufgezeichnet. Danach erfolgte analog zu der Bearbeitung der Interviews mit Ärzten eine schriftliche Aufarbeitung der erhobenen Daten durch eine systematische Typisierung der Antworten. Zur Beantwortung der forschungsleitenden Fragen wurden die Aussagen der Pflegekräfte mit denen der Patientenbefragung und der Interviews mit Ärzten in Zusammenhang gesetzt.

\subsection{Erfassung der Controlling-Daten des Universitätsklinikums Göttingen}

Neben den Ergebnissen aus den schriftlichen Befragungen und den persönlichen Interviews sind auch Daten der Stabsstelle Controlling der Universitätsklinik Göttingen für die vorliegende Studie ausgewertet worden. Es wurden Daten aus den Jahren 2003-2005 ermittelt, also aus dem Zeitraum, über den auch die übrigen erhobenen Daten Aussagen machen. Ausgewertet wurden die Daten aller Patienten, die in diesem Zeitraum im Universitätsklinikum Göttingen mit der Diagnose „Bronchialkarzinom“ stationär behandelt wurden. Wichtig für die Auswertung und das Verständnis der Daten ist eine Unterscheidung zwischen „Fällen“ und „Patienten“. Ein Patient kann mehr als einmal in einem Jahr mit der gleichen Diagnose aufgenommen werden und wird dann als neuer „Fall“ gesehen. Deshalb beziehen sich die folgenden Daten auf die Anzahl der Fälle und nicht auf die Anzahl der Patienten. Folgende Aspekte sind für die Beantwortung der Forschungsfragen wichtig:

- Fallzahl: Anzahl der Fälle, die mit der Diagnose „Bronchialkarzinom“ behandelt wurden;

- Mittlere Verweildauer: durchschnittliche Zeit (in Tagen), die ein Patient als einzelner Fall stationär behandelt wurde;

- Anzahl der Kurzlieger: Anzahl der Fälle, deren Aufenthaltsdauer unter der Grenzverweildauer ${ }^{3}$ liegt;

- Anzahl der Langlieger: Anzahl der Fälle, deren Aufenthaltsdauer über der Grenzverweildauer liegt;

- Letalitätsrate: Sterblichkeitsrate im Krankenhaus;

\footnotetext{
${ }^{3}$ Grenzverweildauer: im DRG-System für jede Diagnose festgelegte Dauer, die eine stationäre Behandlung weder über- noch unterschreiten soll
} 


\section{MATERIAL UND METHODEN}

- Relativgewicht: Durchschnittswert der Rohfallkosten ${ }^{4}$ einer Fallgruppe dividiert durch den Durchschnittswert der gesamten Stichprobe;

- Effektivgewicht: wird mit dem Basisfallpreis ${ }^{5}$ multipliziert, um den Fallerlös zu errechnen (berücksichtigt Zu-bzw. Abschläge);

- Effektiverlös: Fallerlös addiert zu evtl. anfallenden Zusatzentgelten;

- Erlösanteil aus Zusatzentgelten: Zusätzliche Gelder, die außerhalb des DRG-Systems errechnet werden.

\footnotetext{
${ }^{4}$ Rohfallkosten: vollständige Verteilung der DRG-relevanten Kosten unter der Berücksichtigung der DRGrelevanten Leistungen auf die DRG-Fälle

${ }^{5}$ Basisfallpreis: von der InEK festgelegter Preis für die durchschnittlichen Kosten einer Krankenhausbehandlung (zu Beginn klinikintern, am Ende der Konvergenzphase (bundes)landweit festgelegt)
} 


\section{DARSTELLUNG DER UNTERSUCHUNGS- ERGEBNISSE}

\subsection{Auswertung der Patientenfragebögen}

Um die vermuteten Veränderungen in der Behandlung und der Lebensqualität des untersuchten Patientenkollektivs sichtbar zu machen, werden die Daten aus den Patientenbefragungen 20042006 miteinander verglichen. Die Daten, die 2004 erhoben wurden, beziehen sich auf das Jahr 2003 und somit auf die stationären Bedingungen vor Einführung von Fallpauschalen. Die Daten aus den Jahren 2005 und 2006 beziehen sich jeweils auf das vorangegangene Jahr. Sie spiegeln die Bedingungen während der DRG-Einführung wider.

\subsubsection{Stichprobengröße der Patientenbefragung}

Insgesamt wurden in den Jahren 2004-2006 403 Patienten angeschrieben. Von diesen 403 angeschrieben Patienten haben 109 verwendbare Fragebögen zurückgesendet. Die Rücklaufquote beträgt damit 27\%. Für die einzelnen Jahre ergibt sich bei einer Personenzahl von $\mathrm{N}=109$ jeweils folgender Stichprobenumfang:

\begin{tabular}{|l|l|l|l|}
\hline Jahr des Klinikaufenthalts & $\mathbf{2 0 0 3}$ & $\mathbf{2 0 0 4}$ & $\mathbf{2 0 0 5}$ \\
\hline Anzahl der auswertbaren Fragebögen & 41 & 37 & 31 \\
\hline
\end{tabular}

Tabelle 3.1.1 Stichprobenumfang der Patientenbefragung 2003-2005

\subsubsection{Beschreibung des Patientenkollektivs}

Um einen Überblick über das befragte Kollektiv zu bekommen, werden im ersten Abschnitt soziodemographische Daten erhoben. Dazu gehören Geburtsjahr, Geschlecht, Größe, Gewicht, Ausbildungsabschluss und Rauchgewohnheiten. Es haben 76 Männer und 32 Frauen geantwortet. Ein Patient macht keine Angaben zu seinem Geschlecht. Der Altersmedian beträgt 66 Jahre, 24 


\section{DARSTELLUNG DER UNTERSUCHUNGSERGEBNISSE}

Patienten von 109 (22\%) sind Nichtraucher. Tabelle 3.1.2a vergleicht die soziodemographischen Daten der Jahre 2003 bis 2005 miteinander.

\section{Angaben zur eigenen Person}

\begin{tabular}{|l|l|l|l|l|}
\hline Jahr & $\mathbf{2 0 0 3}$ & $\mathbf{2 0 0 4}$ & $\mathbf{2 0 0 5}$ & $\mathbf{2 0 0 3 - 2 0 0 5}$ \\
\hline $\begin{array}{l}\text { Frauen } \\
\text { (Personenzahl) }\end{array}$ & 10 & 13 & 9 & 32 \\
\hline $\begin{array}{l}\text { Männer } \\
\text { (Personenzahl) }\end{array}$ & 31 & 24 & 21 & 76 \\
\hline $\begin{array}{l}\text { Altersmedian } \\
\text { (in Jahren) }\end{array}$ & 67 & 65 & 67 & 66 \\
\hline $\begin{array}{l}\text { Nichtraucher } \\
\text { (Personenzahl) }\end{array}$ & 13 & 5 & 6 & 24 \\
\hline
\end{tabular}

Tabelle 3.1.2a Vergleich der soziodemographischen Patientendaten 2003-2005

Tabelle 3.1.2b gibt einen Überblick über den höchsten Bildungsabschluss der befragten

Patienten. $73 \%$ der Studienteilnehmer haben einen Hauptschulabschluss und 20\% haben die Schule mit mittlerer Reife verlassen. Nur 3\% haben Abitur gemacht und 5\% einen Hochschulabschluss.

\section{Schulbildung (höchster Abschluss)}

\begin{tabular}{|l|l|l|l|l|}
\hline Jahr & $\mathbf{2 0 0 3}$ & $\mathbf{2 0 0 4}$ & $\mathbf{2 0 0 5}$ & $\mathbf{2 0 0 3 - 2 0 0 5}$ \\
\hline $\begin{array}{l}\text { Hauptschule } \\
\text { (Personenanzahl) }\end{array}$ & 36 & 20 & 21 & $77(73 \%)$ \\
\hline $\begin{array}{l}\text { Realschule } \\
\text { (Personenanzahl) }\end{array}$ & 4 & 10 & 7 & $21(20 \%)$ \\
\hline $\begin{array}{l}\text { Abitur } \\
\text { (Personenanzahl) }\end{array}$ & 0 & 3 & 0 & $3(3 \%)$ \\
\hline $\begin{array}{l}\text { Hochschule } \\
\text { (Personenanzahl) }\end{array}$ & 1 & 2 & 2 & $5(5 \%)$ \\
\hline $\begin{array}{l}\text { Gesamtzahl } \\
\text { (Personenanzahl) }\end{array}$ & 41 & 35 & 30 & $106(100 \%)$ \\
\hline
\end{tabular}

Tabelle 3.1.2b Vergleich höchster Bildungsabschluss der Patienten ${ }^{7}$

\footnotetext{
${ }^{6} \mathrm{Zu}$ der Tabelle ist anzumerken, dass im Jahr 2005 ein Befragter keine Angaben zur eigenen Person gemacht hat. Im Jahr 2004 haben zwei der Befragten keine Angaben zur Schulbildung gemacht.

${ }^{7}$ Durch Runden der Prozentzahlen kommt es in der Summe zu einem Wert $>100 \%$.
} 


\subsubsection{Repräsentativität der Daten}

Um die Vergleichbarkeit der Patienten dieser Studie mit einer größeren Population von Bronchialkarzinompatienten zu zeigen, wurde eine Studie herangezogen, in der über 13 Jahre die Population der Bronchialkarzinompatienten untersucht wurde: „Lung cancer in North-West Italy: demographic and clinical trends in a hospital-based population of 1277 patients“ (Buccheri, Ferrigno 2003). In diese Studie wurden alle in einer italienischen Klinik auf Grund ihres Bronchialkarzinoms behandelten Patienten eingeschlossen $(\mathrm{N}=1277)$. Es ergeben sich folgende Gemeinsamkeiten bzw. Unterschiede mit der eigenen Patientenpopulation:

- Altersmedian in beiden Studien: 66 Jahre;

- $78 \%$ Raucher in beiden Studien;

- In beiden Studien hatten über 70\% der Studienteilnehmer einen niedrigen Bildungsabschluss (eigene Studie: $72 \%$, italienische Studie $81 \%$ );

- Der Anteil der Frauen (30\%) liegt in der eigenen Studie höher, als es die italienische Studie erwarten ließ. Von den 1277 Patienten waren dort nur 181 (14\%) weiblich.

Zur Epidemiologie des Bronchialkarzinoms schreibt Macha, dass ca. 16\% aller Männer und 9\% aller Frauen, die rauchen, an einem Bronchialkarzinom erkranken. Gleichzeitig seien aber 10$15 \%$ aller Patienten mit Bronchialkarzinom Nichtraucher (Macha 2003). In der eigenen Studie liegt der Nichtraucheranteil mit 22\% etwas höher.

Die verglichenen Daten zeigen, dass die eigene Patientenpopulation ein vergleichbares soziodemographisches Profil wie eine große Gruppe von Bronchialkarzinompatienten eines italienischen Krankenhauses hat und sich somit gut als exemplarisch untersuchte Population eignet. Auf Grund der geringen Patientenanzahl lassen die eigenen Daten aber keinen Rückschluss auf die Allgemeinheit der Bronchialkarzinompatienten zu. 


\subsubsection{Aufklärungsgespräch}

Die Angaben zur Gesprächsdauer des Aufklärungsgesprächs variieren nach Angaben der Patienten in den Jahren 2003-2005 zwischen 2 und 100 Minuten. In Abbildung 3.1.4 ist der jeweilige Median der Gesprächsdauer für jedes Jahr gesondert aufgetragen. Dabei fällt auf, dass die Dauer des Gesprächs aus Sicht der Patienten abnimmt: von einem Median von 30 Minuten im Jahr 2003 auf einen Median von 20 Minuten im Jahr 2005. Im Jahr 2004 errechnet sich sogar nur ein Median von 15 Minuten. Die Berechnung des Medians wurde gewählt, damit zeitliche „Ausreißer“ wenig in die Berechnung eingehen. Diese Zeiten sind subjektive Angaben, da die Dauer der Gespräche nicht mit einer Uhr gemessen wurde und die Zeitangaben einer retrospektiven Befragung entstammen.

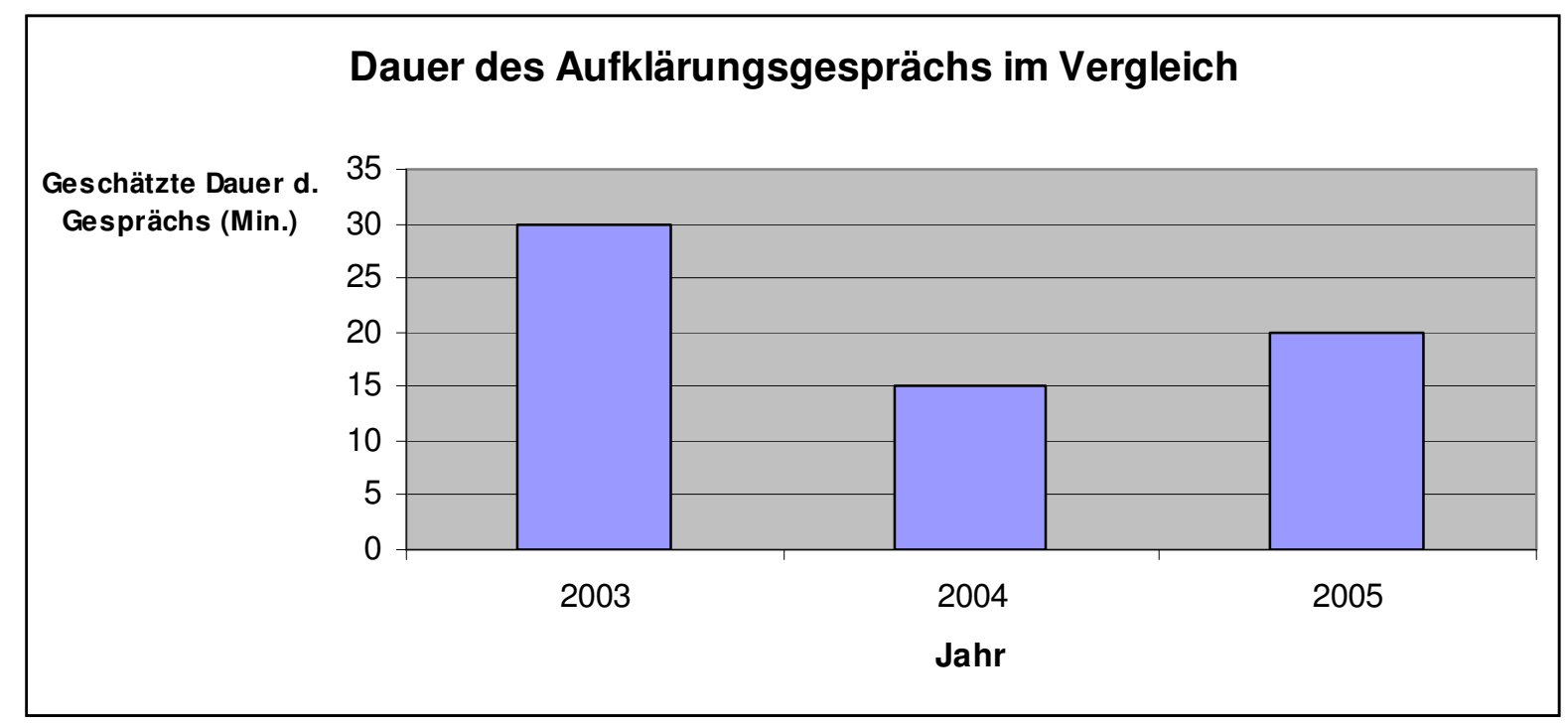

Abbildung 3.1.4. Mediane der von den Patienten geschätzten Dauer des Aufklärungsgesprächs im Vergleich 2003-2005

\subsubsection{Verständlichkeit des Aufklärungsgesprächs}

Als nächster Punkt folgt die Bewertung der Verständlichkeit des Aufklärungsgesprächs aus Sicht der Patienten (s. Kap. 2.2.2.). Diese Bewertung wurde auf einer Likert-Skala mit fünf Abstufungen vorgenommen. Die Patienten konnten angeben ,alles“ bis „gar nichts“ verstanden 
zu haben. Um die Daten vergleichend auswerten zu können, wurde „alles“ verstanden zu haben mit 1 codiert und „gar nichts“ mit 5 (,,wenig“ $=4$; ,,die Hälfte“ $=3$; ,,vieles“ $=2$. In der folgenden Tabelle sind die Mittelwerte der Jahre 2003-2005 vergleichend dargestellt:

\begin{tabular}{|l|l|l|l|}
\hline Jahr & $\mathbf{2 0 0 3}$ & $\mathbf{2 0 0 4}$ & $\mathbf{2 0 0 5}$ \\
\hline Mittelwert & 1,83 & 2,0 & 1,81 \\
\hline
\end{tabular}

Tabelle 3.1.4.1a Mittelwerte der Verständlichkeit des Aufklärungsgesprächs 2003-2005 aus Patientensicht

Der beste Mittelwert wird 2005 mit 1,81 ermittelt, im Jahr 2003 liegt der Mittelwert bei 1,83 und im Jahr 2004 bei 2,0. Von der überwiegenden Zahl der Befragten wird in allen drei Jahren angegeben, dass sie „,vieles“ oder ,alles“ vom Gespräch verstanden haben. Trotz einer von den Patienten angegebenen Abnahme der Gesprächsdauer im Vergleich zu 2003 zeigen die Mittelwerte zur Verständlichkeit keine Verschlechterung.

Die befragten Patienten hatten außerdem die Möglichkeit, Verbesserungsvorschläge für das Aufklärungsgespräch frei $\mathrm{zu}$ formulieren. Diese Vorschläge werden nun im Folgenden vorgestellt. Um die Vorschläge übersichtlich zu gliedern, wurden sie in folgende Kategorien eingeteilt:

- Zeitaspekt

- Gesprächsart

- andere Verbesserungsvorschläge.

\section{Zeitaspekt}

Der Zeitaspekt wurde in allen drei Jahren von insgesamt acht Patienten als verbesserungswürdig angesehen. Dreimal wurde angegeben, dass sich der Arzt mehr Zeit nehmen solle (2003; 2004; $2005)^{8}$. Auch die Verständlichkeit wird als verbesserungswürdig angesehen. So schreibt ein Patient: „vor allem [soll der Arzt] deutsch sprechen, damit es auch jeder versteht“ (2005).

\footnotetext{
${ }^{8}$ Die in Klammern stehenden Jahreszahlen geben das Jahr wieder, auf das sich die Aussage bezieht - also das Jahr des stationären Aufenthalts.
} 


\section{DARSTELLUNG DER UNTERSUCHUNGSERGEBNISSE}

Anhand der Verbesserungsvorschläge lässt sich kein Unterschied zwischen der Zeit vor und während der DRG-Einführung feststellen.

\section{Gesprächsart}

Zur Verbesserung der Gesprächsart geben die Patienten im Jahr 2004 dreimal an, dass weniger Fachausdrücke verwendet werden sollten. Ein Patient schreibt: „Nicht so viele Fachausdrücke verwenden, „Tacheles“ reden.“ (2005). Die Patienten möchten ein offenes Gespräch (2004) und „keine ausweichenden, sondern konkrete Antworten“ (2003). Auch die Prognose sollte in diesem Gespräch mitgeteilt werden (2004). Im Jahr 2003 wünscht ein Patient, dass sich der operierende Arzt bei ihm vorstellt. Ein anderer Patient möchte mehr Informationen über die Zeit nach der Operation und deren Auswirkungen auf den gesamten Körper (2005). Außerdem sei das Eingehen des Arztes auf den jeweiligen Patienten verbesserungswürdig, da sich Patienten allein gelassen fühlten und es schwer sei, mit der Krankheit umzugehen und sie anzunehmen (2004).

Die Verbesserungsvorschläge zur Art des Aufklärungsgesprächs lassen in den drei Jahren keine Unterschiede erkennen. Wichtig scheint den Patienten, dass ein Aufklärungsgespräch ein ehrliches und in verständlichen Worten geführtes Gespräch ist.

\section{Andere Verbesserungsvorschläge}

Neben den oben aufgeführten Verbesserungsvorschlägen zum Zeitaspekt und der Gesprächsart werden noch folgende Vorschläge gemacht: „Gespräche sollten auch innerhalb der Familie geführt werden, damit diese sich besser auf das Krankheitsbild einstellen können“ (2004). Ein anderer Patient schlägt vor, dass es während der Chemotherapie öfter als alle drei Monate Besprechungen geben sollte (2004). Im Jahr 2003 äußert ein Patient, ,es sollte nicht ein, sondern mehrere Gespräche geben, da sich erst im Nachhinein Fragen ergeben“.

Für die Patienten aller drei Jahre ist das Einbeziehen von Angehörigen wichtig. Außerdem werden Folgegespräche nach dem eigentlichen Aufklärungsgespräch gewünscht.

Zum Aufklärungsgespräch kritisch anzumerken ist, dass einige Patienten in jedem Jahr angeben, von ihrem Arzt nicht über verschiedene Behandlungsmöglichkeiten bzw. Nebenwirkungen der Behandlung aufgeklärt worden zu sein. Tabelle 3.1.4.1b gibt einen Überblick über die Anzahl dieser Patienten. 


\section{DARSTELLUNG DER UNTERSUCHUNGSERGEBNISSE}

\begin{tabular}{|l|l|l|l|}
\hline Jahr & $\mathbf{2 0 0 3}$ & $\mathbf{2 0 0 4}$ & $\mathbf{2 0 0 5}$ \\
\hline $\begin{array}{l}\text { Keine Aufklärung über } \\
\text { Nebenwirkungen } \\
\text { (Personenzahl) }\end{array}$ & 3 & 3 & 2 \\
\hline $\begin{array}{l}\text { Keine Aufklärung über } \\
\text { Wirkungsweise } \\
\text { (Personenzahl) }\end{array}$ & 0 & 4 & 2 \\
\hline
\end{tabular}

Tabelle 3.1.4.1b Fehlende Erinnerung an eine Aufklärung über Behandlungsweise bzw. Nebenwirkungen (2003-2005)

\subsubsection{Qualität der ärztlichen Betreuung}

Zur Beurteilung der Qualität der ärztlichen Behandlung werden den Studienteilnehmern folgende Fragen gestellt:

1. Hatten Sie den Eindruck, dass der behandelnde Arzt auf Ihre Wünsche und Bedürfnisse eingegangen ist?

2. Wie beurteilen Sie die ärztliche Behandlung?

Bewertet wird jeweils auf einer Likert-Skala von 1 (,sehr“ bzw. „sehr gut“) bis 5 (,,überhaupt nicht“ / „ungenügend“) (s. Kap. 2.2.2.). In den Jahren 2003 und 2005 errechnet sich ein Mittelwert für die Angaben zum „Eingehen des Arztes auf die Wünsche der Patienten“ von jeweils 1,8. Im Jahr 2004 liegt der Mittelwert mit 1,9 etwas höher und zeigt somit eine etwas schlechtere Beurteilung. Die Patienten beurteilen die ärztliche Behandlung in den Jahren 2003 und $2004 \mathrm{im}$ Mittel mit der Note 2. 2005 wird mit 1,8 die beste Note erreicht. Die Beurteilung der Studienteilnehmer zeigt, dass sich für diese Patienten die Behandlungsqualität nicht verschlechtert, sondern sogar leicht verbessert hat. Tabelle 3.1.5 zeigt den Vergleich der errechneten Mittelwerte für die drei Jahre. 
DARSTELLUNG DER UNTERSUCHUNGSERGEBNISSE

\begin{tabular}{|l|l|l|l|}
\hline Jahr & $\mathbf{2 0 0 3}$ & $\mathbf{2 0 0 4}$ & $\mathbf{2 0 0 5}$ \\
\hline $\begin{array}{l}\text { Mittelwert der Noten für } \\
\text { Eingehen des Arztes auf } \\
\text { Wünsche und } \\
\text { Bedürfnisse }\end{array}$ & 1,8 & 1,9 & 1,8 \\
\hline $\begin{array}{l}\text { Mittelwert der Noten für } \\
\text { ärztliche Behandlung }\end{array}$ & 2,0 & 2,0 & 1,8 \\
\hline
\end{tabular}

Tabelle 3.1.5 Vergleich der Bewertung der ärztlichen Betreuung durch die Patienten (2003-2005)

\subsubsection{Qualität der pflegerischen Betreuung}

Neben der Bewertung der ärztlichen Betreuung wird von den Patienten auch die Betreuung durch die Pflegekräfte beurteilt. Die Mittelwerte für die einzelnen Jahre sind in Tabelle 3.1.6 zusammengestellt. Die Bewertung wird wieder mit Hilfe einer Likert-Skala mit fünf Abstufungen durchgeführt, wobei 1 „sehr gut“ und 5 „,ungenügend“ entspricht. Die Mittelwerte liegen in den Jahren 2003 und 2004 jeweils bei 1,9, im Jahr 2005 wird die Betreuung mit dem Mittelwert 2,0 etwas schlechter bewertet.

\begin{tabular}{|l|l|l|l|}
\hline Jahr & $\mathbf{2 0 0 3}$ & $\mathbf{2 0 0 4}$ & $\mathbf{2 0 0 5}$ \\
\hline $\begin{array}{l}\text { Mittelwert Noten für } \\
\text { pflegerische Betreuung }\end{array}$ & 1,9 & 1,9 & 2,0 \\
\hline
\end{tabular}

Tabelle 3.1.6 Vergleich der Bewertung der pflegerischen Betreuung (2003-2005)

Damit ergibt sich nur ein minimaler Unterschied in der Bewertung der einzelnen Jahre. Vergleicht man diese Werte mit denen der ärztlichen Betreuung, fällt die Bewertung der Ärzteschaft etwas positiver aus.

Der vor der DRG-Einführung befürchtete Qualitätseinbruch der stationären Behandlung lässt sich mit den gewonnen Daten nicht bestätigen.

\subsubsection{Sorgen und Ängste der Patienten}

Die folgenden zwei Punkte der Patientenbefragung befassen sich mit den Sorgen und Ängsten der Patienten. Mit Hilfe des Fragebogens wird evaluiert, welche Sorgen und Ängste Patienten mit einem Bronchialkarzinom haben. Dabei besteht sowohl die Möglichkeit, vorgegebene 


\section{DARSTELLUNG DER UNTERSUCHUNGSERGEBNISSE}

Antwortmöglichkeiten anzukreuzen, als auch freie Anmerkungen $\mathrm{zu}$ machen. In jedem Jahr geben 23-35\% der Studienteilnehmer an, keine Sorgen zu haben. Die meisten Sorgen machten sich die Patienten wegen der zusätzlichen Belastung der Angehörigen. Im Jahr 2003 waren es 32\%, im Jahr 2004 43\% und im Jahr 2005 48\%. Tabelle 3.1.7 und Abbildung 3.1.7a zeigen die Verteilung der angegebenen Ängste und Sorgen im Vergleich der drei Jahre.

\begin{tabular}{|l|l|l|l|}
\hline $\begin{array}{l}\text { Jahr/ } \\
\text { Art der Sorgen }\end{array}$ & $\mathbf{2 0 0 3}$ & $\mathbf{2 0 0 4}$ & $\mathbf{2 0 0 5}$ \\
\hline $\begin{array}{l}\text { Zusätzliche Belastung von } \\
\text { Angehörigen }\end{array}$ & $32 \%$ (13 Patienten) & $43 \%$ (16 Patienten) & $48 \%$ (15 Patienten) \\
\hline Einsamkeit & $5 \%$ (2 Patienten) & $11 \%$ (4 Patienten) & $16 \%$ (5 Patienten) \\
\hline Versorgung zu Hause & $17 \%$ (7 Patienten) & $14 \%$ (5 Patienten) & $19 \%$ (6 Patienten) \\
\hline Keine Sorgen & $27 \%$ (11 Patienten) & $35 \%$ (13 Patienten) & $23 \%$ (7 Patienten) \\
\hline
\end{tabular}

Tabelle 3.1.7 Angaben über Sorgen und Ängste der Patienten 2003-2005 im Vergleich

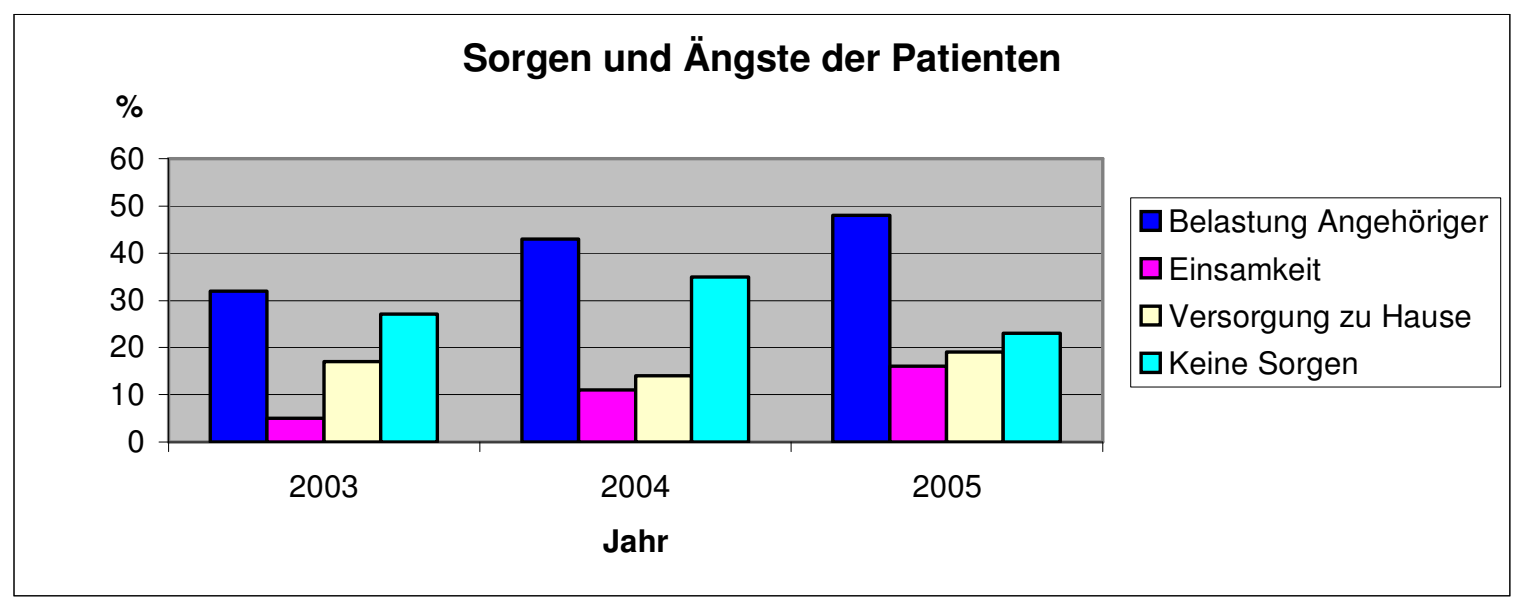

Abbildung 3.1.7a Angaben über Sorgen und Ängste der Patienten 2003-2005 im Vergleich

Von Jahr zu Jahr befürchten prozentual mehr Patienten, ihre eigenen Angehörigen zu belasten. Dieser Trend ist auch in Bezug auf die Daten in den USA interessant. Dort kam es nach Einführung der DRG zu einem vermehrten Pflegebedarf nach Entlassung aus dem Krankenhaus (s. Kap. 1.4.3.). Dass die Patienten sich Sorgen um die Mehrbelastung ihrer Angehörigen machen, könnte ein Hinweis darauf sein, dass auch in Deutschland der Pflegebedarf zunimmt und dadurch die Angehörigen mehr belastet werden. Neben dem Anteil der Sorge um die 
Angehörigen steigt außerdem die prozentuale Anzahl der Patienten, die sich Sorgen um ihre häusliche Versorgung machen von 17\% im Jahr 2003 auf 19\% im Jahr 2005.

Dazu passen auch die von den Patienten selber formulierten Sorgen, die in folgende Bereiche gegliedert werden können

- Familie

- Psyche

- Körperliches Befinden

- Arbeitsplatz und Finanzen.

Diese Art der Darstellung soll die selber formulierten Ängste und Sorgen der Patienten aufzeigen. Es wird kein Vergleich dieser Angaben für die Jahre 2003 bis 2005 durchgeführt, da sich die Patienten nicht in jedem Jahr zu den jeweiligen Bereichen geäußert haben. Die Darstellung dient vielmehr dazu, die Komplexität der Sorgen und Ängste dieser Patientengruppe zu verdeutlichen.

\section{$\underline{\text { Familie }}$}

Die Sorgen um die eigene Familie werden von den Studienteilnehmern auch im Freitext formuliert. So gibt ein Familienvater an, sich Sorgen um seine Frau und seine Tochter zu machen $(2003)^{9}$. Im Jahr 2004 berichtet eine Frau, dass sie sich Sorgen macht, da ihr Ehemann sich nicht mit der Situation auseinandersetzt. Eine andere schreibt, dass sie Angst habe, ihre Tochter zu überlasten (2004). Ein Mann gibt an, sich um seinen Arbeitsplatz, die Kinder und seine Ehefrau zu sorgen (2005).

\section{$\underline{\text { Psyche }}$}

Probleme, die die psychische Verfassung betreffen, werden in den Jahren 2003 und 2004 angegeben. Ein Studienteilnehmer stellt die Frage: „Was soll werden? Der Tod?“ (2003). Im Jahr 2004 hat ein Patient Angst vor der eigenen seelischen Belastung, ein anderer gibt an, psychische Probleme zu haben, da er sich nun schon zum zweiten Mal mit der Diagnose „Krebs“

\footnotetext{
${ }^{9}$ Die in Klammern stehenden Jahreszahlen geben das Jahr wieder, auf das sich die Aussage bezieht - also das Jahr des stationären Aufenthalts.
} 


\section{DARSTELLUNG DER UNTERSUCHUNGSERGEBNISSE}

auseinandersetzen muss (2004). Diese Antworten machen deutlich, dass eine Mitbetreuung der Patienten durch Psychologen und Seelsorger wichtig ist.

\section{Körperliches Befinden}

Auch um ihr körperliches Befinden sorgen sich die Studienteilnehmer. Ein Patient fragt sich, ob er seine frühere Mobilität wieder erlangen kann (2004). Ein anderer sorgt sich, ob er mit der (operierten) Lunge so weiterleben kann (2004).

\section{$\underline{\text { Arbeitsplatz und Finanzen }}$}

Ein weiterer Aspekt, um den sich die Patienten sorgen, ist der Arbeitsplatz. Im Jahr 2003 macht sich ein Studienteilnehmer Sorgen um die Konsequenzen, die durch den Wegfall seiner Arbeitskraft entstehen. Ein anderer schreibt: „Man wird ständig an den Tumor erinnert, es entstehen Belastungen durch postoperative Nachwirkungen, und es kommt $\mathrm{zu}$ finanziellen Einbußen während der Krankheit“"(2005).

\section{Krankheit und Krankheitsverlauf}

Mit dem Krankheitsverlauf beschäftigen sich viele Ängste und Sorgen der Patienten. Im Jahr 2003 schreibt ein Studienteilnehmer, Angst vor dem weiteren Verlauf der Krankheit zu haben. Ein anderer fragt: „Wie entwickelt sich die Krankheit weiter? Werde ich wieder ganz gesund?“ (2003). 2004 sorgt sich ein Patient, was ihn erwartet und was er tun kann und sollte. „Ich habe Angst vor der Ungewissheit über den zukünftigen Krankheitsverlauf" schreibt ein Patient 2005.

Diese Kommentare zeigen, dass sich die befragten Patienten Sorgen um das Thema Gesundheit machen. Aber auch um die Arbeitsstelle und das eigene körperliche Befinden sorgen sich die Studienteilnehmer. Diese Sorgen und Ängste müssen, um eine gute Behandlungsqualität zu gewährleisten, angesprochen und ernst genommen werden. Deshalb beschäftigt sich die nächste Frage mit den Gesprächspartnern, mit denen die Patienten über ihre Ängste und Sorgen sprechen können. 77 Patienten von 109 Befragten (71\%) geben insgesamt in allen drei Jahren an, mit ihren Angehörigen über Sorgen und Ängste reden zu können. 69 Patienten (63\%) geben den Klinikarzt als Gesprächspartner an. Eine Berechnung der Prozentzahlen für die einzelnen Jahre zeigt, dass im Jahr 2003 66\%, im Jahr 2004 60\% und im Jahr 2005 65\% der Patienten angeben, mit ihrem 
behandelnden Klinikarzt über ihre Ängste und Sorgen sprechen zu können. Abbildung 3.1.7b vergleicht diese prozentuale Verteilung in jedem Jahr miteinander.

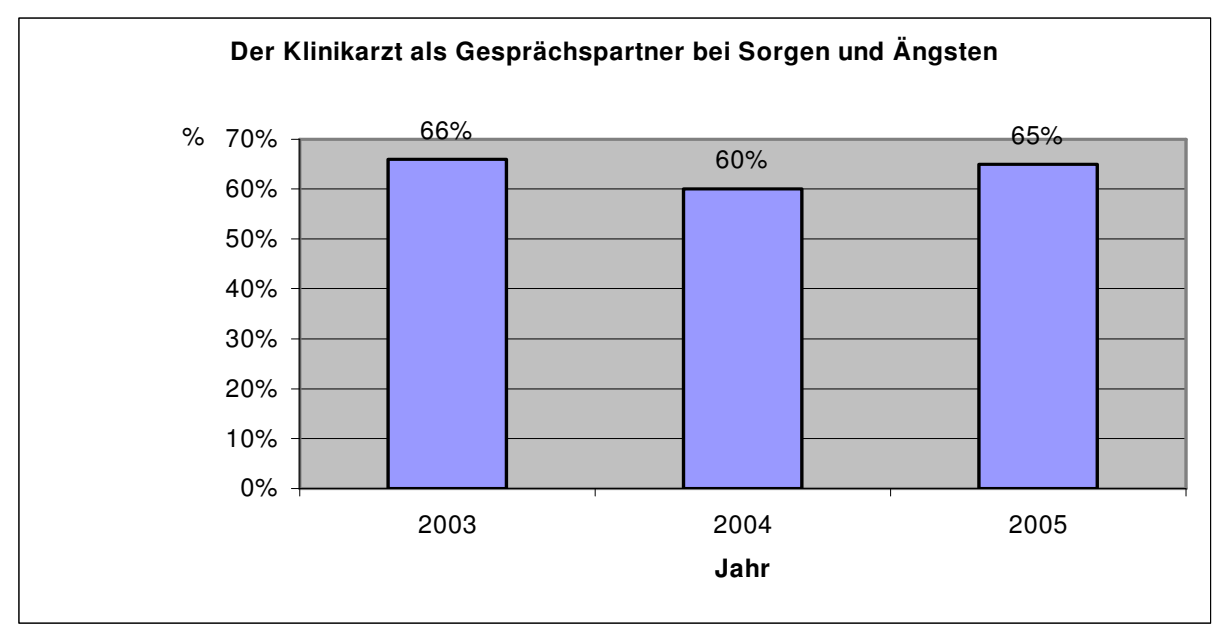

Abbildung 3.1.7b Häufigkeit der Patientenangaben über den Klinikarzt als Ansprechpartner bei Sorgen und Ängsten im Vergleich 2003-2005

In jedem Jahr antworten mindestens $60 \%$ der Studienteilnehmer, ihren Klinikarzt als Ansprechpartner für Sorgen und Ängste zu haben. Dieses Ergebnis unterstreicht die wichtige Rolle, die der Arzt für die psychische Betreuung hat. Wie bereits erwähnt, ist es für eine gute Behandlungsqualität wichtig, auch die Rolle des Arztes als Gesprächspartner der Patienten zu berücksichtigen und im DRG-System abzubilden.

\subsubsection{Pflegebedarf bei Entlassung}

Die nächste Frage bezieht sich auf den Pflegebedarf der Patienten bei Entlassung aus dem Krankenhaus. Es wird gefragt, ob und wenn ja welche Art der Pflege und Betreuung die Patienten in Anspruch nehmen. Wie bereits in der Einleitung erwähnt (s. Kap. 1.4.3.), hat der Pflegebedarf zum Zeitpunkt der Entlassung nach DRG-Einführung in den USA signifikant zugenommen.

In der vorliegenden Studie geben im Jahr 200319 Patienten (46\%) an, keine Pflege zu benötigen, 2004 sind es 20 Patienten (54\%) und 200517 Patienten (55\%). Für die untersuchte Patientenpopulation lässt sich somit keine Erhöhung, sondern eine leichte Minderung des 
Pflegebedarfs bei Entlassung verzeichnen. Die Patienten, die Pflege benötigen, werden zu mindestens einem Drittel bis fast der Hälfte in den Jahren 2003 bis 2005 von ihrer Familie oder den Angehörigen versorgt bzw. gepflegt. Die Pflege durch Freunde, ambulante Dienste und Kurzzeitpflege wurde seltener benötigt. Die folgende Tabelle vergleicht die Angaben zum Pflegebedarf bei Entlassung in den untersuchten Jahren.

\begin{tabular}{|l|ll|lllll|}
\hline Jahr & \multicolumn{2}{|l|}{$\mathbf{2 0 0 3}$} & $\mathbf{2 0 0 4}$ & $\mathbf{2 0 0 5}$ & \\
\hline Keine Pflege & $46 \%$ & (19 Patienten) & $54 \%$ & (20 Patienten) & $55 \%$ & (17 Patienten) \\
\hline Familie & $49 \%$ & (20 Patienten) & $35 \%$ & (13 Patienten) & $42 \%$ & (13 Patienten) \\
\hline Freunde & $5 \%$ & (2 Patienten) & $3 \%$ & (1 Patient) & $7 \%$ & (2 Patienten) \\
\hline Ambulante Pflege & $2 \%$ & (1 Patient) & $5 \%$ & (2 Patienten) & $3 \%$ & (1 Patient) \\
\hline Kurzzeitpflege & $2 \%$ & (1 Patient) & $3 \%$ & (1 Patient) & $3 \%$ & (1 Patient) \\
\hline $\begin{array}{l}\text { Dauerhafte Unter- } \\
\text { bringung in einer } \\
\text { Pflegeinstitution }\end{array}$ & 0 & & 0 & & 0 & & \\
\hline
\end{tabular}

Tabelle 3.1.8 Pflegebedarf der Patienten bei Entlassung im Vergleich 2003-2005

Diese Tabelle verdeutlicht, dass der Pflegebedarf der befragten Patientenpopulation nach Entlassung während der DRG-Einführung nicht zugenommen, sondern im Gegenteil abgenommen hat. Dies ist ein nicht zu erwartendes Ergebnis, da die Studien aus den USA, wie oben erwähnt, einen signifikanten Anstieg des Pflegebedarfs bei Entlassung vorausgesagt haben. Gleichzeitig wird aus den eigenen Daten ersichtlich, dass der größte Teil der Pflege und Betreuung der untersuchten Patienten von den Angehörigen geleistet wird. Bei der Deutung dieser Daten muss aber eine mögliche Verzerrung der Untersuchungsergebnisse berücksichtigt werden, denn es bleibt unklar, ob der Fragebogen die Patienten in einer dauerhaften Pflegeeinrichtung überhaupt erreicht hat. Darüber können in dieser Studie keine Angaben gemacht werden. 


\subsubsection{Anfahrtsweg zur behandelnden Institution}

Der nun folgende Absatz beschäftigt sich mit der Länge des Anfahrtswegs zum Behandlungsort. In der Abbildung 3.1.9 sind die durchschnittliche Länge des tatsächlichen Anfahrtswegs und die Länge des Anfahrtswegs aufgezeichnet, die die Patienten in ihrem Gesundheitszustand für angemessen halten. Der Median der Länge des durchschnittlich tatsächlich zurückgelegten Anfahrtsweges liegt zwischen 35 bis 40 km. Im Jahr 2003 und 2005 errechnet sich ein Median von $35 \mathrm{~km}$, im Jahr 2004 ein Median von $40 \mathrm{~km}$. Bei der Beurteilung der Länge des Anfahrtsweges sollte berücksichtigt werde, dass es sich um zum Teil schwerkranke Patienten handelt, für die auch schon kürzere Wegstrecken eine gesundheitliche Belastung bedeuten.

Aus den US-amerikanischen Studien geht hervor, dass sich die medizinische Infrastruktur nach DRG-Einführung geändert hat. Auch für Deutschland wird eine solche Veränderung von Unternehmensberatungen prognostiziert (Hehner et al. 2002). Aus den eigenen Daten ist eine solche Veränderung nicht ersichtlich. Es kann allerdings angenommen werden, dass sich die Auswirkungen der DRG-Einführung in Bezug auf die medizinische Infrastruktur erst längerfristig zeigen werden, denn zum Zeitpunkt der Befragung hatte die Einführung der DRG erst begonnen, und eine Schließung von Kliniken und somit eine eventuelle Verlängerung des Anfahrtswegs werden sich erst in den kommenden Jahren zeigen.

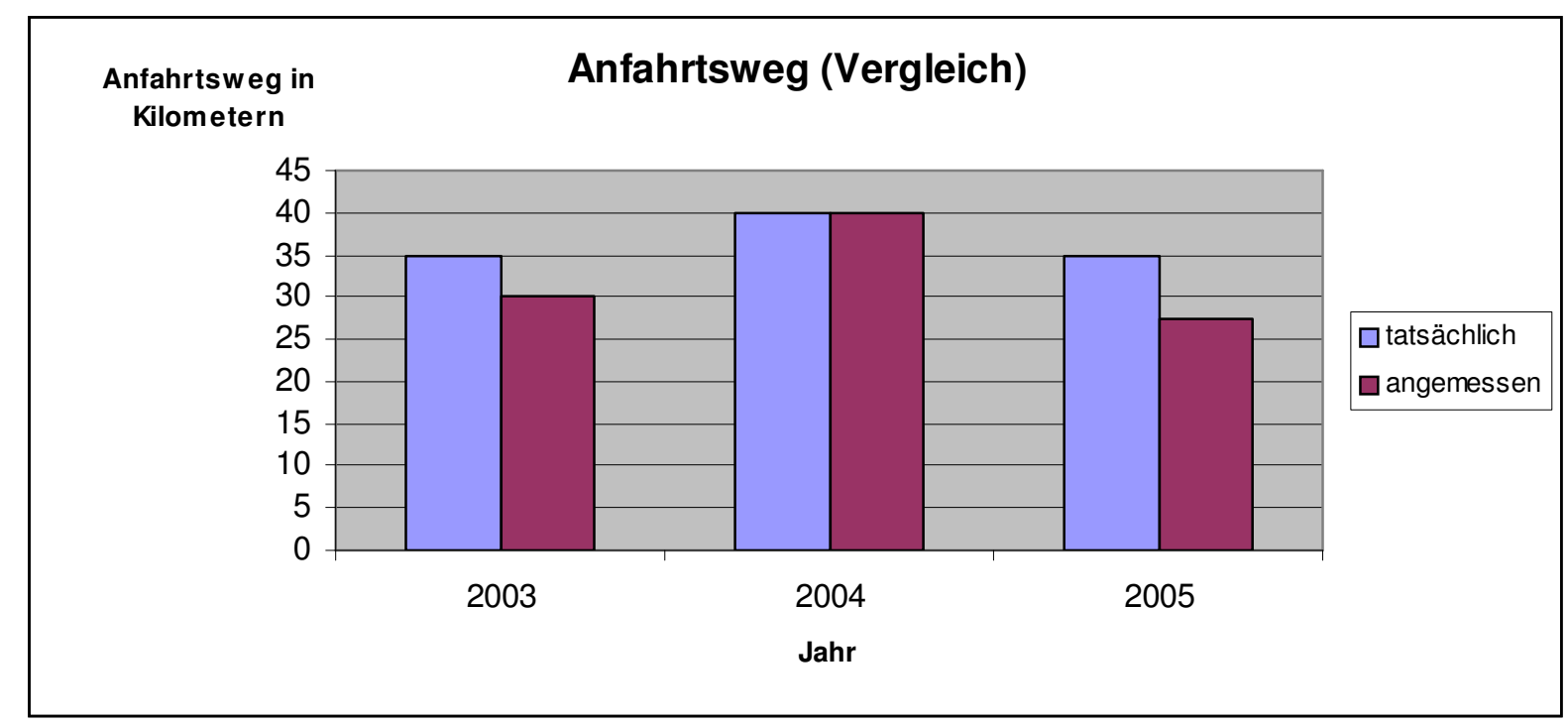

Abbildung 3.1.9 Vergleich der Mediane der Länge der tatsächlichen Anfahrtswege und der ,angemessenen““ Anfahrtswege aus Sicht der Patienten 2003-2005 


\section{DARSTELLUNG DER UNTERSUCHUNGSERGEBNISSE}

Die Patienten wurden außerdem zu der Wegstrecke befragt, die sie in ihrem Gesundheitszustand für angemessen hielten. Die Mediane dieser Werte lagen in den Jahren 2003 und 2005 unter denen des „tatsächlichen“ Wertes. Im Jahr 2004 entsprach der Median des tatsächlichen Weges, dem der von den Patienten für angemessen gehalten wurde.

Festzustellen ist außerdem, dass sich die als angemessen empfundene Strecke häufig an der Länge der tatsächlichen Strecke orientiert. 34 von 109 Patienten geben exakt die gleiche Länge des als angemessenen empfundenen und des tatsächlich zurückgelegten Weges an. Bei weiteren neun Patienten beträgt die Differenz von angemessen empfundenem und tatsächlichem Anfahrtsweg 10 oder weniger Kilometer. Daraus kann geschlossen werden, dass die Gewöhnung bei der Beurteilung der Angemessenheit eine große Rolle spielt.

Die Frage zur Länge des angemessenen Anfahrtsweges wurde in insgesamt 28 der ausgewerteten Fragebögen nicht beantwortet.

\subsubsection{Lebensqualität zum Zeitpunkt der Befragung}

Die Beurteilung der Lebensqualität der Patienten zum Zeitpunkt der Befragung soll Aufschlüsse über die vermuteten Veränderungen der Behandlungsqualität geben. Die Lebensqualität stellt dabei ein Messkriterium dar, das in allen Jahren miteinander verglichen wird. Würde sich die Lebensqualität der behandelten Patienten nach DRG-Einführung verschlechtern, würde diese Verschlechterung im Widerspruch zum Nichtschädigungsprinzip stehen. Die Patientenbefragung fand jeweils in den Monaten Februar bis Mai statt. Die Patienten wurden im jeweils vorangegangenen Jahr in einer der teilnehmenden Kliniken behandelt. Die Angaben zur Lebensqualität beziehen sich nicht auf den Zeitpunkt nach Entlassung, sondern auf den Zeitpunkt der Befragung einige Monate nach Entlassung aus der stationären Behandlung. Da diese Zeitspanne in den Jahren 2004-2006 gleich lang ist, können die Daten trotzdem als Messkriterium herangezogen werden. 


\section{DARSTELLUNG DER UNTERSUCHUNGSERGEBNISSE}

\begin{tabular}{|l|l|l|l|}
\hline \multicolumn{1}{|c|}{ Jahr } & $\mathbf{2 0 0 3}$ & $\mathbf{2 0 0 4}$ & $\mathbf{2 0 0 5}$ \\
\hline Funktionsskalen & & & \\
\hline Körperliche Funktion & 56 & 57 & 59 \\
\hline Rollenfunktion $^{10}$ & 46 & 40 & 46 \\
\hline Seelische Funktion & 49 & 51 & 53 \\
\hline Kognitive Funktion $^{\prime}$ & 67 & 69 & 78 \\
\hline Soziale Funktion & 51 & 55 & 48 \\
\hline Lebensqualität $^{\text {SI }}$ & 46 & 47 & 48 \\
\hline
\end{tabular}

\begin{tabular}{|l|l|l|l|}
\hline Symptomskalen & & & \\
\hline Müdigkeit & 58 & 56 & 50 \\
\hline Übelkeit und Erbrechen & 16 & 20 & 19 \\
\hline Schmerzen & 46 & 47 & 41 \\
\hline Dyspnoe & 60 & 67 & 66 \\
\hline Schlafstörungen & 42 & 54 & 37 \\
\hline Appetitlosigkeit & 29 & 31 & 26 \\
\hline Obstipation & 22 & 28 & 14 \\
\hline Diarrhoe & 15 & 16 & 18 \\
\hline Finanzielle Beeinträchtigung & 34 & 32 & 41 \\
\hline
\end{tabular}

Tabelle 3.1.10a Auswertung der Fragebögen zur Lebensqualität, transformierte Daten aller drei Jahre im Vergleich

Tabelle 3.1.10a vergleicht die Mittelwerte aller Funktions- und Symptomskalen der einzelnen Jahre miteinander. Die Mittelwerte befinden sich durch eine Transkription der Rohwerte (s. Kap. 2.2.2.) jeweils auf einer Skala von 0-100. Hohe Werte in den Funktionsskalen bedeuten einen guten Funktionszustand / Lebensqualität, hohe Werte in den Symptomskalen bedeuten eine starke Ausprägung des jeweiligen Symptoms.

\footnotetext{
${ }^{10}$ Die „Rollenfunktion“ entspricht der Funktion, die die Person in ihrer Arbeits- und Freizeitwelt ausführt.

${ }^{11}$ Die „Soziale Funktion“ entspricht der Funktion, die die Person im Umgang mit den Mitmenschen erfüllt (s. 2.2.2).
} 


\section{DARSTELLUNG DER UNTERSUCHUNGSERGEBNISSE}

Um die Funktions- und Symptomwerte der einzelnen Jahre besser vergleichen zu können, werden die Mittelwerte der obigen Tabelle (ungerundete Zahlen) jeweils summiert und durch die Anzahl der Skalen geteilt. So erhält man folgende Mittelwerte:

\begin{tabular}{|l|l|l|l|}
\hline & $\mathbf{2 0 0 3}$ & $\mathbf{2 0 0 4}$ & $\mathbf{2 0 0 5}$ \\
\hline Funktionswerte & 52,3 & 53 & 55,2 \\
\hline Symptomwerte & 35,7 & 39 & 34,5 \\
\hline
\end{tabular}

Tabelle 3.1.10b Vergleich der transformierten Funktions- und Symptommittelwerte von 2003-2005

Die Zahlenwerte dieser Tabelle dienen dem Vergleich der Lebensqualität der drei Jahre. Diese Zahlen lassen keine konkrete Aussage über die Lebensqualität der Befragten zu sondern dienen dazu, mögliche Veränderung in der Beurteilung der Lebensqualität während des Befragungszeitraums sichtbar zu machen. Dabei zeigt sich, dass die Bronchialkarzinompatienten im Jahr 2005 den höchsten Funktionswert, also die beste Funktionsfähigkeit, haben und gleichzeitig den niedrigsten Symptomwert, also auch am wenigsten durch die oben aufgeführten Symptome beeinträchtigt sind.

Die Lebensqualität der Patientenpopulation dieser Studie nimmt im untersuchten Zeitraum nach eigenen Angaben nicht ab. Dies ist ein wichtiges Ergebnis im Hinblick auf das Nichtschädigungsprinzip. Die Befürchtung, dass die Patienten nach DRG-Einführung in schlechterem Gesundheitszustand als vor der Einführung aus der Klinik entlassen werden, lässt sich durch die eigenen Daten nicht bestätigen.

\subsection{Auswertung der Ärzteinterviews}

Im Rahmen der vorliegenden Studie wurden neben der Befragung der Patienten auch persönliche Interviews mit Ärzten durchgeführt. Es wurden zwölf Ärzte aus den drei an der Studie teilnehmenden Häusern (Universitätsklinikum Göttingen, Evangelisches Krankenhaus Weende, Krankenhaus Lenglern) befragt. Die Befragung fand anhand von teilstrukturierten Interviews statt. Die Fragen und die Methodik wurden bereits in Kapitel 2.3. erläutert. Um zu gewährleisten, dass die interviewten Ärzte eine Veränderung seit Einführung der DRG zum Jahresbeginn 2004 beurteilen konnten, sollten sie mindestens seit Anfang 2003 in der jeweiligen Abteilung tätig 
sein. Die Befragten waren sowohl Chef-, als auch Ober- und Assistenzärzte und in unterschiedlichen Abteilungen beschäftigt (s. u.). Die Befragung fand im Zeitraum von November 2005 bis Januar 2006 statt.

\subsubsection{Vorbemerkung}

Im Krankenhaus Lenglern und dem Evangelischen Krankenhaus Weende ist das DRGAbrechnungssystem bereits im Jahr 2003 kostenneutral eingeführt worden. Diese Häuser haben 2003 ihr Abrechnungssystem freiwillig auf DRG umgestellt. Die Vergütung erfolgte aber bis Januar 2004 wie im Universitätsklinikum Göttingen über das alte Abrechnungssystem nach Tagessätzen.

\subsubsection{Auswertung der soziodemographischen Daten der Ärzte}

Alle befragten Ärzte sind männlich. Ihr Alter liegt zwischen 33 und 59 Jahren. Die Ärzte arbeiten zum Zeitpunkt der Befragung zwischen 3 und 13 Jahre (Mittelwert 6 Jahre) in der jeweiligen Abteilung. Die aus dem Universitätsklinikum Göttingen befragten Ärzte arbeiten in der Abteilung für Hämatologie und Onkologie und der Strahlentherapie. Die Ärzte des Evangelischen Krankenhauses Weende arbeiten in der Abteilung für Allgemeinchirurgie. Die Ärzte im Krankenhaus Lenglern sind auf pulmonologischen Stationen tätig. Allen Ärzten gemeinsam ist die Behandlung von Patienten mit Bronchialkarzinomen.

\subsubsection{Angaben zu den Arbeitsbedingungen}

\subsubsection{Veränderungen der Arbeitsbedingungen}

Die ersten beiden Fragen des Fragenkatalogs beziehen sich auf die Veränderungen der Arbeitsbedingungen für Ärzte seit Einführung der DRG im Jahr 2004. Die Ärzte berichten, dass es seit 2004 einen größeren Dokumentationsaufwand gebe (Interviews A1, A6, A7, A10) und die Liegezeiten der Patienten abgenommen habe (A1, A5, A10, A12). Außerdem sei ein Teil der Behandlung von Tumorpatienten (besonders die intravenöse Applikation von Chemotherapien) in den ambulanten Sektor verschoben, so dass die Patienten, die stationär aufgenommen werden, 


\section{DARSTELLUNG DER UNTERSUCHUNGSERGEBNISSE}

subjektiv kränker seien als vor der DRG-Einführung (A2, A4), da diejenigen die sich in einem besseren Gesundheitszustand befänden, nach Möglichkeit ambulant versorgt würden. Ein Arzt der chirurgischen Station im Evangelischen Krankenhaus Weende benutzt den Begriff „Fließbandarbeit“"(A4).

Die befragten Ärzte sind für die Kodierung der DRG mitverantwortlich. Von ärztlicher Seite sind an den teilnehmenden Häusern zudem Kontrollen zur Verbesserung der Kodierung eingebracht worden. So gibt es im Universitätsklinikum in der Abteilung Hämatologie und Onkologie seit dem Jahr 2004 eine Zusatzkraft zur optimierten Kodierung. Im Evangelischen Krankenhaus Weende übernimmt ein Oberarzt die Kontrolle des richtigen Kodierens. Da nur die korrekt kodierten Leistungen vergütet werden, entsteht ein Mehraufwand an Dokumentation, der sich in den Angaben der Ärzte widerspiegelt. Dies ist ein erwarteter Nebeneffekt der DRG-Einführung (s. Kap. 1.5.).

\subsubsection{Aufnahme- und Entlassungskriterien}

Die Aufnahme- und Entlassungskriterien haben sich nach Angaben der Ärzte nicht wesentlich verändert. „Es besteht aber kein Anreiz mehr, Patienten länger als medizinisch notwendig in der Klinik zu lassen“, so ein Oberarzt (A1). Dies könnte ein Vorteil für die Patienten sein, da sie schnell in ihre gewohnte Umgebung zurückkehren können. Ein anderer Arzt beschreibt in Bezug auf Entlassungskriterien, dass im Zweifelsfall im Sinne des Patienten entschieden werde (A7). Er befürchtet aber, dass das Geld in Zukunft eine größere Rolle spielen werde und es dann auch zu früheren Entlassungen in einem noch nicht ausreichenden Gesundheitszustand kommen könnte. Ein Stationsarzt aus dem Krankenhaus Lenglern sagt, dass man sich für Tumorpatienten ausreichend Zeit nehme (A8). Nach Angaben der Ärzte ist es in den an der Studie teilnehmenden Häusern nicht zu den von einigen Medien prophezeiten „blutigen Entlassungen“ gekommen. Aus Sicht der Ärzte ist eine frühzeitige Entlassung der Patienten in gutem Gesundheitszustand sogar förderlich für die Patienten. 


\subsubsection{Kassenpatienten und Selbstzahler}

Die Unterschiede in der Behandlung von Kassenpatienten und Selbstzahlern haben sich während der DRG-Einführung aus Sicht der befragten Ärzte nicht verändert. Acht Ärzte (A1, A3, A5, A8, A9, A10, A11, A12) geben an, dass sie vor wie nach der DRG-Einführung keine Unterschiede in der Behandlung von Kassenpatienten und Selbstzahlern machen. Der einzige Punkt, in dem die Art der Versicherung bei Patienten mit Bronchialkarzinom eine Rolle spiele, sei die PETUntersuchung (Positronen-Emissions-Tomographie), mit der eine sehr sensible Darstellung von Karzinomen in der Lunge möglich ist. Diese werde von den gesetzlichen Krankenkassen nicht bezahlt (A1, A2). Außerdem könne es zu längeren Wartezeiten für Diagnostik und Therapie bei gesetzlich versicherten Patienten kommen (A1).

\subsubsection{Aufklärungsgespräch}

Die Ärzte machen außerdem Angaben zur durchschnittlichen Dauer des ersten Gespräches, in dem einem Patienten die Diagnose Lungenkrebs mitgeteilt wird. Die Zeitangaben der Ärzte variieren dabei zwischen 10 und 60 Minuten.

Im Universitätsklinikum Göttingen werden im Durchschnitt ca. 45 Minuten angegeben. Die Dauer variiert nach Angaben der Ärzte im Universitätsklinikum zwischen 30 und 60 Minuten. Im Krankenhaus Lenglern beträgt die Dauer eines Aufklärungsgesprächs nach Angaben der befragten Ärzte ca. 30 Minuten. Die einzelnen Angaben variieren in dieser Klinik stark. Ein Arzt gibt an, bis zu 60 Minuten zu benötigen (A7), ein anderer gibt einen Zeitraum zwischen 10 und 25 Minuten an. Im Evangelischen Krankenhaus Weende dauert ein Aufklärungsgespräch laut Ärzten zwischen 10 und 30 Minuten. Der errechnete Durchschnittswert des Aufklärungsgesprächs aller befragten Ärzte beträgt 36 Minuten.

Die Angaben der Ärzte sind wie die Angaben der Patienten zur Zeitdauer keine objektiven Daten, sondern erinnerte Werte. Für die vorliegende Studie ist vor allem von Interesse, ob sich die Qualität der Kommunikation von Arzt und Patient verändert. Unter diesem Aspekt sind die folgenden Angaben zu bewerten. 


\section{DARSTELLUNG DER UNTERSUCHUNGSERGEBNISSE}

Ärzte aus allen an der Studie teilnehmenden Häusern geben an, dass die Dauer des Aufklärungsgespräches immer auch vom Patienten abhänge. „Ich nehme mir soviel Zeit, wie der Patient braucht", berichtet ein Arzt aus dem Krankenhaus Lenglern (A8). Ein anderer Arzt aus dem Evangelischem Krankenhaus Weende sagt, dass er mit ca. 10 Minuten zu wenig Zeit für die Gespräche habe und dass er die Patienten nur während der morgendlichen Visite sehe, diese müsse auf seiner chirurgischen Station innerhalb von einer Stunde für 30 Patienten gemacht werden (A11).

Von Seiten der übrigen Ärzte wird betont, dass man sich soviel Zeit für ein solches Gespräch nimmt, wie der jeweilige Patient benötigt (A2, A5, A6, A7, A8). Aus der Sicht der meisten befragten Ärzte besteht auch nach Einführung der DRG die Möglichkeit, die Dauer des Gesprächs vom jeweiligen Patienten abhängig zu machen. Dies ist im Sinne einer guten Qualität des ärztlichen Gesprächs positiv zu werten.

Ein Arzt aus dem Krankenhaus Lenglern gibt an, dass Folgegespräche nach der ersten Diagnosemitteilung, an Lungenkrebs erkrankt zu sein, vorgesehen seien (A5). Ob mit dem Patienten noch weitere Gespräche über die Diagnose geführt würden, hänge vom jeweiligen Patienten ab (A2). Da sich der Zeitdruck für die Ärzte vergrößert habe, sei dies nicht einfach durchzuführen, aber es werde versucht, dem einzelnen Patienten gerecht zu werden (A6). Folgegespräche sollten am besten unter Einbeziehung der Angehörigen geführt werden und einige Tage nach dem ersten Gespräch stattfinden, da sich Fragen oft erst mit der Zeit entwickelten (A8). Ein Arzt aus dem Evangelischen Krankenhaus Weende sagt, dass es von Seiten der Chirurgen keine weiteren Gespräche gebe und er dies als unbefriedigend empfinde (A11).

In dem Aufklärungsgespräch und den eventuell folgenden Gesprächen wird nach Angaben der Ärzte mit den Patienten über alle Behandlungsoptionen gesprochen (A2), wobei bei jüngeren Patienten eher ein kurativer Therapieansatz angestrebt werde, der häufig eine aggressive Therapie erfordere. Auch über palliative Maßnahmen und eine Unterbringung in einem Hospiz werde gegebenenfalls mit dem Patienten gesprochen (A6), denn für einige Patienten sei das Leben ohne Therapie, aber mit palliativen Maßnahmen, eine bessere Art der Versorgung (A9).

Außerdem werde dem Patienten mitgeteilt, welche Therapie aus medizinischer Sicht die sinnvollste sei (A5) bzw. warum von ärztlicher Seite eine bestimmte Vorgehensweise gewählt 
würde (A8). Ein Arzt aus dem Krankenhaus Lenglern sagt zum Aufklärungsgespräch: „Der Patient ist die Hauptperson, es muss versucht werden, ihm alle Informationen zu geben, damit er selber mündig entscheiden kann.“ (A7). Dieses Vorgehen sei aber in der Praxis schwierig, da der Arzt sich nie sicher sein könne, dass der Patient das Wesentliche verstanden habe.

\subsubsection{Leitlinien}

Für die Behandlung von Patienten mit einem Bronchialkarzinom gibt es, wie auch für andere Krebserkrankungen, Leitlinien, die sich nach der genauen Diagnose richten wie z.B. die Leitlinien der Deutschen Krebsgesellschaft oder Studienprotokolle (A3). Die onkologischen Patienten werden in verschiedenen Institutionen behandelt und bei schwierigen Entscheidungen in einer gemeinsamen Tumorkonferenz vorgestellt, in der alle behandelnden Ärzte zusammenkommen (Onkologen, Strahlentherapeuten, Chirurgen, Lungenfachärzte). In dieser Konferenz werde die weitere Vorgehensweise und die Behandlung besprochen. Der Patient bekomme so die nach heutigem Stand der Wissenschaft beste Therapie (A3).

\subsubsection{Individuelle Betreuung}

Die an der vorliegenden Studie teilnehmenden Ärzte werden außerdem befragt, wie sie vor und nach DRG-Einführung auf individuelle Wünsche und Bedürfnisse der Patienten eingehen konnten bzw. können. Für eine individuelle Betreuung ist es aus Sicht der Ärzte wichtig, auch die Familie mit einzubeziehen (A1, A2). Es werde versucht, auf einzelne Wünsche einzugehen, dies sei aber nicht immer möglich (A6). Andere Ärzte geben an, dass es nach wie vor möglich sei, auf individuelle Bedürfnisse einzugehen (A9, A10), es aber schwieriger geworden sei, Patienten über ein Wochenende nach Hause $\mathrm{zu}$ entlassen und in der darauffolgenden Woche wieder aufzunehmen (A2, A3, A7). Diese „Wochenendentlassungen“ hätten abgenommen (A2). Wann ein Patient entlassen werden kann, werde individuell entschieden und mit dem Patienten abgestimmt (A2).

Die Einführung von Fallpauschalen könnte zum „Splitten“ der Behandlung eines Patienten führen. Das bedeutet, dass ein Patient mit der gleichen Diagnose mehrfach aufgenommen und 


\section{DARSTELLUNG DER UNTERSUCHUNGSERGEBNISSE}

wieder entlassen wird, um jeweils das Geld für den Krankenhausaufenthalt zu bekommen. Um dies zu verhindern, wurde im Rahmen der DRG-Einführung ein Sicherungssystem eingebaut, das das „Splitten“ von Patientenaufenthalten nicht erlaubt. Unter diesem Aspekt sind die Aussagen der Ärzte zu den „Wochenendentlassungen“ zu sehen, da bei Kontrollen des Medizinischen Dienstes der Krankenkassen ein sogenanntes Splitting vermutet werden könnte.

\subsubsection{Neue Behandlungsoptionen}

Es gibt seit der DRG Einführung 2004 neue Behandlungsoptionen und neue diagnostische Verfahren für Patienten mit einem Bronchialkarzinom. Als diagnostisches Verfahren ist die PET (Positronen-Emissions-Tomographie) eingeführt worden (s.o.). $\mathrm{Zu}$ diesem neuen diagnostischen Verfahren stellt ein Arzt im Rahmen des Interviews die Frage, ob eine maximale Diagnostik auch wirklich immer jedem Patienten zugute komme (A7). Nach Angaben dieses Arztes sei in Bezug auf die Diagnostik eine sinnvolle Kostenreduktion eingetreten, da nicht jede diagnostische Maßnahme auch eine Konsequenz für die Therapie habe und somit nicht bei jedem Patienten notwendig sei.

An therapeutischen Ansätzen gebe es zusätzlich neue, teurere Medikamente, deren Wirkmechanismus z.B. auf der Unterbrechung von Signaltransduktionswegen beruhe (A2). Diese Medikamente seien aber für alle Patienten zugänglich, wenn es für ihre Therapie sinnvoll sei und sie in die jeweilige Studie eingeschlossen wären.

Veränderungen in den Behandlungsergebnissen geben nur zwei Ärzte an: „Es gibt eventuell eine Lebenszeitverlängerung im Monatsbereich“ (A2), „einige Untergruppen profitieren von Neuerungen“(A10).

Epidemiologische Daten zeigen, dass sich die 5-Jahres-Überlebensrate des Bronchialkarzinoms in den letzten 20 Jahren nur von ca. 11\% auf 15\% verbessert hat. Damit ist die Prognose dieser Krebsart weiterhin schlecht (Macha 2003). 


\subsubsection{Persönliche Beurteilung der DRG-Einführung}

Am Ende des Interviews besteht für die Ärzte die Möglichkeit, ihre persönliche Meinung zur Einführung des DRG-Systems zu äußern.

Sieben von zwölf Ärzten begrüßen die Einführung von Fallpauschalen (A1, A2, A3, A6, A7, A8, A12), unter anderem, weil im Rahmen der Vergütung durch Tagessätze „stationäre Aufenthalte vergütet wurden, ohne dass es dabei Anwendungen für den Patienten gab“(A1). Nun gebe es keinen Anreiz, Patienten unnötig lange im Krankenhaus zu behalten. Dies komme auch den Patienten zugute (A4). Ein anderer positiver Aspekt sei, dass es für das eigene Handeln eine größere Transparenz gebe und der einzelne Arzt mehr Überblick über die Therapiequalität und seine Behandlungserfolge bekomme (A7). Auch für epidemiologische Studien sei dieses System gut, denn durch die stärkere Transparenz würden mehr Daten öffentlich gemacht (A8). Außerdem sei eine Umverteilung der noch vorhandenen Gelder nötig, um die medizinischen Ressourcen möglichst gerecht zu verteilen (A11).

Ein Arzt gibt an, anfänglich von der Idee einer fallpauschalisierten Abrechnung begeistert gewesen zu sein, weil er dachte, die Vergütung sei mehr auf den einzelnen Patienten zugeschnitten. In der Praxis sehe er nun aber, dass das korrekte Kodieren sehr viel Zeit in Anspruch nehme und es durch eine schlechte Abbildung einzelner Fälle zu unlogischen Entgelten komme (A6).

Die Gefahr des neuen Systems sieht einer der befragten Ärzte darin, dass sich mit der Zeit der Billigste und nicht der qualitativ Beste durchsetzt (A1). Deshalb sei ein gutes Qualitätsmonitoring unabdingbar. Nach der DRG-Einführung werde die Palliativmedizin zu schlecht vergütet und chronisch Kranke ließen sich schlecht in diesem System abbilden, warnt ein anderer Arzt (A2). Außerdem befürchten die Ärzte, dass die soziale Komponente vernachlässigt werde (A4) und die Zeit für den Kontakt und die Gespräche mit den Patienten zu kurz komme (A11). Durch den erhöhten „Umsatz“ an Patienten sei es zu einer größeren Arbeitsbelastung, besonders für die Pflegekräfte, gekommen (A9).

Sieben Ärzte sprechen die Mehrarbeit durch das Kodieren an (A2, A4, A5, A7, A8, A9, A10). Es würden pro Tag durchschnittlich 20-30 Minuten mehr Arbeit am Computer anfallen (A9) und es gebe wenige Möglichkeiten, sich für das häufig sehr komplizierte und zeitaufwendige Kodieren fortzubilden (A2). 


\section{DARSTELLUNG DER UNTERSUCHUNGSERGEBNISSE}

Durch die Einführung von Fallpauschalen und dem damit verbundenen Qualitätsmanagment habe die Politik einen Weg gefunden, die Krankenhauslandschaft [durch Klinikschließungen] zu verändern (A8). Ein Arzt endet mit den Worten: „Es wird sich noch herausstellen, ob dieses System positiv zu bewerten ist.“(A6).

\subsubsection{Zusammenfassung}

Seit der DRG-Einführung stellen die befragten Ärzte einen erhöhten Aufwand an Dokumentation fest. Es gibt ihrer Meinung nach eine Tendenz zur ambulanten Behandlung von Lungenkrebspatienten, so dass das Patientenkollektiv, das stationär versorgt wird, kränker erscheint, da die gesünderen Patienten nun ambulant versorgt werden. Die Liegezeit der Patienten hat sich nach Angaben der Ärzte verkürzt, was für den einzelnen Patienten von Vorteil sein kann, da er nicht unnötig lange im Krankenhaus bleiben muss.

Die subjektive durchschnittliche Dauer der Aufklärungsgespräche liegt zwischen 10 und 60 Minuten. In den drei an der Studie teilnehmenden Häusern gibt es unterschiedliche Durchschnittswerte (Universitätsklinikum Göttingen: 45 Minuten, Krankenhaus Lenglern 30 Minuten, Evangelisches Krankenhaus Weende 20 Minuten). Der errechnete Durchschnittswert der Dauer des Aufklärungsgesprächs beträgt für die drei Kliniken 36 Minuten.

In dem Aufklärungsgespräch werden die Patienten aus ärztlicher Sicht über alle Behandlungsoptionen aufgeklärt, oft ist es aber für den behandelnden Arzt nicht einfach zu eruieren, ob der Patient auch alle Informationen aus diesem Gespräch verstanden hat. Im Idealfall sollte der Patient nach dem Gespräch selbst beurteilen können, warum eine bestimmte Therapieform ausgewählt wird.

In der Diagnostik, Therapie und Prognose von Patienten mit einem Bronchialkarzinom haben sich aus Sicht der befragten Ärzte seit dem Jahr 2004 keine wesentlichen Veränderungen ergeben. Die Therapieoptionen der einzelnen Patienten werden in einer Tumorkonferenz, an der alle behandelnden Institutionen aus dem Raum Südniedersachsen teilnehmen, im Rahmen der Richtlinien für Tumorpatienten festgelegt. Dabei spielt es keine Rolle, ob der Patient gesetzlich versichert oder ein Selbstzahler ist. Von ärztlicher Seite wird versucht, auf die individuellen Wünsche und Bedürfnisse einzugehen, dies gelingt aber nicht immer. Entlassungen für ein 


\section{DARSTELLUNG DER UNTERSUCHUNGSERGEBNISSE}

Wochenende $\mathrm{zu}$ ermöglichen, ist schwieriger geworden. Ansonsten bestehen aber keine Unterschiede zu der Zeit vor der DRG-Einführung.

Die Einführung der DRG bewerten die Ärzte überwiegend positiv. Sie sehen in dem neuen Abrechnungssystem ein Modell, das hilft, Kosten einzusparen und das transparenter als das bisherige ist. Zur Zeit gibt es aus ärztlicher Sicht keine Verschlechterung in der Versorgung von Bronchialkarzinompatienten.

Gleichzeitig sehen die Ärzte aber auch Schwierigkeiten, die durch das neue Abrechnungssystem entstehen können. Als negativ wird der erhöhte Dokumentationsaufwand bewertet. Außerdem warnen einige Ärzte vor einer schlechten Abbildung bestimmter Patientengruppen wie chronischkranker oder sterbender Patienten.

\subsection{Auswertung der Befragung von Pflegekräften}

Im Rahmen der vorliegenden Studie wurden, analog zu den Interviews mit Ärzten, auch persönliche Interviews mit Pflegekräften durchgeführt. An dieser Befragung nahmen 13 Pflegekräfte (neun weiblich, vier männlich) teil. Die Befragung fand im April 2006 statt und wurde von der Autorin persönlich in teilstrukturierten Interviews durchgeführt (s. Kap. 2.4.)

Einschlusskriterien für die teilnehmenden Pflegekräfte waren: Beschäftigung an einem der drei teilnehmenden Häuser, Erfahrungen im Umgang mit Bronchialkarzinompatienten und Ausübung der pflegerischen Tätigkeit seit mindestens Januar 2003.

\subsubsection{Auswertung der soziodemographischen Daten der Pflegekräfte}

Neun Krankenschwestern und vier Krankenpfleger nahmen an der Befragung teil. Von ihnen sind fünf Personen als Stationsleitung bzw. stellvertretende Stationsleitung tätig. Das Alter der Befragten liegt zwischen 36 und 54 Jahren. Die Pflegekräfte arbeiten zum Zeitpunkt der Befragung zwischen 2 und 30 Jahren (durchschnittlich 11 Jahre) in der jeweiligen Abteilung. Im Evangelischen Krankenhaus Weende wurden ein Jahr vor Durchführung der Interviews (2005) die Stationen neu strukturiert und zum Teil zusammengelegt. Deshalb arbeiten die Pflegekräfte dort erst seit dem Jahr 2005 auf der jeweiligen Station. Sie sind aber schon vor diesem Zeitpunkt in der Pflege von Patienten mit Bronchialkarzinom tätig gewesen. Die befragten Pflegekräfte aus 


\section{DARSTELLUNG DER UNTERSUCHUNGSERGEBNISSE}

dem Universitätsklinikum Göttingen arbeiten auf den Stationen der Hämatologie und Onkologie, im Krankenhaus Lenglern sind sie auf pulmonologischen Stationen und im Evangelischen Krankenhaus Weende auf einer chirurgischen Station tätig.

\subsubsection{Angaben zu den Arbeitsbedingungen}

\subsubsection{Veränderungen der Arbeitsbedingungen und Zufriedenheit}

Im Universitätsklinikum Göttingen lässt sich nach Angaben einer Pflegekraft eine Tendenz zu einem höheren „Patientenumsatz“ (Interview P2) erkennen. Außerdem gibt eine Krankenschwester an, dass die Patienten „häufig kränker sind“ (P3), wenn sie auf Station kommen, da „Patienten, die gesünder sind, mehr ambulant behandelt werden“ (P3). Dieses Phänomen hänge mit der DRG-Einführung zusammen, da seit 2004 vermehrt darauf geachtet werde, möglichst viele Patienten ambulant $\mathrm{zu}$ behandeln (z.B. bei intravenöser Chemotherapiegabe). Der Arbeitsaufwand des Pflegepersonals sei gestiegen, dies könne aber auch mit einem Personalabbau seit dem Jahr 2005 zusammenhängen. Es lässt sich nicht feststellen, ob der höhere Arbeitsaufwand direkt mit der Einführung der DRG zusammenhängt oder ob er durch personelle Veränderungen zustande gekommen ist.

Im Krankenhaus Lenglern wird ebenfalls von einem höheren „Patientenumsatz“ (P6) und einer „höheren Arbeitsdichte“ (P9) berichtet: „Innerhalb kurzer Zeit werden mehr Patienten durchgeschleust“ (P8). Außerdem kämen die Patienten in einem „kränkeren Zustand“ (P10) auf die Stationen. Nach Eindruck der Pflegekräfte im Krankenhaus Lenglern hat sich diese Entwicklung im Jahr 2005 noch verschärft, denn auch in dieser Klinik sind Planstellen nicht besetzt worden, so dass es durch personelle Veränderungen zu einem Mehraufwand an Arbeit gekommen ist.

Im Evangelischen Krankenhaus Weende wurden, auf Grund eines Neubaus, die Stationen 2005 neu gestaltet. Die Stationen sind zum Teil zusammengelegt worden, so dass sich die Bettenkapazität auf den neu entstandenen Stationen um jeweils 10 Betten auf 30 erhöht hat. Durch diese Veränderung kommt es in dieser Klinik zu einem höheren Arbeitsaufwand, denn die Pflegenden sind nun im Durchschnitt für mehr Patienten zuständig. Die Pflegenden des 


\section{DARSTELLUNG DER UNTERSUCHUNGSERGEBNISSE}

Evangelischen Krankenhauses Weende berichten ebenfalls, dass es einen höheren „Umsatz“an Patienten gebe und Patienten nach Operationen schneller entlassen würden.

Pflegekräfte aller Häuser (P3, P5, P6, P12) erwähnen, dass es durch vermehrte Dokumentation zu einer höheren Arbeitsbelastung komme. Der erhöhte Arbeitsaufwand lasse sich aber nicht direkt auf die Einführung der DRG zurückführen, denn es müssen auch strukturelle und personelle Veränderungen berücksichtigt werden.

Die Zufriedenheit mit den vorherrschenden Arbeitsbedingungen fällt individuell unterschiedlich aus. Zwei Pflegekräfte sind zufrieden oder sehen keine Unterschiede zu den Bedingungen vor DRG-Einführung (P1, P9). Drei Pflegende sind seit der DRG-Einführung unzufriedener, da der Arbeitsaufwand gestiegen sei und die Dokumentation zugenommen habe (P5, P7, P10). Ein Krankenpfleger, der seit 15 Jahren in seiner Abteilung im Krankenhaus Weende arbeitet, äußert: „Da kann man nicht zufrieden sein“ (P13). Er bemängelt, dass viele Überstunden durch die Dokumentation entstehen.

\subsubsection{Das DRG-System als Diskussionsthema der Pflegekräfte}

Aus den geführten Interviews wird ersichtlich, dass die Einführung von Fallpauschalen kaum ein Gesprächsthema für die Pflegekräfte der teilnehmenden Häuser ist. Neun Pflegekräfte geben an, darüber wenig bzw. kaum zu sprechen. „Das System wurde ohne größere Diskussion übernommen“(P12). Allenfalls zu Beginn der Einführung wurde darüber gesprochen. Da es auch Pflegediagnosen gibt, die relevant für die Abrechnung sind, werde bei Unklarheiten mit den Kollegen oder Ärzten gesprochen, gibt eine Krankenschwester an (P4).

Allerdings sei die Verweildauer der Patienten ein Thema, über das die Pflegekräfte untereinander sprechen. Die befragten Pflegekräfte sehen einen Zusammenhang von Veränderungen der Verweildauer mit der DRG-Einführung (P2, P5). Eine Pflegekraft wundert sich, dass „,immer noch Patienten länger liegen, als es eigentlich notwendig ist. Das hat aber abgenommen.“(P5). 


\subsubsection{Veränderungen für die Patienten}

Die Pflegekräfte machen im folgenden Abschnitt Angaben zu Veränderungen, die sich ihrer Meinung nach seit Einführung der DRG für onkologische Patienten ergeben haben. Dazu zählen Angaben über Aufnahme- und Entlassungskriterien, Medikation und Unterschiede in der Behandlung von Kassenpatienten und Selbstzahlern.

Eine Stationsleitung aus dem Universitätsklinikum Göttingen berichtet zum Thema Aufnahmeund Entlassungskriterien: „Man bemüht sich, schneller zu entlassen oder mehr ambulant zu versorgen, onkologische Patienten werden aber so lange wie nötig im Krankenhaus belassen“ (P2). Außerdem seien die stationär behandelten Patienten seit der DRG-Einführung kränker (P3) und die Überwachung sei intensiver (P2). Die meisten Patienten würden aus den Kliniken direkt nach Hause oder in eine Anschlussheilbehandlung entlassen (P12).

Für die befragten Pflegekräfte ist die Art der Versicherung von untergeordneter Relevanz. Es gebe zwar kleine Unterschiede, z.B. würden geplante Eingriffe bei Selbstzahlern schneller durchgeführt, aber die Art der Therapie hänge bei allen Patienten von der jeweiligen Diagnose und nicht von der Art der Krankenversicherung ab (P2). Ein Unterschied sei die Möglichkeit der Wahl eines Einzelzimmers für Selbstzahler und eine Betreuung durch den Chefarzt (P7). Ein Pfleger sagt, dass ,an Selbstzahler von Pflegeseite distanzierter herangegangen“ wird (P13) und eine andere Pflegekraft gibt an, dass sich „Privatpatienten“ vielleicht subjektiv besser behandelt fühlten, dies objektiv aber nicht so sei (P10).

Die Medikation und die verwendeten Präparate ändern sich nach Angaben der Befragten häufig. Das sei aber nicht erst seit DRG-Einführung so. Die Zuständigkeit, welches Präparat in dem jeweiligen Krankenhaus verwendet werde, obliege der Apotheke. Die Pflegekräfte berichten, dass seit Einführung der DRG nicht mehr oder weniger Medikamente verordnet wurden.

\subsubsection{Veränderungen der pflegerischen Arbeit}

Neun der befragten Pflegekräfte berichten, dass die Dokumentationsarbeit sich in den letzten Jahren vergrößert habe. Diese Veränderung hänge aber ihrer Meinung nach nicht ausschließlich mit DRG zusammen, für die sie Pflegediagnosen eintragen müssten, sondern auch mit einer 


\section{DARSTELLUNG DER UNTERSUCHUNGSERGEBNISSE}

umfassenderen Dokumentation aller Pflegeleistungen. So wurde z.B. im Universitätsklinikum Göttingen 2003 ein neues Pflegedokumentationssystem (Leistungserfassung in der Pflege, LEP) eingeführt, in dem seit 2003 alle pflegerischen Leistungen mit Hilfe eines Computerprogramms erfasst werden. Außerdem gebe es strukturelle Veränderungen, da auf einigen Stationen neue Stationsleitungen eingesetzt wurden und es dadurch neue Pflegeanweisungen gebe. So gehören z.B. das Anhängen von Antibiosen oder Blutentnahmen wieder zu den pflegerischen Tätigkeiten.

\subsubsection{Individuelle Patientenbetreuung}

Die Pflegenden machen unterschiedliche Angaben darüber, wie viel Zeit sie sich für Patienten und persönliche Gespräche mit ihnen nehmen können. Durch den erhöhten Dokumentationsaufwand und die höhere Arbeitsdichte ,gibt es tendenziell weniger Zeit, Gespräche mit den Patienten zu führen“(P4). Es bestünde aber nach wie vor die Möglichkeit dazu. Besonders in Krisensituationen „wird sich Zeit genommen“(P3), um mit den Patienten zu reden (P4). Eine Krankenschwester berichtet, dass „Gespräche z.B. stattfinden, wenn Verbände gewechselt werden“, dass aber „mehr Raum für Gespräche wünschenswert ist“ (P5). Ein Pfleger berichtet, dass ,im Pflegegesetz von 1987 nur 57 Sekunden für ein Patientengespräch vorgesehen sind“ (P9). Diese Aussage zeigt, dass es nicht erst seit Einführung der DRG wenig Zeit für Gespräche mit Patienten gibt. Acht Pflegekräfte geben an, dass es zwar nicht immer leicht sei, sich Zeit für Patienten zu nehmen, dies aber trotzdem getan werde.

Im Universitätsklinikum und im Evangelischen Krankenhaus Weende gibt es zur Betreuung onkologischer Patienten nach Angaben der Pflegekräfte außerdem den psychoonkologischen oder seelsorgerlichen Dienst. Im Krankenhaus Lenglern wird dieser Dienst ehrenamtlich geführt. In diesem Krankenhaus versuchen die Stationsleitungen, sich etwas mehr aus dem Pflegealltag herauszunehmen, um mehr Zeit für Gespräche mit den Patienten zu haben (P9).

Die Pflegekräfte bekommen, nach eigenen Angaben, häufig ein positives Feedback von den Patienten. Eine Schwester meint dazu: „Über das Negative redet man nicht“ (P1). Unzufrieden seien die Patienten meistens, wenn ihnen die Wartezeit für diagnostische Maßnahmen zu lang erscheine. Aus dem Krankenhaus Lenglern berichtet eine Stationsleitung, die dort seit dem Jahr 2000 arbeitet, dass die Patienten, die schon länger kommen, es ,früher gemütlicher und ruhiger“ 


\section{DARSTELLUNG DER UNTERSUCHUNGSERGEBNISSE}

(P7) in diesem Krankenhaus empfunden hätten. Durch Gespräche und Erklärungen könnten aber häufig „die Wogen geglättet werden“(P13), um die Unzufriedenheit der Patienten so im Rahmen zu halten.

Eine Krankenschwester sagt, dass die Pflegekräfte sich nach wie vor eine individuelle Betreuung der Patienten „leisten“ (P9), eine andere berichtet: „Es gibt keine Zeit mehr, um z.B. mit Patienten nach draußen zu gehen.“ (P8). Insgesamt versuchen die Pflegekräfte, auf die Wünsche und Bedürfnisse einzugehen, durch den gestiegenen Arbeitsaufwand sei dies aber nicht immer möglich. Die administrativen Vorgaben der Häuser erlauben die Erfüllung bestimmter Wünsche nicht mehr oder erschweren sie. An der Liegezeit z.B. könne man nicht viel ändern, da die Betten immer wieder für neue Patienten benötigt würden (P12). Eine andere Krankenschwester meint, dass sie eigentlich gar nicht auf individuelle Bedürfnisse eingehen dürfe, da eine individuelle Betreuung nicht von den Krankenkassen vergütet werde (P3). Fünf Pflegekräfte (drei aus dem Universitätsklinikum, zwei aus dem Krankenhaus Lenglern) berichten, dass es im Vergleich zu dem Zeitraum vor Einführung der DRG keine Veränderung gebe und sie nach wie vor auf die individuellen Wünsche der Patienten eingehen können (P1, P2, P5, P7, P9).

\subsubsection{Leitlinien}

Für die Pflege von Tumorpatienten gibt es nach Angaben der befragten Pflegekräfte Richtlinien. Dies sind z.B. Leitlinien für die Pflege während einer Chemotherapie oder für spezielle Mundpflege und die Ernährung von Krebspatienten (P1, P2, P6). Die Zeitabläufe auf den Stationen werden nach Angabe der Pflegekräfte aber individuell je nach Pflegebedürftigkeit des Patienten eingeteilt und nicht durch derartige Leitlinien vorgeschrieben (P1, P2). Eine genaue Dokumentation dieser Leistungen sei besonders zur Kontrolle der Richtlinien wichtig (P6).

\subsubsection{Zusammenarbeit mit Ärzten}

Die Zusammenarbeit mit den Ärzten wird in den einzelnen Kliniken unterschiedlich beurteilt. Im Göttinger Universitätsklinikum wird die Zusammenarbeit von vier Pflegekräften als positiv bewertet, wobei zwei von ihnen anmerken, dass die Zusammenarbeit durch die schnelle Rotation 


\section{DARSTELLUNG DER UNTERSUCHUNGSERGEBNISSE}

der Ärzte auf den Stationen schwierig sei (P4, P5). Zum Teil wünschen sich die Pflegekräfte bessere Absprachen über die Auslastung der Stationen. Oft sei es nicht durchführbar, Patienten ein Einzelzimmer zu ermöglichen, da „die Ärzte die Zimmer voll haben wollen“ (P1). Für wichtig wird befunden, gemeinsame Visiten abzuhalten (P2).

Im Krankenhaus Lenglern wird die Zusammenarbeit positiv bewertet. Ein Pfleger berichtete von einem „fruchtbaren Austausch“(P6). Eine Stationsleitung merkt aber an, dass der Pflege oft das Verständnis für das „Durchschleusen der Patienten“ (P7) fehle, da für sie eher die Pflege und nicht der finanzielle Aspekt im Vordergrund steht.

Im Evangelischen Krankenhaus Weende bewerten die Pflegekräfte die Zusammenarbeit weniger positiv. „Könnte manchmal besser sein“ (P12) äußert eine Schwester. Eine andere sagt, dass kaum miteinander kommuniziert werde und von den Ärzten wenig Rücksicht auf die Situation der Pflegekräfte genommen werde (P13).

\subsubsection{Persönliche Beurteilung der DRG-Einführung}

Die Pflegekräfte haben die Möglichkeit, ihre eigene Einschätzung zur DRG-Einführung abzugeben. Sechs Pflegekräften bewerten das neue System als positiv bzw. nicht schlechter als das alte Abrechnungssystem (P1, P3, P4, P7, P10, P12). Eine Schwester äußert: „Eigentlich ist es nicht schlechter als vorher, DRG sind nicht das Schlechteste, wenn das System von allen Seiten gut durchdacht ist.“ (P4) Als positiv wird die durch das neue System eingeführte Transparenz bewertet (P1, P7, P9). Es könne jetzt besser als vor DRG-Einführung abgesehen werden, wie teuer eine Behandlung ist (bzw. sein sollte). Dadurch könnte einer Verschwendung von Gesundheitsgütern entgegengewirkt werden. Eine Änderung des alten Systems sei wichtig gewesen, weil es von vielen Seiten, besonders in Bezug auf die Liegezeiten, ausgenutzt wurde (P5). Wenn durch das DRG-System eine Kostenreduktion gewährleistet werde, sei dieses System sinnvoll, aber dies dürfe nicht zu Lasten der Patienten geschehen (P13).

Als negativ bewertet eine Pflegekraft, dass Patienten häufiger aus kleinen Häusern in größere Zentren überwiesen würden, da die Behandlungskosten für die kleinen Häuser nicht mehr tragbar seien (P2). Eine Krankenschwester sagt, dass ihrer Meinung nach das DRG-System für die 


\section{DARSTELLUNG DER UNTERSUCHUNGSERGEBNISSE}

Pflegekräfte und die Patientenversorgung schlecht sei (P8), da weniger Zeit für eine persönliche Betreuung bleibe.

\subsubsection{Zusammenfassung}

Nach Angaben der befragten Pflegekräfte ist es seit der DRG-Einführung zu einem gestiegenen Arbeitsaufwand und $\mathrm{zu}$ vermehrtem Dokumentationsaufwand gekommen. Die Zeit für eine individuelle Betreuung des Patienten hat abgenommen, trotzdem versuchen die Pflegekräfte auch weiterhin, genügend Zeit für eine individuelle Patientenbetreuung $\mathrm{zu}$ finden. Die Unterschiede zwischen Kassenpatienten und Selbstzahlern (sofern vorher vorhanden) hätten nicht zugenommen.

Über die Zufriedenheit der Pflegekräfte mit ihren derzeitigen Arbeitsbedingungen lässt sich keine pauschale Aussage treffen. Die Angaben gehen von „ich bin zufrieden“ bis „,damit kann man nicht zufrieden sein“. Inwieweit die Einführung der DRG im Zusammenhang mit dem gestiegenen Arbeitsaufwand und der Zufriedenheit mit den bestehenden Arbeitsbedingungen steht, lässt sich aus den geführten Interviews nicht sicher eruieren. Das Pflegepersonal hat die Einführung ohne große Diskussionen akzeptiert und steht Neuerungen offen gegenüber.

Die persönliche Bewertung des DRG-Systems durch die Pflegekräfte fällt unterschiedlich aus. Negativ wird das Fehlen einer persönlichen Betreuung der Patienten bewertet. Als positiv bewerten die Pflegekräfte, dass die Leistungen im Krankenhaus transparenter abgebildet werden und sich die Liegezeiten der Patienten verringert haben. Dabei ist den Pflegekräften aber wichtig, die Gesundheitskosten nicht nur zu Lasten der Patienten zu senken. Von den erwarteten Veränderungen nach DRG-Einführung steht für die Pflegekräfte die Verkürzung der Verweildauer im Vordergrund. Durch diese Änderung werden ihrer Meinung nach mehr Patienten in der gleichen Zeit behandelt, was $\mathrm{zu}$ einem Mehraufwand führt. Andere Veränderungen, z.B. durch vermehrte Dokumentation oder Stellenstreichungen, stehen nicht in direktem Zusammenhang mit der DRG-Einführung, führen aber zu einer höheren Arbeitsbelastung und zum Teil auch zu Unzufriedenheit der Pflegenden. 


\subsection{Auswertung der Controlling-Daten des Universitätsklinikums Göttingen}

Neben den subjektiven Daten der schriftlichen und persönlichen Befragungen werden im folgenden Absatz Daten der Stabsstelle Controlling des Universitätsklinikums Göttingen vorgestellt. Die Stabsstelle Controlling hat für die vorliegende Studie Daten von Patienten zusammengestellt, die in den Jahren 2003-2005 mit der Diagnose „Bronchialkarzinom“ im Universitätsklinikum behandelt wurden. Diese Daten dienen dazu, die subjektiven Aussagen der Patienten, Ärzte und Pflegekräfte mit objektiven Daten zu vergleichen und Gemeinsamkeiten bzw. Unterschiede herauszuarbeiten. Für die vorliegende Studie sind folgende Daten in Bezug auf die ethische Fragestellung relevant:

- Fallzahl der stationären Behandlungen

- Mittlere Verweildauer der Patienten

- Letalität im Krankenhaus.

\subsubsection{Datenanalyse Bronchialkarzinompatienten}

Tabelle 3.4.1a gibt einen Überblick über die Fallzahl, mittlere Verweildauer und Letalitätsrate aller Patienten, die im Universitätsklinikum Göttingen in den Jahren 2003-2005 mit der Diagnose „Bronchialkarzinom“ stationär behandelt wurden.

\begin{tabular}{|l|l|l|l|}
\hline Jahr & Fallzahl & $\begin{array}{l}\text { Mittlere } \\
\text { Verweildauer }\end{array}$ & $\begin{array}{l}\text { Krankenhausletalität } \\
\text { (Prozent der im Klinikum verstorbenen } \\
\text { Patienten mit Bronchialkarzinom) }\end{array}$ \\
\hline $\mathbf{2 0 0 3}$ & 227 Fälle & 12,4 Tage & $10,8 \%$ \\
\hline $\mathbf{2 0 0 4}$ & 224 Fälle & 11,4 Tage & $10,3 \%$ \\
\hline $\mathbf{2 0 0 5}$ & 220 Fälle & 11,8 Tage & $9,1 \%$ \\
\hline
\end{tabular}

Tabelle 3.4.1a Fallzahl, mittlere Verweildauer und Krankenhausletalität der Diagnose „Bronchialkarzinom“ des Universitätsklinikums Göttingen 2003-2005 


\section{DARSTELLUNG DER UNTERSUCHUNGSERGEBNISSE}

In den drei untersuchten Jahren hat die Fallzahl von 227 um 7 Fälle auf 220 Fälle abgenommen. Dies entspricht einer Abnahme von 3\%. Die Studienergebnisse aus den USA hatten eine signifikante Abnahme der Überweisungen in die stationäre Behandlung gezeigt (s. Kap. 1.4.2.). Die mittlere Verweildauer der Patienten nimmt laut der Daten der Stabsstelle Controlling von 12,4 Tagen im Jahr 2003 auf 11,4 Tage im Jahr 2004 ab, steigt aber im Jahr 2005 wieder auf 11,8\%. Die Letalitätsrate von Bronchialkarzinompatienten im Universitätsklinikum Göttingen nimmt von 10,8\% im Jahr 2003 auf 9,1\% im Jahr 2005 ab.

Für die Diagnose „Bronchialkarzinom“ gibt es mehrere DRG, die den Kliniken zur Erlöserrechnung dienen. Diese können in operative und konservative DRG eingeteilt werden. In Tabelle 3.4.1b sind die Fallzahl der einzelnen Jahre in operative und konservative Fälle eingeteilt. Im Folgenden wird anhand eines Beispiels kurz erläutert, wie komplex die DRG-Einteilung für die Diagnose „Bronchialkarzinom“ ist und welche Veränderungen sich in den Jahren 2003 bis 2005 allein für diese Diagnose ergeben haben:

Im Jahr 2003 gab es 16 DRG, welche die Diagnose „Bronchialkarzinom“ kodieren konnten. Davon waren fünf DRG für operative Eingriffe mit unterschiedlichem Schweregrad festgelegt. So gab es z.B. die DRG mit der Nummer E01A: „große Eingriffe am Thorax mit äußerst schwerer CC (CC = Komplikationen oder Komorbiditäten)“. Diese DRG wurde in den Katalog für 2004 übernommen, im Jahr 2005 aber nicht mehr aufgenommen. Zur besseren Abbildung der tatsächlich anfallenden Kosten hat das InEK im Jahr 2005 die DRG mit der Nummer E01Z: „Revisionseingriffe, beidseitige Lobektomie und erweiterte Lungenresektionen“ und DRG E05A: „Andere große Eingriffe am Thorax bei bösartiger Neubildung“ an Stelle der nicht übernommenen DRG E01A eingeführt. Ein Patient, dessen Diagnose 2004 mit der DRG Nummer E01A kodiert wurde und ein Patient, der 2005 den gleichen Eingriff und die gleiche Diagnose hat, bekommt eine andere DRG-Nummer zugewiesen.

\begin{tabular}{|l|l|l|l|l|}
\hline Jahr & Fallzahl ges. & $\begin{array}{l}\text { Operativ } \\
(\text { Fälle })\end{array}$ & $\begin{array}{l}\text { Konservativ } \\
(\text { Fälle) }\end{array}$ & $\begin{array}{l}\text { Nicht klassifizierbar } \\
(\text { Fälle) }\end{array}$ \\
\hline $\mathbf{2 0 0 3}$ & 227 & 68 & 36 & 123 \\
\hline $\mathbf{2 0 0 4}$ & 224 & 68 & 155 & 1 \\
\hline $\mathbf{2 0 0 5}$ & 220 & 71 & 143 & 6 \\
\hline
\end{tabular}

Tabelle 3.4.1b Aufteilung der Fälle in operative und konservative Fälle im Vergleich 2003-2005 


\section{DARSTELLUNG DER UNTERSUCHUNGSERGEBNISSE}

Aus Tabelle 3.4.1b wird ersichtlich, dass 2004 und 2005 ca. ein Drittel (68 von 224 Fällen im Jahr 2004 bzw. 71 von 220 Fällen im Jahr 2005) der Fälle im Universitätsklinikum Göttingen chirurgisch und zwei Drittel (155 Fälle im Jahr 2004 und 143 Fälle im Jahr 2005) konservativ behandelt wurden. Im Jahr 2003 waren 123 Fälle, also mehr als die Hälfte, noch nicht zu klassifizieren. Das hängt damit zusammen, dass in diesem Jahr mit der Kodierung über das DRGSystem begonnen wurde und das System noch nicht für alle Fälle anwendbar war. Dass die Anzahl der operativen Fälle in den drei Jahren konstant geblieben ist, könnte darauf hinweisen, dass die nicht klassifizierbaren Fälle des Jahres 2003 zu den konservativ behandelten Fällen zählen. Da sich diese Vermutung aber nicht verifizieren lässt, ist eine weitere Unterteilung der Daten in operative und nicht-operative DRG nur für den Zeitraum 2004 und 2005 sinnvoll. In diesen beiden Jahren soll die mittlere Verweildauer (VWD) in Tagen verglichen werden:

\begin{tabular}{|l|l|l|l|}
\hline Jahr & $\begin{array}{l}\text { Mittlere VWD } \\
\text { (in Tagen) }\end{array}$ & $\begin{array}{l}\text { Mittlere VWD (operativ) } \\
\text { (in Tagen) }\end{array}$ & $\begin{array}{l}\text { Mittlere VWD (konservativ) } \\
\text { (in Tagen) }\end{array}$ \\
\hline $\mathbf{2 0 0 4}$ & 11,4 & 21,1 & 7,1 \\
\hline $\mathbf{2 0 0 5}$ & 11,6 & 16,2 & 8,6 \\
\hline
\end{tabular}

Tabelle 3.4.1c Vergleich der mittleren Verweildauer für operative und konservative DRG 2004 und 2005

Diese Tabelle zeigt, dass die mittlere Verweildauer in Bezug auf alle Patienten mit Bronchialkarzinom konstant bleibt (11,4 Tage im Jahr 2004, 11,6 Tage im Jahr 2005), es aber zu einem Rückgang der Verweildauer bei operativen Fällen um durchschnittlich fast 5 Tage kommt. Die Studienergebnisse aus den USA haben einen signifikanten Rückgang der Verweildauer gezeigt. Dieser Rückgang ist in der vorliegenden Studie für operativ versorgte Patienten mit Bronchialkarzinom ebenfalls zu verzeichnen. Der Rückgang der Verweildauer für chirurgische Patienten kann sowohl mit der DRG-Einführung als auch mit verbesserten und schonenderen chirurgischen Operationsverfahren in Zusammenhang stehen.

\subsubsection{Weitere Daten der Analyse}

Folgende Daten, die ebenfalls aus der Stabsstelle Controlling der Universitätsklinik stammen, fließen außerdem in die vorliegende Studie ein: 


\section{DARSTELLUNG DER UNTERSUCHUNGSERGEBNISSE}

- Anzahl der „Kurzlieger“: Patienten, deren Verweildauer unter der unteren Grenzverweildauer liegt;

- Anzahl der „Langlieger“: Patienten, deren Verweildauer oberhalb der oberen Grenzverweildauer liegt;

- Veränderung des „Relativgewichts“. Das Relativgewicht gibt an, um wie viel teurer oder billiger die Behandlung eines Patienten gegenüber dem durchschnittlichen Behandlungsfall ist und ist eine vom Institut für das Entgelt im Krankenhaus (InEK) festgelegte Größe;

- Veränderung des „Effektivgewichts“. Das Effektivgewicht berücksichtigt im Gegensatz zum Relativgewicht die Abschläge bei Kurzliegern bzw. die Zuschläge bei Langliegern;

- Veränderung des „Effektiverlöses“. Der Effektiverlös ist der Erlös, der dem Krankenhaus effektiv für die Behandlung eines Patienten gezahlt wird;

- Veränderungen der „Erlösanteile aus Zusatzentgelten“. Zusatzentgelte werden neben den DRG von den Krankenkassen an die Kliniken ausgezahlt (z.B. für sehr teure Medikamente).

Das „Effektivgewicht“ wird mit einem Krankenhaus-einheitlichen Basisfallpreis multipliziert, um so den Fallerlös zu errechnen. Seit dem Ende der Konvergenzphase 2009 ist der Basisfallpreis (Bundes)land-einheitlich. Um aus dem Fallerlös den Effektiverlös zu berechnen, werden seit $2004 \mathrm{zu}$ dem errechneten Fallerlös noch Zusatzentgelte hinzuaddiert (s. Kap. 2.5.). Eine Auswertung der Veränderung dieser Daten ist wichtig, um das Patientenkollektiv der Bronchialkarzinompatienten genau zu untersuchen. Durch einen Datenvergleich ist es möglich, Veränderungen, die sich im Bereich des Controllings und damit der Abrechnung ergeben haben, darzustellen.

\begin{tabular}{|l|l|l|l|l|l|l|}
\hline Jahr & $\begin{array}{l}\text { Kurzlieger } \\
\text { (Fälle) }\end{array}$ & $\begin{array}{l}\text { Langlieger } \\
\text { (Fälle) }\end{array}$ & $\begin{array}{l}\text { Relativ- } \\
\text { gewicht } \\
\text { (Summe) }\end{array}$ & $\begin{array}{l}\text { Effektiv- } \\
\text { gewicht } \\
\text { (Summe) }\end{array}$ & $\begin{array}{l}\text { Effektiverlös/ } \\
\text { Fall }\end{array}$ & $\begin{array}{l}\text { Erlösanteil aus } \\
\text { Zusatzentgelten }\end{array}$ \\
\hline $\mathbf{2 0 0 3}$ & 2 & 15 & 196,2 & 208,0 & $707,15 €$ & 0 \\
\hline $\mathbf{2 0 0 4}$ & 20 & 14 & 400 & 372,3 & $1265,66 €$ & $0,24 \%$ \\
\hline $\mathbf{2 0 0 5}$ & 27 & 14 & 410,3 & 380,4 & $1273,56 €$ & $0,81 \%$ \\
\hline
\end{tabular}

Tabelle 3.4.1d Vergleich der Daten des Controllings (Kurzlieger, Langlieger, Relativgewicht, Effektivgewicht, Effektiverlös und Erlösanteil an Zusatzentgelten) für die Jahre 2003-2005 
Die Relativ- und Effektivgewichte und damit auch die Effektiverlöse haben sich seit dem Jahr 2003 kontinuierlich gesteigert. 2003 lag das Relativgewicht bei 196,2, das Effektivgewicht bei 208 und der Effektiverlös pro Fall bei 707,15 €. Im Jahr 2005 hat sich das Relativgewicht mit einem Wert von 410,3 mehr als verdoppelt, das Effektivgewicht liegt bei 380,4 und der Effektiverlös bei 1273,56 € pro Fall. Von 2003 auf 2004 ist das Relativgewicht von 196,2 auf 400 gestiegen. Dieser starke Anstieg lässt sich dadurch erklären, dass, wie oben bereits beschrieben, 2003 eine freiwillige Einführung stattgefunden hat und Diagnosen kostenneutral durch die DRG kodiert wurden.

Neben dem effektiven Fallerlös hat auch der Anteil an Zusatzentgelten für die Kostenerstattung von Bronchialkarzinompatienten zugenommen. Diese Zusatzentgelte wurden erst 2004 eingeführt. Sie dienen dazu, besonders teure Medikamente aus der DRG-Bewertung herauszunehmen, um die Höhe der DRG nicht zu verfälschen und diese Medikamente trotzdem allen Patienten zur Verfügung stellen zu können. 


\subsection{Ergebniszusammenfassung anhand der Forschungsfragen}

\subsubsection{Gibt es aus Sicht der Studienteilnehmer eine Veränderung in der Behandlungsqualität von Bronchialkarzinompatienten während der DRG-Einführung?}

Diese Frage wird mit Hilfe der Daten der schriftlichen Patientenbefragung und der Interviews mit Ärzten und Pflegekräften beantwortet. Die Qualität der ärztlichen und pflegerischen Behandlung wird von den Patienten mit „gut“ bis „,sehr gut“ bewertet (s. Tab. 3.1.5 und Tab. 3.1.6). Die Behandlungsqualität verändert sich aus Sicht der Patienten während des untersuchten Zeitraums nicht. In allen drei Jahren werden aber auch Kritikpunkte zur Behandlungsweise geäußert. Dabei spielt vor allem die Zeit der betreuenden Personen für die Patienten eine Rolle. Aus Sicht einiger Patienten nehmen sich die Betreuenden nicht genügend Zeit (s. Kap. 3.1.4.). Die Ärzte und Pflegekräfte geben an, aufgrund des erhöhten „Patientenumsatzes“ weniger Zeit für die Betreuung der Patienten zu haben. Trotzdem geben sie an, sich noch Zeit für die individuelle Patientenbetreuung zu nehmen. Die subjektive Wahrnehmung der Ärzte und Pflegekräfte, dass es zu einem schnelleren Wechsel der Patienten kommt, kann mit den objektiven Daten des Controllings verglichen. Diese lassen einen leichten Rückgang der mittleren Verweildauer von 12,4 Tagen 2003 auf 11,8 Tage 2005 aller im Universitätsklinikum Göttingen behandelten Patienten mit Bronchialkarzinom erkennen. Die Patientengruppe wurde zur weiteren Analyse in chirurgisch und konservativ Behandelte unterteilt. Die Auswertung dieser Daten zeigt, dass die Verweildauer der chirurgisch behandelten Patienten im Jahr 2005 um durchschnittlich ca. fünf Tage im Vergleich zu 2004 abgenommen hat. Die Verweildauer der konservativ behandelten Patienten bleibt dagegen weitgehend konstant. Für die chirurgischen Patienten ist somit ein deutlicher Rückgang der Verweildauer zu erkennen (s. Tab. 3.4.1c). Die Daten des Controllings bestätigen für die chirurgischen Stationen den Eindruck der Pflegekräfte und Ärzte, dass die Verweildauer der Patienten abgenommen hat und es dadurch bei konstanter Belegungsrate der Station zu einem erhöhten „Umsatz“ von Patienten kommt, wobei aber berücksichtigt werden muss, dass die Fallzahl sogar leicht abgenommen hat und die befragten Pflegekräfte des Universitätsklinikums Göttingen auf einer internistischen Station tätig waren. Auf diesen Stationen ist weder eine Erhöhung der Fallzahl, noch eine kürzere Verweildauer zu erkennen (s. 


\section{DARSTELLUNG DER UNTERSUCHUNGSERGEBNISSE}

Kap. 3.4.1.), so dass die Daten des Controllings mit den Eindrücken der Ärzte und Pflegekräfte nicht übereinstimmen.

Aus ärztlicher Sicht ist es teilweise schwieriger geworden, auf die Bedürfnisse und Wünsche des einzelnen Patienten einzugehen. Die befragten Ärzte sehen dabei aber keine Verschlechterung der Behandlungsqualität durch die DRG-Einführung. Ein Lungenkrebspatient bekommt nach Angaben der Ärzte auch nach Einführung der Fallpauschalen weiterhin die bestmögliche Therapie. Die Pflegekräfte sehen ebenfalls keine Abnahme der Behandlungsqualität. Sie geben allerdings an, dass mehr Raum für Gespräche wünschenswert wäre und dass die Arbeitsbelastung durch vermehrt kränkere Patienten auf den Stationen zugenommen habe. Einige der befragten Pflegekräfte sind mit ihren Arbeitsbedingungen unzufrieden, weil sie nicht genügend auf den einzelnen Patienten eingehen können (s. Kap. 3.3.2.1.).

Aus diesen Aussagen und Daten geht hervor, dass es während des untersuchten Zeitraums aus Sicht der Ärzte und Pflegekräfte zu einem erhöhten Arbeitsaufwand und weniger Zeit für die Betreuung des einzelnen Patienten gekommen ist, sie sehen aber keine Minderung der Behandlungsqualität durch die Einführung von Fallpauschalen. Die Angaben der Patienten lassen ebenfalls keine Veränderung der Behandlungsqualität erkennen.

Inwiefern die Veränderungen der Verweildauer und der Arbeitsbedingungen für Ärzte und Pflegekräfte in Zusammenhang mit der DRG-Einführung stehen, lässt sich aufgrund der vielfältigen Veränderungen im stationären Bereich (Neuordnung von Stationen, neue Stationsleitung, Änderungen in der Dokumentationsarbeit etc.) nicht mit Sicherheit eruieren.

\subsubsection{Verändert sich die Lebensqualität der Patientenpopulation während der Einführung von Fallpauschalen?}

Zur Beantwortung dieser Frage werden die aus dem Standardfragebogen EORTC QLQ-C30 (s. Kap. 3.1.10.) gewonnenen Daten der Funktions- und Symptomskalen ausgewertet. Anhand dieser Skalen kann die Lebensqualität der Patienten multidimensional untersucht werden und eine Aussage über Veränderungen der Lebensqualität während der Studiendauer gemacht werden. Die Lebensqualität der Patientenpopulation dieser Studie nimmt im untersuchten Zeitraum nicht ab.

Die Patienten geben ihre Lebensqualität zum Zeitpunkt der Befragung und nicht zum Entlassungszeitpunkt an. Diese beiden Zeitpunkte können bei den Patienten bis zu einem Jahr 


\section{DARSTELLUNG DER UNTERSUCHUNGSERGEBNISSE}

auseinander liegen, da die Befragung immer die Patienten, die im vorangegangenen Jahr stationär behandelt wurden, berücksichtigt. Da die Patientenbefragung aber in allen drei Jahren in den Monaten Februar bis Mai durchgeführt wurden, lässt sich die Lebensqualität der Patienten aufgrund der durchschnittlich gleichen Zeitspanne miteinander vergleichen. Wegen der geringen Fallzahl scheint eine weitere Unterteilung der Patienten nach dem Zeitpunkt ihrer Entlassung nicht sinnvoll.

Die Werte der Funktionsskala sind im Jahr 2005 durchschnittlich am höchsten. Das bedeutet, dass die Patienten in diesem Jahr am besten ihre alltäglichen Aufgaben verrichten können. Gleichzeitig sind 2005 die Werte der Symptomskalen am niedrigsten, so dass die Patienten in diesem Jahr auch am wenigsten durch Symptome beeinträchtigt sind. Dies ist ein wichtiges Ergebnis im Hinblick auf das Nichtschädigungsprinzip, denn vor DRG-Einführung wurde befürchtet, dass die Patienten aus Kostengründen zu einem früheren Zeitpunkt und in einem schlechteren Gesundheitszustand und somit mit einer schlechteren Lebensqualität entlassen würden. Die Angaben der befragten Patienten können diese Befürchtung nicht unterstützen.

\subsubsection{Verändert sich der Pflegebedarf der Patienten bei Entlassung?}

Um den Gesundheitszustand bei Entlassung zu untersuchen, wurde das Patientenkollektiv zum benötigten Pflegebedarf bei Entlassung befragt. Dabei wurde davon ausgegangen, dass ein geringer Pflegebedarf bei Entlassung mit einem besseren Gesundheitszustand einher geht als ein hoher Pflegebedarf. Der Pflegebedarf der befragten Patienten nach Entlassung hat in den Jahren 2004 und 2005 im Vergleich zum Jahr 2003 (vor DRG-Einführung) nicht zu-, sondern abgenommen (s. Tab. 3.1.8). In den Studien aus den USA hatte der Pflegebedarf bei Entlassung signifikant zugenommen. Dieser Trend lässt sich in der eigenen Studie nicht bestätigen. Nach Auswertung der eigenen Daten wird außerdem sichtbar, dass der größte Teil der Pflege und Betreuung der untersuchten Patienten von den Angehörigen geleistet wird. Trotz der niedrigen Rücklaufquote kann hier ein Trend abgelesen werden.

Anzumerken ist, dass keiner der befragten Patienten angab, dauerhaft in einer Pflegeinstitution untergebracht worden $\mathrm{zu}$ sein. Das hat vermutlich einen systematischen Bias erzeugt; der Fragebogen wird diese Patienten entweder nicht erreicht haben oder diese Gruppe der Patienten hat nicht geantwortet. 


\subsubsection{Verändert sich die stationäre Letalitätsrate der Bronchial- karzinompatienten während der Einführung des DRG-Systems?}

Eine Befürchtung vor der Einführung des DRG-Systems war, dass das DRG-System Anreize schafft, Patienten früher als medizinisch indiziert aus dem Krankenhaus zu entlassen. Dadurch könnte es insgesamt zu einer erhöhten Letalitätsrate kommen. Die amerikanischen Studien kamen zu dem Ergebnis, dass es eine Abnahme der Letalitätsrate im Krankenhaus und eine Verschiebung des Sterbeorts gab. Für die vorliegende Studie liegen die Letalitätsraten für Patienten mit Bronchialkarzinom im Universitätsklinikum Göttingen vor. Laut der Datenanalyse der Stabsstelle Controlling ist die Letalitätsrate von 10,8\% im Jahr 2003 auf 9,1\% im Jahr 2005 gesunken. Durch die eigene Datenanalyse ist keine Aussage über einen möglichen Wechsel des Sterbeorts möglich.

\subsubsection{Kommt es aus Sicht der Studienteilnehmer zu Einschränkungen in der Therapie- und Behandlungsfreiheit von Bronchialkarzinom- patienten?}

In der Onkologie gab es schon vor der DRG-Einführung Leitlinien und Behandlungspfade, die sich aus den Ergebnissen von Studien herleiten. Im Raum Südniedersachsen werden die Therapiemöglichkeiten für Bronchialkarzinompatienten in einer Tumorkonferenz gemeinsam von den an der Therapie beteiligten Ärzten besprochen. Die von Ärzten und Pflegekräften gemachten Aussagen lassen nicht erkennen, dass die Therapiefreiheit in der Behandlung von Patienten mit Bronchialkarzinom durch die DRG-Einführung eingeschränkt wird. Eine überwiegende Zahl der befragten Patienten in allen drei Jahren gibt an, über die Art der Behandlung und Nebenwirkungen aufgeklärt worden zu sein. Allerdings geben 8 der 109 befragten Patienten an, sich an keine Aufklärung über Nebenwirkungen erinnern zu können, und sechs Patienten sind nach eigenen Angaben nicht über die Behandlungsweise aufgeklärt worden (s. Tab. 3.1.4.1b).

Die Ärzte sagen zu diesem Punkt, dass es für sie nicht immer sicher sei, dass der Patient das Wesentliche verstanden habe (s. Kap. 3.2.3.4.). Die Studienteilnehmer sehen insgesamt keine Einschränkung der Therapiefreiheit durch die Einführung des neuen Abrechnungssystems. 


\subsubsection{Können Fallpauschalen individuell auftretende Probleme erfassen und kann ein Patient seinem psychischen und physischen Gesundheitszustand entsprechend behandelt werden?}

Als ein Problem, das durch die DRG-Einführung entsteht, wird die pauschale Abrechnung der Behandlung von Patienten gesehen. Jedem Patienten wird eine bestimmte Fallpauschale zugeordnet, aus der sich der pauschale Erlös der jeweiligen stationären Behandlung errechnet. Die Pauschale ist unabhängig von der jeweiligen individuellen Behandlung des Patienten. In der ethischen Diskussion vor Einführung der Fallpauschalen wurde deshalb befürchtet, dass es durch eine fallpauschalisierte Abrechnung zur Benachteiligung von schwerkranken und sterbenden Patienten kommen könnte (s. Kap. 1.5. und Kap. 1.5.2.), denn Gespräche oder die Betreuung von sterbenden Patienten werden in dem neuen Abrechnungssystem nicht gesondert vergütet. Dabei stellt sich die Frage, ob bei einer pauschalen Vergütung auch die Behandlung ,pauschal“ abläuft. Dies würde bedeuten, dass Ärzte und Pflegekräfte nicht genügend auf den individuellen Gesundheitszustand eingehen können.

Das DRG-System soll zu einer verbesserten Transparenz der stationären Behandlung im Krankenhaus führen. Wenn aber individuellen Leistungen wie Gespräche und Sterbebegleitung in diesem System gar nicht erst abgebildet werden, kommt es zu einer Benachteiligung derjenigen Patienten, deren Behandlung in besonderem Maße von diesen Leistungen abhängt.

Um die Frage nach Veränderungen der individuellen Betreuung zu beantworten, werden die Daten zu Gesprächen, der Zufriedenheit mit diesen Gesprächen und zur individuellen Patientenbetreuung vor und während der DRG-Einführung herangezogen. Dabei werden sowohl die Daten der Patientenbefragung wie auch die Interviews mit Ärzten und Pflegekräften berücksichtigt.

Die Dauer des Aufklärungsgesprächs wird von den befragten Ärzten mit durchschnittlich 36 Minuten angegeben. Die befragten Patienten geben an, dass die durchschnittliche Dauer des Aufklärungsgesprächs im Jahr 2003 bei 30 Minuten liegt, im Jahr 2004 bei 15 Minuten und im Jahr 2005 bei 20 Minuten (s. Abb. 3.1.4). Aus diesen Angaben lässt sich kein eindeutiger Trend ablesen.

Ein befragter Arzt empfindet, dass er zu wenig Zeit für Gespräche habe (A11). Er gibt eine durchschnittliche Gesprächsdauer von 10 Minuten an. Dagegen sagen fünf der befragten Ärzte, 


\section{DARSTELLUNG DER UNTERSUCHUNGSERGEBNISSE}

dass die Dauer des Aufklärungsgespräches immer vom Patienten abhängt und sie sich auch nach DRG-Einführung soviel Zeit für ein Gespräch nehmen würden, wie es der jeweilige Patient benötigt (A2, A5, A6, A7, A8).

Die Rolle des Arztes als Gesprächspartner ist den befragten Patienten dieser Studie besonders wichtig. In jedem Jahr der Befragung geben mehr als 60\% der Studienteilnehmer an, mit ihrem Klinikarzt über die eigene Sorgen und Ängste sprechen zu können (s. Abb. 3.1.7b).

Durch eine Abnahme der Zeit für den Patienten kann die betreuende Person diese wichtige Aufgabe der psychosozialen Betreuung nicht mehr oder nur in geringem Umfang wahrnehmen. In diesem Zusammenhang sind die Aussagen der befragten Pflegekräfte interessant. Sie geben an, dass sie sich Zeit für Gespräche mit den Patienten nehmen, dies aber durch den höheren Patientenumsatz und die vermehrte Dokumentationsarbeit nicht mehr so leicht sei.

Bei vielen Patienten bestehen Sorgen und Ängste, die von Pflegekräften und Ärzten durch den Mangel an Zeit nicht aufgefangen werden. Deshalb sollte die Beurteilung der Einführung von DRG in der Onkologie und die damit verbundene korrekte Kostendeckung auch den Aspekt der psychosozialen Betreuung mitberücksichtigen. In den an der Studie teilnehmenden Häusern gibt es einen psycho-onkologischen Dienst bzw. ehrenamtliche Mitarbeiter, die sich neben Pflegekräften und Ärzten um die psychosoziale Betreuung der Patienten kümmern. Wie wichtig Gespräche im Klinikalltag sind, verdeutlicht folgende Aussage einer Pflegekraft: „Durch Gespräche und Erklärungen können aber häufig die Wogen geglättet und die Unzufriedenheit der Patienten so im Rahmen gehalten werden“(P13).

Die Ärzte und Pflegekräfte beurteilen die Einführung des DRG-Systems insgesamt als notwendig und gut, da es ihrer Meinung nach richtige und wichtige Anreize in Bezug auf die Verweildauer setzt. Sie äußern aber auch Befürchtungen, dass durch Fallpauschalen weniger auf die individuellen Bedürfnisse und Sorgen eingegangen werden kann. Die befragten Patienten sind auch während der DRG-Einführung mit der individuellen Versorgung in der Klinik zufrieden und bewerten diese als gut (s. Tab. 3.1.5 u. Tab 3.1.6).

Aus Sicht der an der Studie teilnehmenden Patienten gehen die Ärzte und Pflegekräfte auf ihre persönlichen Wünsche und Bedürfnisse ein. Aus den erhobenen Daten lässt sich kein Unterschied vor und während der DRG-Einführung erkennen. Im Rahmen einer individuellen Betreuung ist den Patienten das persönliche Gespräch besonders wichtig. Die befragten Ärzte und Pflegekräfte sehen keine Benachteiligung von onkologischen Patienten sondern glauben, dass das DRG- 


\section{DARSTELLUNG DER UNTERSUCHUNGSERGEBNISSE}

System in Bezug auf die Verweildauer und die knappen Ressourcen gerecht ist. Im Gegensatz zum Abrechnungssystem nach tagesgleichen Pflegesätzen gibt es nun keinen Anreiz mehr, Patienten länger als medizinisch notwendig in der Klinik zu belassen. Auch dies ist für die individuelle Versorgung der Patienten wichtig, denn die gewohnte häusliche Umgebung kann sich positiv auf die psychische Situation des Patienten auswirken.

Aus den vorliegenden Daten kann abgeleitet werden, dass es aus Sicht der Studienteilnehmer während des untersuchten Zeitraums nicht zu einer schlechteren Versorgung von Patienten mit Bronchialkarzinom kommt. Die Patienten werden auch weiterhin gemäß ihrer individuellen Situation behandelt. Inwieweit diese Tatsache aber dem individuellen Engagement der Ärzte und Pflegekräfte zu schulden ist, kann nicht abgeschätzt werden. In der eigenen Untersuchung lässt sich nicht eruieren, ob das DRG-System die individuelle Betreuung von schwerkranken bzw. sterbenden Patienten korrekt abbildet.

\subsubsection{Haben Patienten, die in ländlichen Regionen wohnen, einen fairen Zugang zu ihrer medizinischen Versorgung?}

Diese Frage untersucht, ob sich die medizinische Infrastruktur und damit der Zugang zur Behandlung während der Einführung von Fallpauschalen verändert. Eine gerechte Verteilung von Ressourcen setzt voraus, das auch der Zugang zur medizinischen Versorgung „gerecht“ verteilt ist (s. Kap. 1.5.4.). Durch die prognostizierte Schließung bzw. das Zusammenlegen und die Spezialisierung von Kliniken könnte sich der Anfahrtsweg von Patienten zu ihrem Behandlungsort verlängern, so dass die Wegstrecke für den individuellen gesundheitlichen Zustand nicht mehr zumutbar ist. Dadurch würde es zu einer Benachteiligung dieser Patienten kommen.

Eine Aussage darüber, welcher Anfahrtsweg für eine schwerkranke Patientenpopulation überhaupt zumutbar ist, kann aus den vorliegenden Daten nicht gemacht werden. In dieser Studie wurde statt dessen verglichen, ob es zu einer durchschnittlichen Verlängerung des Anfahrtsweges kommt und ob die befragten Patienten die Länge als zumutbar empfinden.

Die Angaben der an der Studie teilnehmenden Patienten zeigen, dass es bis zum Jahr 2005 zu keiner Verlängerung des durchschnittlichen Anfahrtswegs kommt (s. Abb. 3.1.9). Die erwarteten Veränderungen der medizinischen Infrastruktur werden sich erst längerfristig bemerkbar machen. 
Die Länge der Wegstrecke, die für die eigene gesundheitliche Situation zumutbar scheint, bewerten die Patienten in den Jahren 2003 und 2005 im Durchschnitt kürzer, als ihr eigener Anfahrtsweg tatsächlich ist. Die Angaben zeigen auch, dass sich das untersuchte Patientenkollektiv bei der Angabe nach der angemessenen Wegstrecke häufig an der tatsächlich zurückgelegten Wegstrecke orientiert.

Da der Median der Länge des zurückgelegten Wegs zur Behandlungsstätte in den Jahren 2003 und 2005 den gleichen Wert hat $(35 \mathrm{~km})$ ist es für die an der Studie teilnehmenden Patienten bis 2005 nicht zu einer Verlängerung des Anfahrtsweges gekommen.

Aus den vorliegenden Daten ist somit bislang in Bezug auf die durchschnittlich zurückgelegte Wegstrecke keine Verschlechterung der Situation der im Raum Südniedersachsen wohnenden Patienten mit Bronchialkarzinom zu erkennen. Diese Aussage kann aber nicht auf eine Allgemeinheit bezogen werden. 


\section{DISKUSSION}

In der vorliegenden Studie wurden Veränderungen der stationären Behandlung während der Einführung von Fallpauschalen exemplarisch an Patienten mit Bronchialkarzinom und ihren betreuenden Ärzten und Pflegekräften untersucht. Die forschungsleitenden Fragen leiten sich ab aus der ethischen Diskussion, die in Deutschland zum Thema Fallpauschalen geführt wurde und den Studienergebnissen aus den USA, die dort während der Einführung von DRG erhoben wurden. Die vorliegende Studie untersucht die DRG-Einführung in Deutschland unter dem Aspekt des Nichtschädigungsprinzips und des Prinzips der Gerechtigkeit. Sie ist die erste Studie, in der die Einführung von Fallpauschalen unter ethischen Aspekten erforscht wird. Die ethische Begleitforschung zu diesem Thema ist wichtig, um die Veränderungen des Systems zu erkennen und die Anreize des DRG-Systems unter moralischen Aspekten zu beurteilen. Diese Beurteilung soll helfen, die Umsetzung der DRG-Einführung konstruktiv zu gestalten. Dabei steht die Sorge um das Wohlergehen bzw. die Nichtschädigung des Patienten und der Gedanke einer gerechten Versorgung im Mittelpunkt.

Der erste Abschnitt dieser Diskussion beschäftigt sich mit der geringen Rücklaufquote und der damit verbundenen fehlenden Allgemeingültigkeit der Aussagen in Bezug auf ein größeres Patientenkollektiv.

Desweiteren werden die empirischen Ergebnisse der Patientenbefragung, der persönlichen Interviews mit Ärzten und Pflegekräften und die Daten des Controllings in Hinblick auf ihre Aussagekraft und die ethischen Prinzipien des Nichtschädigens und der Gerechtigkeit diskutiert.

\subsection{Diskussion zum Studiendesign}

Die Daten der vorliegenden Studie basieren unter anderem auf Daten einer retrospektiven Befragung von Bronchialkarzinompatienten. Diese oftmals schwerkranke Patientengruppe wurde gewählt, weil ihre stationäre Versorgung in besonderem Maße einer individueller Betreuung bedarf, die Raum und Zeit für Gespräche und eine psychosoziale Mitbetreuung lässt (s. Kap. 1.5.1.). Gleichzeitig hat die Befragung von schwerkranken und sterbenden Patienten Nachteile, 


\section{DISKUSSION}

denn zum Teil sind die Patienten aufgrund eines sehr schlechten Allgemeinzustands oder ihres Todes nicht mehr in der Lage, retrospektiv Angaben über ihren Klinikaufenthalt zu machen. Es kann in dieser Studie zu einem systemischen Bias gekommen sein, da nur die Patienten antworten konnten, deren Gesundheitszustand eine solche Beantwortung noch zu ließ. Da besonders für die Gruppe der chronisch Kranken und Sterbenden eine Benachteiligung durch die DRG-Einführung befürchtet wurde (Wilmsen-Neumann 2005), erscheint die Untersuchung einer solchen Gruppe trotzdem sinnvoll und wichtig. Deshalb wird in der vorliegenden Studie in Kauf genommen, dass einige Patienten nicht mehr in der Lage waren zu antworten.

Ein Problem, das daraus resultiert, ist die geringe Rücklaufquote. Von insgesamt 403 angeschriebenen Patienten antworteten nur 109. Das entspricht einer Rücklaufquote von $27 \%$. Der Stichprobenumfang der einzelnen Jahre betrug für die Aussagen über das Jahr 200341 Fragebögen, für 200437 Fragebögen und für 200531 Fragebögen (s. Tab. 3.1.1). Nach Rücksprache mit dem Institut für medizinische Statistik der Universität Göttingen reicht dieser Stichprobenumfang nicht aus, um die gewonnenen Ergebnisse auf eine größere Allgemeinheit zu beziehen. Die regenerierten Daten lassen also keinen Rückschluss auf andere Patienten mit Bronchialkarzinom zu. Sie können aber dazu dienen, exemplarisch Veränderungen darzustellen und diese in die Diskussion über das DRG-System und seine Folgen für onkologische Patienten einfließen zu lassen.

Für eine solche Darstellung ist es wichtig, zunächst den Zustand vor DRG-Einführung zu beschreiben. Dies wurde mit der Befragung zum Klinikaufenthalt 2003 angestrebt. Die beiden weiteren Befragungen beziehen sich auf die stationäre Versorgung während der DRGEinführung. Aufgrund vielfältiger Veränderungen in den an der Studie teilnehmenden Kliniken (z.B. Umbaumaßnahmen, Stellenabbau, vermehrte Dokumentation in der Pflege) lässt sich keine direkte Kausalität des Zusammenhangs von Veränderungen der stationären Behandlung alleine durch die DRG-Einführung beweisen. Diese Problematik wird auch in einer anderen Studie zu Veränderungen durch das DRG-System beschrieben (Buhr 2006). Gründe dafür seien, dass es in den vergangenen Jahren eine Reihe von Strukturveränderungen (z.B. Qualitätssicherung, Katalog ambulant durchführbarer Operationen) gegeben habe, von denen die DRG-Einführung nur der vorläufige Endpunkt sei. 


\section{DISKUSSION}

In der eigenen Studie kann deshalb nur der Zustand während der DRG-Einführung aus Sicht der Patienten und betreuenden Personen beschrieben werden, ohne dass sich eindeutig klären lässt, ob dieser Zustand in direktem Zusammenhang mit der DRG-Einführung steht.

Folgende Verbesserungen im Studiendesign können für weitere Studien mit ähnlicher Fragestellung nützlich sein:

- Aushändigen der Fragebögen zur retrospektive Befragung direkt am Ende des jeweiligen Klinikaufenthalts durch den behandelnden Arzt, um mehr Patienten zu erreichen;

- Anreiz für die Beantwortung des Fragebogens (Aufwandsentschädigung, Geschenk).

Durch diese zwei Punkte könnte eine größere Bereitschaft zur Beantwortung des Fragebogens und somit eine höhere Rücklaufquote erreicht werden. Außerdem hätten dadurch eventuell auch Patienten antworten können, die nach Entlassung in einer Pflegeinstitution untergebracht wurden.

- Erweiterung des untersuchten Zeitraums über das Jahr 2005 hinaus, um die Bedingungen der stationären Behandlung nicht nur zu Beginn, sondern auch im Verlauf der DRG-Einführung und nach Beendigung der Konvergenzphase zu untersuchen.

Die obengenannten Verbesserungsvorschläge konnten auf Grund zu geringer finanzieller und zeitlicher Kapazität nicht in die Tat umgesetzt werden, sollten aber bei weiteren Studien zu diesem Thema mitberücksichtigt werden. In der hier vorliegenden Studie wurden statt dessen zusätzlich zur Befragung der Patienten persönliche Interviews mit Ärzten und Pflegekräften geführt und Daten aus der Stabsstelle Controlling des Universitätsklinikums Göttingen regeneriert. Die gewonnenen Daten aus Interviews, Fragebogenerhebung und Controllingdaten dienen dazu, einen möglichst umfassenden Überblick über das untersuchte Patientenkollektiv zu bekommen. Dabei muss berücksichtigt werden, dass sich die Art der Daten unterscheidet und diese sich nicht immer direkt vergleichen lassen. Die Daten der Fragebogenerhebung und der persönlichen Interviews stellen subjektive Meinungen dar, deren Aussage nicht verallgemeinerbar ist (s.o.). Trotzdem sind diese subjektiven Aussagen wichtig zur Beurteilung von Veränderungen, denn sie zeigen die Schwierigkeiten und Probleme auf, die während der 


\section{DISKUSSION}

DRG-Einführung für die untersuchten Patienten und ihre Ärzte und Pflegekräfte entstanden sind. Nur mit Hilfe dieser subjektiven Einschätzungen kann es gelingen, die ethische Dimension der Einführung von Fallpauschalen zu untersuchen. Die Prinzipien des Nichtschädigens und der Gerechtigkeit dürfen sich nicht nur an theoretischen Maßstäben messen lassen, sondern müssen auch auf die betroffenen Patienten und Berufsgruppen anwendbar sein. Aus diesem Grund ist ihre persönliche Beurteilung wichtig für die Einschätzung der ethischen Aspekte.

Die Daten des Controllings werden als Vergleichsmöglichkeit für die subjektiven Ergebnisse genutzt. Sie können in keinem direkten Bezug zu den durch Interviews und Fragebogenerhebung gewonnenen Daten gesehen werden, sondern sollen zeigen, ob die Eindrücke der Befragten mit den objektiven Daten zusammenpassen oder nicht.

\subsection{Nichtschädigungsprinzip}

Im folgenden Abschnitt werden die Studienergebnisse unter dem Aspekt des Nichtschädigens diskutiert. Das „Nichtschädigen“ ist ein Prinzip des ärztlichen Handelns (s. Kap. 1.5.3.). Das Prinzip fordert, dass Ärzte Handlungen unterlassen, die dem Patienten Schaden zuführen. Beauchamp und Childress schließen dabei auch die Risikovermeidung eines Schadens mit ein. Das Nichtschädigungsprinzip tritt häufig in Konflikt mit dem Prinzip des Wohltuns, weshalb die Bedingungen unter denen das Nichtschädigungsprinzip gilt bereits im Abschnitt 1.5.3. erläutert wurden.

Im Rahmen der Einführung des DRG-Abrechnungssystems in Deutschland wurde von einigen Autoren befürchtet, dass es zu einem Konflikt zwischen der Pflicht des ärztlichen Handelns zur Schadens- bzw. Risikovermeidung und dem neuen Abrechnungssystem kommen könnte. Sie befürchteten unter anderem eine Abnahme der Behandlungsqualität durch die DRG-Einführung (Jonitz 2002, Keil 2001, Kölking 2001, Laschet 2001, Wehkamp 2002, Zaddach 2001). Diese Befürchtung ist darin begründet, dass das neue System Anreize schafft, Patienten möglichst zügig und nach einer möglichst kostengünstigen Behandlung nach Hause zu entlassen. Wenn die Patienten keine adäquate Behandlung erhalten oder in einem unzureichenden Gesundheitszustand nach Hause entlassen würden, käme es zu einem Konflikt zwischen dem DRG-System und dem ärztlichen Prinizip dem Patienten keinen Schaden zuzuführen. Aus diesem Grund untersucht die vorliegende Studie die Versorgungsqualität. Diese bezieht sich nicht nur auf die Versorgung 


\section{DISKUSSION}

während der stationären Behandlung, sondern auch auf den Zustand des Patienten zum Zeitpunkt der Entlassung. Um die Behandlungsqualität beurteilen zu können, ist sie in der vorliegenden Studie multidimensional untersucht worden, und zwar im Hinblick auf:

- Behandlungsdauer

- Pflegebedarf zum Zeitpunkt der Entlassung

- Letalitätsrate

- Lebensqualität

- Dauer und Qualität des ärztlichen Gesprächs

- Veränderungen der Arzt-Patient-Beziehung.

\section{Behandlungsdauer}

Die oben genannten Autoren befürchten, dass die Patienten in möglichst kurzer Zeit wieder aus der Klinik entlassen werden und es dadurch zu einer schlechteren Qualität der Behandlung komme. Zur Untersuchung der Behandlungsdauer wurden Daten aus dem Controlling des Göttinger Universitätsklinikums herangezogen. Daneben wird auch die subjektive Meinung der Ärzte und Pflegekräfte zu diesem Aspekt erläutert, um zu klären, ob sich Veränderungen der Verweildauer auch im klinischen Alltag bemerkbar machen und wie diese beurteilt werden.

In der vorliegenden Studie wurde die mittlere Verweildauer der Patienten mit Bronchialkarzinom untersucht und im Weiteren für chirurgisch und konservativ behandelte Patienten getrennt aufgeführt. Die im Göttinger Universitätsklinikum chirurgisch behandelten Patienten haben im Jahr 2005 eine um fast fünf Tage kürzere mittlere Verweildauer als die Patienten im Jahr 2004. Dagegen bleibt die Verweildauer für die konservativ behandelten Patienten relativ konstant und steigt sogar leicht an. Für die konservativ behandelten Patienten kommt es also durchschnittlich zu keinem Rückgang der Verweildauer.

Auch wenn die Daten des Controllings keinen Hinweis auf eine verkürzte Verweildauer der Patienten auf internistischen Stationen geben, nehmen die befragten Pflegekräfte und Ärzte eine subjektive Verkürzung der Verweildauer wahr und stellen sie in Zusammenhang mit der DRGEinführung. Woher diese Wahrnehmung kommt, lässt sich nicht eruieren, sie wird jedoch von fast allen befragten Ärzten und Pflegekräften angegeben. 


\section{DISKUSSION}

Die Interviewten beurteilen eine Verkürzung der Liegezeit für die Patienten und deren Versorgungsqualität eher positiv. Durch das DRG-System wird ihrer Meinung nach verhindert, dass Patienten länger als medizinisch notwendig in der Klinik bleiben. Die Aussage eines Arztes, dass im neuen Vergütungssystem kein Anreiz mehr bestünde, Patienten länger als nötig in der Klinik zu belassen, impliziert, dass es einen solchen Anreiz im vorherigen System (der Abrechnung nach Tagessätzen) gab. Daher scheint eine Verringerung der Liegezeit im Sinne der Patienten ethisch geboten zu sein, denn einen Patienten aus rein ökonomischem Interesse länger als notwendig in der Klinik zu belassen, steht im Widerspruch zu zwei ethischen Prinzipien: Es widerspricht dem Nichtschädigungsprinzip, wenn der Patient eine unnötige bzw. zu lange Behandlung erhält, da ,,jede unnötige Diagnostik und unnötige Therapie primär ein Risiko für den Patienten darstellt“" (Schroeders v. und Köbberling 2002, S. 431). Außerdem besteht ein Konflikt mit dem Gerechtigkeitsprinzip, denn die vorhandenen medizinischen Ressourcen dürfen nicht verschwendet werden. Statt dessen ist ein verantwortungsvoller Umgang mit den knappen Ressourcen sogar ethisch geboten und ökonomisches Handeln eine Voraussetzung für Ethik im Gesundheitswesen (Kölking 2001).

Auf der anderen Seite kann auch eine frühzeitige Entlassung im Widerspruch zum Nichtschädigungsprinzip stehen, z.B. wenn einem Patienten durch eine frühe Entlassung die medizinisch indizierte Behandlung vorenthalten wird. Im schlimmsten Fall könne dies dem Patienten gesundheitlichen Schaden zufügen. Dann sei es die Aufgabe der Ärzte und Pflegekräfte, auf diese Missstände hinzuweisen (Wehkamp 2002).

Es ist sinnvoll, dem Arzt die individuelle Entscheidung zu überlassen, wie lange ein Patient in stationärer Behandlung bleiben muss. Wie oben erläutert kann sowohl eine zu lange als auch eine zu kurze Liegedauer dem Patienten nützen oder schaden. In seiner Entscheidung über die Länge der Verweildauer sollte der Arzt jedoch unabhängig von ökonomischen Zwängen handeln können. Nach Meinung der befragten Ärzte ist dies auch während der DRG-Einführung der Fall.

\section{Pflegebedarf bei Entlassung}

Der benötigte Pflegebedarf zum Zeitpunkt der Entlassung kann indirekt Aufschlüsse über die Behandlungsqualität geben, wenn davon ausgegangen wird, dass ein geringer Pflegebedarf bei Entlassung mit einem besseren Gesundheitszustand einher geht als ein hoher Pflegebedarf und ein guter Gesundheitszustand mit einer guten Behandlungsqualität. 


\section{DISKUSSION}

Die Studienergebnisse aus den USA legen nahe, dass der Pflegebedarf zum Zeitpunkt der Entlassung zunehmen wird, da das DRG-System Anreize schafft, Patienten möglichst frühzeitig nach Hause zu entlassen. Durch die DRG-Einführung ist in den USA ein schlechterer Gesundheitszustand der Patienten zum Zeitpunkt der Entlassung und gleichzeitig ein erhöhter Pflegebedarf zu verzeichnen.

Die eigenen Daten zeigen keinen Anstieg des Pflegebedarfs nach Entlassung (s. Tab. 3.1.8). Kritisch anzumerken ist, dass die Studienteilnehmer in keinem Jahr angaben, dauerhaft in einer Pflegeinstitution untergebracht zu sein. Die Patienten mit einem besonders hohen Pflegebedarf haben vermutlich in allen drei Jahren nicht an der Befragung teilgenommen. Hier gibt es einen systematischen Bias, der bei einer erneuten empirischen Untersuchung durch ein anderes Studiendesign vermieden werden müsste (s. Kap. 4.1.).

Auch wenn es keine Angaben zur Situation der Patienten in Pflegeinstitutionen gibt, zeigen die Antworten der befragten Patienten, dass sie sich zunehmend Sorgen um ihre Angehörigen und deren Belastung durch die Krankheit und die häusliche Versorgung machen (s. Tab. 3.1.7, Abb. 3.1.7a). Dieser Aspekt ist für die zukünftige Beurteilung des DRG-Systems wichtig, denn der häuslichen und ambulanten Pflege wird eine größere Bedeutung zukommen, wenn die Patienten früher aus der Klinik entlassen werden. Wenn die Versorgungsstrukturen im ambulanten Bereich nicht ausgebaut werden, besteht die Gefahr, dass es zu einer Minderversorgung der ambulanten Patienten kommt. Von den Sozialverbänden wurde bereits vor der DRG-Einführung darauf hingewiesen, dass im Fallpauschalensystem die weitere ambulante Versorgung durch das DRGSystem nicht berücksichtigt wird (Beyer-Rehfeld 2001). Deshalb ist es wichtig, die Versorgungsmöglichkeiten nach Entlassung aus der Klinik auszubauen. Wehkamp schreibt: „Von der Qualität der ambulanten Versorgung wird abhängen, ob die Verlegung [nach Hause] dem Wohl des Patienten dient oder ihm Schaden zufügt“ (Wehkamp 2002, S.397). Eine wichtige Aufgabe der Gesundheitspolitik ist deshalb, die ambulanten Versorgungsstrukturen zu verbessern, damit es nicht zu einer Schädigung von Patienten und damit zu einem Konflikt zwischen dem Prinzip des Nichtschädigens und dem DRG-System kommt. 


\section{DISKUSSION}

\section{Letalitätsrate}

Die Studienergebnisse aus den USA haben gezeigt, dass die stationäre Letalitätsrate abnimmt, es jedoch zu einer Verlagerung des Sterbeorts gekommen ist. Würde es im Zusammenhang mit einer Minderversorgung durch das DRG-System zu einer Erhöhung der Letalitätsrate kommen, so wäre dies als Verstoß gegen das Nichtschädigungsprinzip zu werten.

Die vorliegenden Studie untersucht deshalb die Letalitätsrate der Patienten mit einem Bronchialkarzinom, die im Universitätsklinikum Göttingen verstorben sind. Dabei ist eine Reduktion der stationären Mortalitätsrate von Bronchialkarzinompatienten von 10,8\% im Jahr 2003 auf 9,1\% im Jahr 2005 zu verzeichnen. Daraus lässt sich ablesen, dass es auch in der untersuchten Klinik zu einer Abnahme der Mortalitätsrate gekommen ist. Es lassen sich allerdings keine Rückschlüsse auf eine Verlagerung des Sterbeortes ziehen.

Sollte es zu einer Verlagerung des Sterbeorts kommen, so wäre aber, wie bereits unter dem Aspekt des Pflegebedarfs bei Entlassung diskutiert, ein Ausbau der ambulanten Versorgung wichtig. Sterbebegleitung und die Unterstützung von Angehörigen sind ein wichtiger Bestandteil der medizinischen Versorgung. Findet diese Versorgung nicht in der Klinik, sondern im ambulanten Bereich statt, muss der vermehrte Bedarf gedeckt werden können, ansonsten würde sich die Qualität der Behandlung verschlechtern.

\section{Lebensqualität}

Um weitere Rückschlüsse auf die Behandlungsqualität zu ziehen, evaluieren die an der Studie teilnehmenden Patienten ihre Lebensqualität. Für das Jahr 2005 werden dabei die besten Werte der Lebensqualität erzielt (s. Tab. 3.1.10a, Tab. 3.1.10b). Die Patienten fühlen sich in diesem Jahr am wenigsten durch Symptome beeinträchtigt und können viele ihrer „Funktionen“ gut ausführen. Für die untersuchte Patientenpopulation ist keine Verschlechterung der Lebensqualität nachzuweisen. Wenn davon ausgegangen wird, dass eine gute Lebensqualität zu Hause ein Parameter für eine gute Behandlungsqualität ist, folgert daraus, dass die Qualität der Behandlung aus Sicht der untersuchten Patienten nicht abgenommen hat. Im Sinne des Nichtschädigungsprinzips ist dieses Ergebnis positiv zu bewerten. 


\section{DISKUSSION}

\section{Dauer und Qualität des ärztlichen Gesprächs}

Ein wichtiger Teil der Behandlungsqualität betrifft das persönliche Gespräch. Das bestätigen die Aussagen der befragten Patienten. Dabei ist nicht nur das Aufklärungsgespräch von besonderer Bedeutung, auch Gespräche mit dem Arzt über die eigenen Sorgen und Ängste sind den Studienteilnehmern wichtig. So geben in jedem Jahr über $60 \%$ der Patienten an, dass sie mit ihrem Klinikarzt über Sorgen und Ängste sprechen können (Abb. 3.1.7b).

Zum Aufklärungsgespräch befragt, geben die Patienten in allen drei Jahren gute Noten für die Verständlichkeit dieses Gesprächs. Allerdings zeigen die Anmerkungen der Patienten, dass sie Vorschläge zur Verbesserung eines solchen Gesprächs haben: Der Arzt sollte sich z.B. mehr Zeit für das Gespräch nehmen und weitere Gespräche auch im Beisein von Angehörigen führen. Diese Angaben machen deutlich, dass nicht alle Patienten mit der Durchführung ihres Aufklärungsgesprächs zufrieden sind. Außerdem spielt der Faktor Zeit eine wichtige Rolle. Deshalb werden im Folgenden die Angaben der Patienten zur Dauer der Aufklärungsgespräche von 2003 bis 2005 und die Angaben der Ärzte darüber miteinander verglichen.

Die durchschnittliche Dauer des Aufklärungsgesprächs geht nach Angaben der Patienten von 30 Minuten im Jahr 2003 auf 20 Minuten im Jahr 2005 zurück. Die Ärzte geben im Durchschnitt eine längere Dauer an (36 Minuten). Die Zeitangaben dürfen nicht als objektive Größen gewertet werden, da die Dauer des Gesprächs nicht mit einer Uhr gemessen wurde und die Angaben der Erinnerung der Patienten und Ärzte entspringen. Die gewonnenen Daten zeigen aber, dass es Unterschiede in der zeitlichen Wahrnehmung eines solchen Gesprächs gibt. Wichtig ist dabei nicht die Zeitdauer allein, sondern das Gefühl des Patienten, der Arzt habe sich genügend Zeit genommen. Das bestätigen die Angaben der Ärzte. Diese sind der Meinung, sich so viel Zeit zu nehmen, wie der jeweilige Patient für ein solches Gespräch benötigt. Nur ein Arzt berichtet, zu wenig Zeit für ein ausführliches Gespräch zu haben. Ob dieser Zeitmangel direkte Folge des DRG-Systems ist, lässt sich nicht beweisen.

Aus ärztlicher Sicht werden auch Folgegespräche mit den Patienten geführt. Es zeigt sich also eine unterschiedliche Wahrnehmung zur Frage nach der Quantität und Qualität des ärztlichen Gesprächs. Dazu passen die Angaben der Ärzte, seit DRG-Einführung immer mehr Zeit zum Dokumentieren und dadurch weniger Zeit für die Patienten zu haben. Der Palliativmediziner Wilmsen-Neumann schreibt zur Abbildung von persönlicher Zuwendung durch Fallpauschalen: „Es muss berücksichtigt werden, dass die individuellen Leistungen des Arztes, die persönliche 


\section{DISKUSSION}

Zuwendung, das Gespräch mit dem Patienten und die zum Teil sehr aufwendigen Angehörigengespräche nur unzureichend in die Bewertung des Fallgewichts eingehen“ (Wilmsen-Neumann 2005, S. 25). Durch diese unzureichende Bewertung werden persönlichen Zuwendungen im DRG-System nicht vergütet. Aus ethischer Sicht scheint geboten, dass die individuelle Versorgung des Patienten im DRG-System besser abgebildet und damit auch bezahlt wird, da es sonst nicht zu einer umfassenden Behandlung eines Patienten kommt, bei der der Patient als Mensch und nicht nur als Krankheit gesehen wird. Auch wenn die vorliegende Untersuchung keinen Rückgang der Qualität des ärztlichen Gesprächs erkennen lässt unterstreichen die Ergebnisse die Wichtigkeit des Gesprächs. Aus diesem Grund ist es wichtig, eigene DRG für sterbende und onkologische Patienten zu entwickeln, in denen der tatsächliche Aufwand an persönlicher Zuwendung und Zeit für Gespräche mit berücksichtigt wird.

Ein bemerkenswertes Ergebnis der Patientenbefragung ist, dass in allen Jahren jeweils zwei bis vier Patienten angaben, sich nicht zu erinnern, über die Behandlungsweise bzw. Nebenwirkungen der Behandlung aufgeklärt worden zu sein (Tab. 3.1.4.1b). Sollte dies der Wirklichkeit entsprechen, widerspricht eine unterlassene Aufklärung dem Nichtschädigungsprinzip. Allerdings sind die befragten Ärzte der Meinung, dass es für sie häufig schwierig ist, zu erkennen, ob ein Patient das Wesentliche des Aufklärungsgesprächs verstanden hat. Die Angaben der Patienten müssen also nicht den tatsächlich geführten Gesprächen entsprechen.

\section{Veränderungen in der Arzt-Patient-Beziehung}

Durch das DRG-System können sich neben dem persönlichen Gespräch auch weitere Veränderungen in der Arzt-Patient-Beziehung ergeben. In seinem Artikel „Die ökonomische Logik wird zum Maß der Dinge“ beschreibt Flintrop die Ergebnisse zweier Studien zu Veränderungen durch das DRG-System. Die in diesen Studien befragten Ärzte befürchten eine „Ökonomisierung des Gesundheitswesens“ und dadurch eine Verschlechterung der individuellen Arzt-Patient-Beziehung, aus der viele Kliniker ihre Arbeitsmotivation gezogen haben (Flintrop 2006).

Auffallend bei den Befragten der eigenen Studie ist die Wortwahl, mit der die Patientenbehandlung beschrieben wird. Die Ärzte und Pflegekräfte sprechen in den Interviews davon, dass der „Durchlauf“ an Patienten zugenommen habe und geben an, dass es durch die DRG-Einführung zu einem erhöhten „Umsatz“ an Patienten gekommen ist. Diese Beispiele 


\section{DISKUSSION}

zeigen, dass sich das ökonomische Denken auch in der Sprache wiederfindet. Dabei besteht die Gefahr, dass der Mensch nicht mehr als ein krankes und hilfsbedürftiges Individuum gesehen wird, sondern zu einer Sache oder Krankheit ohne menschliche Bedürfnisse gemacht wird. Hier zeigt sich eine Ökonomisierung des ärztlichen und pflegerischen Denkens. Legt man der ArztPatient-Beziehung ein Menschenbild zugrunde, das den gesamten Menschen mit seinen Wünschen und Bedürfnissen sieht, ist es aus ethischer Sicht wichtig, diesem Trend der Ökonomisierung des Patienten mit einer dem gesamten Menschen zugewandten Behandlung entgegenzuwirken. Deshalb muss im DRG-System das Gespräch zwischen Arzt und Patient in der Vergütung berücksichtigt werden. Nur so kann in Zeiten knapper Ressourcen eine gute ArztPatient-Beziehung entstehen, die dem Wohl des Patienten förderlich ist.

Um den Ärzten mehr Zeit für den Umgang mit ihren Patienten zu geben, erscheint es sinnvoll, einen Teil der Dokumentationsarbeit auf andere Mitarbeiter zu verteilen. Aus diesem Grund entstand in Australien während der DRG-Einführung der Beruf des Kodierers. Die Schaffung einer solchen Berufsgruppe kann die deutschen Ärzte entlasten und ihnen Zeit für den persönlichen Kontakt mit Patienten ermöglichen. Dies würde auch die Patientenzufriedenheit und Compliance fördern, die für eine erfolgreiche Therapie unerlässlich sind.

Ein anderer Aspekt der Ökonomisierung der Patientenbehandlung ist die Optimierung von Behandlungsprozessen durch medizinische Leitlinien und Behandlungspfade. Dadurch sollen überflüssige Diagnostik und Therapien vermieden werden (Schroeders v. und Köbberling 2002). Krüger und Rapp kommen in ihrem Artikel „Ethik im Gesundheitswesen: Behandlungsqualität oberste Priorität“" zu dem Ergebnis, dass es durch die Optimierung von Leistungsprozessen und einer Standardisierung von patientenorientierten Behandlungsabläufen zu Kosteneinsparung bei gleichzeitig qualitativ hochwertiger Versorgung kommen kann (Krüger C und Rapp 2006). Laut Neumann ist ein Vorteil solcher Leitlinien, dass die Abläufe nach vorgegebenen Algorithmen stringenter werden (Neumann 2006). Gleichzeitig befürchtet er genau wie Wehkamp, dass Leitlinien die ärztliche Therapiefreiheit einschränken werden (Neumann 2006, Wehkamp 2002). Durch eine Einschränkung der Therapiefreiheit könnte es zu einer Veränderung in der ArztPatient-Beziehung kommen, denn der Arzt ist dann bei der Wahl der Therapie nicht nur dem Wohl seines Patienten verpflichtet, sondern auch der Leitlinie, die für die Krankheit des Patienten vorgesehen ist. 


\section{DISKUSSION}

In der vorliegenden Studie werden die Ärzte und Pflegekräfte zu einer Leitlinien-basierten Medizin und der damit verbundenen Standardisierung von Behandlungsabläufen befragt. Sowohl Ärzte als auch Pflegekräfte geben an, dass es solche Leit- und Richtlinien zur Behandlung eines Bronchialkarzinoms gibt. Sie richten sich nach der Diagnose und sind zum Beispiel als „Empfehlungen zur standardisierten Diagnostik, Therapie und Nachsorge“ (Tumorzentrum Ludwig Heilmeyer 2006) festgehalten oder können im Internet auf der Homepage der Deutschen Krebsgesellschaft als Leitlinien oder „Studienprotokolle“ nachgelesen werden.

Die befragten Ärzte der eigenen Studie sehen einen Nutzen in den Leitlinien, da sie die Versorgung des Patienten nach dem jeweiligen Stand der Wissenschaft sichern. Eine kritische Auseinandersetzung zu diesem Thema wird von den befragten Ärzten nicht angegeben. Es scheint, dass sich die Ärzte in ihrem Handeln trotz des Einsatzes von Leitlinien und der DRGEinführung nicht eingeschränkt fühlen. Die meisten Ärzte geben in den Interviews an, auch weiterhin auf individuelle Wünsche und Bedürfnisse der Patienten eingehen zu können. Die Befürchtungen, dass eine Standardisierung der Therapie die Autonomie des ärztlichen Handelns in Frage stellt, kann in dieser Studie nicht bestätigt werden. Durch eine Leitlinien-orientierte Medizin kommt es aus Sicht der befragten Ärzte nicht zu einer Schädigung des Patienten, sondern zu einer standardisierten und dadurch verbesserten Behandlung.

Die vorliegende Studie zeigt, dass sich die DRG-Einführung für das befragte Patientenkollektiv mit dem Nichtschädigungsprinzip vereinbaren lässt. Die Aussagen der Patienten, Ärzte und Pflegekräfte sowie die Daten des Controllings zeigen Veränderungen, die sich während der Einführung des neuen Abrechnungssystem ergeben haben. Inwieweit diese Daten direkt mit der Einführung des DRG-Systems zusammenhängen lässt sich nicht abschließend klären. Es bleibt aber festzuhalten, dass sich die Behandlungsqualität, die multidimensional untersucht wurde, nicht verschlechtert hat.

Trotzdem ist es wichtig, die weitere Entwicklung der Abrechnung nach DRG unter ethischen Aspekten zu beobachten, um gegebenenfalls auf ein Nichteinhalten des Nichtschädigungsprinzips hinzuweisen und konstruktive Kritik zu üben. Wichtig ist dabei, dass die Ökonomie nicht als letztendlich rechtfertigende Entscheidungsgrundlage angesehen wird. Es muss zu einer Güterabwägung zwischen effizienter Ökonomie und individueller Patientenbehandlung kommen. 


\section{DISKUSSION}

Der behandelnde Arzt trägt die Mitverantwortung für die zur Verfügung stehenden Mittel und muss lernen, mit ihnen in einer verantwortlichen Art und Weise umzugehen.

\subsection{Gerechtigkeit}

Neben dem Nichtschädigungsprinzip ist die Gerechtigkeit ${ }^{12}$ ein weiteres Prinzip zur ethischen Beurteilung der Einführung von Fallpauschalen. Beauchamp und Childress gehen davon aus, dass eine „gerechte“ Verteilung fair, vergleichbar und angemessen für die jeweilige Situation einer Person sein soll (s. Kap. 1.5.4.). Dabei muss die Begrenztheit der Ressourcen berücksichtigt werden, da die vorhandenen Güter nicht ausreichen, um alle denkbar möglichen medizinischen Behandlungen zu finanzieren. Mit Hilfe der vorliegenden Studie wird deshalb untersucht, ob die Einführung von Fallpauschalen eine vergleichbare und bedarfsgerechte medizinische Versorgung gewährleistet.

Vor und während der DRG-Einführung wurde in Deutschland bereits eine ethische Diskussion zum Thema Gerechtigkeit im Fallpauschalensystem geführt (s. Kap. 1.5.4.). Es werden zwei Arten von Verteilung (Allokation) unterschieden: die Mikro- und die Makroallokation (Marckmann 2004). Die Allokation auf der Mikroebene betrifft die Mittelzuteilungen an einen konkreten Patienten. Hierbei geht es nicht nur um die gerechte Verteilung von materiellen Gütern, sondern z.B. auch um andere Ressourcen wie Zeit und Fürsorge, die dem einzelnen Patienten von ärztlicher und pflegerischer Seite entgegengebracht werden. Auf der Makroebene findet die Aufteilung der vorhandenen Ressourcen auf die einzelnen Teilbereiche der medizinischen Versorgung (stationäre und ambulante Versorgung, Rehabilitation, medizinische Forschung etc.) statt. In den Bereich der Makroallokation fällt auch die Anzahl der in Deutschland bestehenden Kliniken bzw. der vorgehaltenen Betten.

\section{Mikroallokation}

Auf der Mikroallokationsebene werden Probleme bei der Behandlung von schwerkranken und multimorbiden Patienten gesehen (DVfR 2001, Laschet 2001). Neumann schreibt: „Alles das, was man unter dem Begriff „Zuwendung“ zusammenfassen kann, also die zeitaufwendige Pflege

\footnotetext{
${ }^{12}$ Definition von „Gerechtigkeit“, s. Kap. 1.5.4.
} 


\section{DISKUSSION}

von geriatrischen oder chronischen, multimorbiden Patienten, bei denen keine große apparative Leistung mehr gebracht werden können, ist eindeutig unterrepräsentiert“ (Neumann 2006, S. 353). Die persönliche Betreuung dieser Patienten sei im DRG-System nicht genügend abgebildet, urteilt Wilmsen-Neumann. Er ist der Meinung, „dass die individuellen Leistungen des Arztes, die persönliche Zuwendung, das Gespräch mit dem Patienten, die zum Teil sehr aufwendigen Angehörigengespräche nur unzureichend in die Bewertung des Fallgewichts eingehen“ (Wilmsen-Neumann 2005, S.25).

Weil ein pauschaler Betrag für eine bestimmte Diagnose gezahlt wird, könnten die Kosten, die bei der individuellen Versorgung multimorbider oder schwerkranker Patienten anfallen, in einem Fallpauschalensystem nicht gut abgebildet werden. Eine schlechte Abbildung könnte zur Folge haben, dass die pauschale Vergütung die tatsächlich anfallenden Behandlungskosten nicht deckt (Beneker 2006). Dieses Problem wird auch in einem Artikel zur DRG-Einführung in den USA beschrieben, in dem Veatch argumentiert, dass eine gerechte Abrechnung im Rahmen des DRGSystems nur dann stattfinden kann, wenn durch die jeweilige Fallpauschale eine adäquate Abbildung der Kosten geleistet wird (Veatch 1986).

Durch das DRG-System bestehen Anreize, die Behandlung von chronisch Kranken und multimorbiden Patienten aus ökonomischen Gründen abzulehnen. In diesem Fall käme es ebenfalls zu einer Benachteiligung bzw. einer ungerechten Behandlung dieser Patienten (Neumann 2006). Neumann argumentiert, dass für Patienten, bei denen wenig apparativer Aufwand, dafür aber ein großer Pflege- und Zuwendungsbedarf besteht, die pauschale Honorierung nicht ausreiche. Besonders gravierend scheint dies für die palliative Versorgung zu sein, so dass es für Krankenhäuser nicht profitabel ist, Palliativstationen einzurichten oder weiter zu betreiben. Dadurch würde es zu einer schlechteren stationären Versorgung von Sterbenden und somit zu einer Benachteiligung dieser Patientengruppe kommen (Wilmsen-Neumann 2005).

Um die Güte der individuellen Betreuung und damit die gerechte Allokation von Gesundheitsgütern auf der Mikroebene zu untersuchen, wird in der vorliegenden Studie die Frage gestellt, ob das DRG-System die individuell auftretenden Probleme darstellen kann und ob die Patienten ihrer Erkrankung gemäß behandelt werden.

Das Abrechnungssystem ist nur dann „gerecht“, wenn der einzelne Patient nicht durch eine pauschale Bezahlung auch pauschal, sondern seinem Gesundheitszustand entsprechend behandelt werden kann. Trotz pauschaler Vergütung einer Behandlung muss auch im DRG-System die 


\section{DISKUSSION}

Möglichkeit einer individuellen Patientenbehandlung gegeben sein, damit das System als „gerecht" bezeichnet werden kann.

Unter diesem Aspekt wurden unter anderem die Daten der Stabsstelle Controlling des Göttinger Universitätsklinikums untersucht. Aus ihnen wird ersichtlich, dass die Anzahl und die Höhe an Zusatzentgelten seit Beginn der Konvergenzphase gestiegen sind. Zusatzentgelte wurden eingeführt, damit besonders teure therapeutische Möglichkeiten, wie z.B. Chemotherapien, die Höhe einer Fallpauschalen nicht verfälschen und auch weiterhin die Nutzung dieser teuren Therapieverfahren für alle Patienten erhalten bleiben kann (Roeder et al. 2004). An den Daten der Stabsstelle Controlling lässt sich außerdem ablesen, dass die Relativgewichte der Fallpauschalen gestiegen sind. Mit Hilfe der Relativgewichte wird der Erlös einer einzelnen Fallpauschale errechnet (s. Kap. 1.2.1.) Eine Steigerung des Relativgewichts bedeutet, dass für die Klinik ein höherer Erlös errechnet wird. Dies war während der Konvergenzphase wichtig, um zu gewährleisten, dass die einzelnen DRG sich langsam an die tatsächlich anfallenden Kosten für eine stationäre Behandlung angleichen konnten. Damit versucht das InEK, eine Behandlung genauer und damit auch gerechter abzubilden. Trotz der Angleichung an die tatsächlich anfallenden Kosten und der besseren Abbildung von onkologischen Diagnosen im DRG-System zeigen Evaluationsprojekte in der Onkologie, dass nach wie vor ein Mangel bei der adäquaten Abbildung und damit auch bei der Vergütung besteht und weitere Nachbesserungen nötig sind (Krause et al. 2005). Auch im Jahr 2008 war die Abbildung der tatsächlich anfallenden Kosten noch nicht vollzogen. So kommt eine Untersuchung zur DRG-Einführung in der Onkologie im Jahr 2008 zu dem Ergebnis, dass die Differenzierung von Komorbiditäten im DRG-System nicht genügend abgebildet wird (Franz et al. 2008). Für die eigenen Daten lässt sich eine vermehrte Differenzierung der Abbildung der Behandlung von Bronchialkarzinompatienten erkennen. Andere Studien machen aber deutlich, dass es weiterhin zu Nachbesserungen in der Abbildung kommen muss, damit die Patienten ihrem Krankheitsbild entsprechend gerecht behandelt werden.

Die Angaben der für die vorliegende Studie befragten Patienten zeigen keine Verschlechterung, sondern sogar eine leichte Verbesserung der Behandlungsqualität. Die oben genannten Veränderungen haben also aus Sicht der Patienten bislang nicht zu einer Verschlechterung in der stationären Behandlung geführt. Die befragten Patienten geben an, dass die behandelnden Ärzte auch während der DRG-Einführung auf individuelle Wünsche und Bedürfnisse eingehen können. 


\section{DISKUSSION}

Die Noten dieser Bewertung und der Bewertung der ärztlichen Betreuung erreichen 2005 sogar die besten Werte (s. Kap. 3.1.5.).

Dagegen empfinden die befragten Pflegekräfte und Ärzte, dass es zum Teil schwieriger geworden ist, die Patienten individuell zu betreuen. Sie begründen dies mit einem erhöhten Arbeitsaufwand. Diese Aussage wird durch Studien zur Veränderung der Patientenversorgung und des Klinikalltags durch die Einführung von DRG bestätigt. Diese Studien sind während der DRGEinführung durch die Universität Bayreuth sowie das Wissenschaftszentrum Berlin in Zusammenarbeit mit der Universität Bremen durchgeführt worden. Die befragten Ärzte und Pflegekräfte berichten auch in diesen Studien, dass nach DRG-Einführung weniger Zeit für Patienten und ihre individuellen Bedürfnisse bleibt (Flintrop 2006). Neumann schreibt, dass das DRG-System die technisch-apparative Behandlung besser darstellt, als die „Zuwendung“ zum Patienten (Neumann 2006). Daraus kann resultieren, dass besonders schwerkranke und sterbende Patienten keine adäquate Behandlung erhalten (Wilmsen-Neumann 2005). Wilmsen-Neumann kommt zu dem Schluss, dass es zur Erhaltung einer guten individuellen Patientenbetreuung wichtig ist, „die Sprache ärztlichen und pflegerischen Handelns wiederzuentdecken“ (ebd., S. 27). Die ethische Relevanz liegt seiner Meinung nach nicht nur darin, dass es „ungerecht“ ist, schwerkranken Patienten nicht die nötige Betreuung und Fürsorge entgegenzubringen, sondern auch darin, wie unsere Gesellschaft mit Sterbenden umgeht. Wenn in einem Vergütungssystem für Krankenhäuser die Betreuung von sterbenden Menschen nicht adäquat vergütet wird, lässt dies auf den Umgang der Gesellschaft mit den Schwächsten und Bedürftigsten schließen. Aus diesem Grund fordert Wilmsen-Neumann eine ethische Debatte über Wertepräferenzen der Gesellschaft.

Die in der vorliegenden Studie befragten Ärzte sehen ebenfalls eine schlechte Abbildung von palliativ behandelten Patienten im DRG-System. Keiner von ihnen gibt aber an, dass einem onkologischen Patienten eine Behandlung vorenthalten wird, weil diese zu teuer sei. Nach Ansicht der Pflegenden und Ärzte hat sich die medikamentöse und technische Behandlung der Bronchialkarzinompatienten nicht geändert. In der eigenen Studie kann nach den Aussagen dieser Personengruppe von einer stabilen medikamentösen und apparativen Behandlungsqualität ausgegangen werden. Somit lässt sich aus Sicht der befragten Ärzte und Pflegekräfte keine ungerechte Behandlung erkennen. 


\section{DISKUSSION}

Der Gesundheitsökonom Roeder geht allerdings davon aus, dass bestimmte teure Leistungen in der Behandlung von onkologischen Patienten im DRG-System nicht gut abgebildet werden und diese deshalb nicht bedarfsgerecht angewandt werden können. Er fordert deshalb in seinem „Gutachten im Auftrag der Deutschen Krankenhausgesellschaft 2006“, dass „für Bereiche, die sich auch nach abschließender Prüfung nicht über DRG finanzieren lassen, andere Finanzierungsformen gefunden werden müssen“ (Roeder 2006, S. 18) und schlägt vor, dass die Grundfinanzierung weiter über DRG erfolgen soll und ergänzende zusätzliche Zentrumszuschläge gezahlt werden sollen. Dadurch könnten weiterhin teure und aufwendige onkologische Therapien erhalten bleiben. Auch für die Palliativmedizin werden solche Zuschläge gefordert (Krüger A 2002).

Von Palliativmedizinern sowie Pflegekräften, die auf Palliativstationen tätig sind, wird das Thema Fallpauschalen kontrovers diskutiert. So sagt Eugen Brysch, Geschäftsführer der Deutschen Hospiz Stiftung: „Mit den Fallpauschalen wird der Patient zu einem Fall, der kostengünstig abzuarbeiten ist". Er fordert deshalb Tagespauschalen für Sterbende (Krüger A 2002, S. 1). Wilmsen-Neumann schreibt, dass die Palliativmedizin einen Anstoß für eine „Philosophie der Medizin“ geben solle, um „einen klaren Blick darauf zu erhalten, was das Wesen der Medizin ist und worin die eigentlichen Leistungen des Arztes beruhen“ (WilmsenNeumann 2005, S. 24). Dabei geht er davon aus, dass durch die vielen Subspezialisierungen oftmals die menschliche Person als Ganzes übersehen wird und dass deshalb auch Behandlungen, die Kommunikation, menschliche Zuneigung und Zuspruch beinhalten (wie die Palliativmedizin) in dem DRG-System nicht oder nur unzureichend abgebildet werden. Für das befragte Patientenkollektiv lässt sich keine Benachteiligung während der Studiendauer verzeichnen. Dabei muss kritisch angemerkt werden, dass der Fragebogen Patienten, die auf einer Palliativstation gelegen haben, nicht erreicht hat, so dass in dieser Studie keine Aussagen über die gerechte Behandlung von Patienten auf Palliativstationen gemacht werden können.

Auch in den USA wurde die DRG-Einführung unter dem Gerechtigkeitsaspekt diskutiert. Fleck (1987) geht in seinem Aufsatz „DRGs: Justice and invisible rationing of health care resources” der Frage nach, ob DRG als „gerecht“ bezeichnet werden können. Er argumentiert, dass das DRG-System von Gesundheitsproblemen ausgehe, die durch chirurgische und andere medizinische Behandlungen geheilt werden könnten. Dabei würde aber nicht der gesamte 


\section{DISKUSSION}

Mensch, sondern nur die Krankheit betrachtet. Leistungen wie Beratung, psychologische Hilfestellungen oder Seelsorge würden nicht berücksichtigt.

Die Ergebnisse der vorliegenden Studie zeigen, dass den befragten Patienten das persönliche Gespräch wichtig ist. Sie bedürfen nicht nur einer rein technisch-apparativen Behandlung, sondern auch einer psycho-sozialen Weiterbetreuung, damit sie als „ganzer Mensch“ gesehen werden. Nur eine Behandlung, die die Gesamtsituation der Patienten mitberücksichtigt, kann als eine gerechte Behandlung die fair, vergleichbar und angemessen ist, bezeichnet werden.

\section{Makroallokation}

Nicht nur auf der Mikro-, sondern auch auf der Makroallokationsebene werden Veränderungen durch das DRG-System erwartet. Laut einer Studie der Wirtschaftsberatungsgesellschaft McKinsey droht bis zu 30\% der deutschen Kliniken der finanzielle Ruin (Hehner et al. 2002). Damit würde es zur Veränderung der gesamten deutschen Krankenhauslandschaft kommen. Dies wäre ein Paradigmenwechsel weg von der flächenhaften Versorgung hin $\mathrm{zu}$ spezialisierten Zentren (Lenk et al. 2005). Besonders für schwerkranke Patienten, die dann weitere Anfahrtswege und eine Behandlung ohne das Beisein ihrer Angehörigen in Kauf nehmen müssten, kann dies zu einer ungerechten Behandlung führen. Auf der anderen Seite kann durch die Spezialisierung von Kliniken auch eine Verbesserung der Behandlungsqualität erreicht werden, denn durch die Vorgabe von Mindestmengen darf ein Arzt nur noch solche Behandlungen durchführen, bei denen er die gesetzlich festgelegten Mindestmengenvorgaben erfüllt. Durch die erhöhte Routine des Arztes kann die Behandlungsqualität verbessert werden. In der vorliegenden Studie wird untersucht, ob sich die Länge des Anfahrtswegs für die befragten Patienten während der DRG-Einführung verändert und welchen Anfahrtsweg sie für angemessen halten. Die Ergebnisse zeigen, dass die Patienten durchschnittlich einen Anfahrtsweg von 35 bis 40 Kilometern zurücklegen müssen (s. Abb. 3.1.9). Für schwerkranke Patienten, die oft mehrmals in der Woche ambulante Chemotherapien und anderen Behandlungen erhalten, sind dies lange Wegstrecken. Die befragten Patienten geben jedoch häufig an, dass die für angemessen befundene Wegstrecke mit der tatsächlich zurückgelegten übereinstimmt. Eine Deutung dieser Angaben ist, dass die Patienten es gewohnt sind, diese Strecke zurückzulegen, und sie deshalb keine kürzere Strecken als notwendig ansehen. Es ergibt sich keine signifikante Verlängerung des 


\section{DISKUSSION}

zurückgelegten Weges im Erhebungszeitraum. Während der Studiendauer ist also keine Verlängerung zu verzeichnen. Dies kann mehrere Ursachen haben:

- Der Befragungszeitraum ist kurz, mögliche Veränderungen können erst zu einem späteren Zeitpunkt auftreten.

- Durch die geringe Anzahl der Patienten, die in jedem Jahr befragt wurde, kann es zu Verzerrungen gekommen sein.

- Außerdem wurde die Studie an drei Krankenhäusern durchgeführt, deren Einzugsgebiet von onkologischen Patienten schon vor Einführung der Fallpauschalen einen großen Radius umfasste (Göttinger Umland, Harz, Solling, Eichsfeld, Nordhessen).

Ob es durch eine potentielle Verlängerung des Anfahrtsweges zu einer ungerechten Behandlung von Patienten kommt, lässt sich aus den vorliegenden Daten nicht schließen. Deshalb ist es wichtig, die DRG-Einführung auch unter der Frage der gerechten Verteilung weiter zu untersuchen und in größer angelegten Studien die längerfristigen Auswirkungen z.B. von Krankenhausschließungen darzustellen.

\section{Legitimation des DRG-Systems durch eine öffentliche Diskussion}

Die in den USA zur DRG-Einführung verfassten Artikel zum Thema Gerechtigkeit kommen zu dem Schluss, dass eine gerechte Verteilung von knappen Ressourcen immer auch durch die Öffentlichkeit legitimiert werden muss. Daneben beschäftigen sich die Autoren mit dem Thema der Rationalisierung, also der Steigerung der Effizienz der erbrachten Leistungen:

- Flecks Kritik (1987) am DRG-System bezieht sich vor allem auf die Tatsache, dass DRG keine offene Diskussion über Rationalisierungsmaßnahmen zulassen, sondern die Rationalisierung „unsichtbar“ vonstatten gehe. In diesem Fall gebe es dafür keine öffentliche Legitimation, und das System wäre als ungerecht zu bewerten.

- Agich (1987) argumentiert in seinem Artikel „Incentives and Obligations under Prospective Payment“, dass der Anspruch der Ärzte an den Ressourcen, die zur Erfüllung des Patientenwohls notwendig sind, immer wieder moralisch gerechtfertigt sein müsse, da diese 


\section{DISKUSSION}

Güter der Gesellschaft und nicht dem Arzt persönlich „gehörten“. Die Verpflichtung des Arztes gegenüber dem Patienten rechtfertige deshalb moralisch keine Verschwendung von Ressourcen. Um den Gebrauch zu rechtfertigen und somit gerecht zu gestalten, solle die Gesellschaft ihr Einverständnis zum Gebrauch der von ihr finanzierten Ressourcen geben.

In Deutschland fehlte bislang die in den amerikanischen Aufsätzen geforderte gesellschaftliche Diskussion und somit die moralische Legitimation des DRG-Systems. Selbst die an der Studie teilnehmenden Pflegekräfte geben an, kaum Gespräche über das neue System geführt zu haben.

Die ärztliche Diskussion zum Thema DRG beschäftigt sich vor allem mit dem erhöhten Dokumentationsaufwand und der damit verbundenen Mehrarbeit. Die Einführung dieses Systems wird aber trotz der vermehrten Dokumentation von den befragten Ärzten eher begrüßt. Die Ärzte sehen in der Abrechnung nach Fallpauschalen die Möglichkeit, Kosten zu senken und eine größere Transparenz der eigenen Arbeit zu erlangen. Unter ethischen Aspekten, wie z.B. der Gerechtigkeitsfrage, wurde die ärztliche Diskussion allerdings nicht geführt.

Die Entscheidung, wie eine gerechte Verteilung aussehen kann, sollte nicht nur von Ärzten, Ökonomen und Statistikern gefällt werden, sondern muss auf der mehrheitlichen Meinung der Bevölkerung, was als „gerecht“ zu bezeichnen ist, basieren. Nur so kann es zu einer moralischen Legitimation dieses Systems kommen. Dabei sollte aber nicht nur das DRG-System Thema einer öffentlichen Diskussion sein, sondern viel allgemeiner der Umgang mit knappen Ressourcen und den gesellschaftlichen Anforderungen an ein gerechtes Gesundheitssystem. Auch die politische Debatte sollte ehrlich und offen geführt werden, damit der Bevölkerung die Problematik und die Folgen von knappen Ressourcen vor Augen geführt werden.

Wenn die flächendeckende Gesundheitsversorgung durch Hausärzte und Kliniken nicht mehr gewährleistet werden kann, ist es wichtig, alternative Modelle zu entwickeln und dabei die Bedürfnisse der Bevölkerung und besonders der schwerkranken und sterbenden Patienten mit einzubeziehen, um weiterhin ein gerechtes und solidarisches Gesundheitssystem aufrechterhalten zu können.

Die Einführung des DRG-Systems erfolgte mit dem Ziel, das deutsche Gesundheitssystem effizienter zu gestalten, um die Kosten so gering wie möglich zu halten. Sie kann als 


\section{DISKUSSION}

Rationalisierungsprozess verstanden werden. Die Gesetzgeber versuchen, die begrenzt vorhandenen Ressourcen „gerecht“ auf das deutsche Gesundheitssystem zu verteilen. In anderen europäischen Ländern, wie z.B. Schweden, wurde zur Rationalisierung von Gesundheitsleistungen eine Prioritätensetzung durchgeführt (Swedish Health Care and Medical Priorities Commission 1993). Dem Vorhaben, Prioritäten zu setzen, um eine möglichst gerechte medizinische Versorgung der gesamten Gesellschaft zu ermöglichen, liegt die Einsicht zugrunde, dass „nur wenige medizinische Leistungen [...] unersetzbar, nicht alle in gleichem Maße zweckmäßig, einige [...] von geringem Nutzen, manche überflüssig oder sogar schädlich“ sind, dass einige Gesundheitsprobleme zum Teil oder im Ganzen in die Eigenverantwortlichkeit der Patienten gehören, andere eine Kostenbeteiligung nahelegen, viele aber einer gesicherten solidarischen Finanzierung bedürfen (Zentrale Ethikkommission 2000, S. 1018).

Um eine gerechte medizinische Versorgung weiterhin zu ermöglichen, ist es auf Grund knapper Ressourcen wichtig, Prioritäten für die Rationalisierung von Gesundheitsleistungen zu setzen, die Eigenverantwortlichkeit der Bevölkerung zu stärken und den Entscheidungsprozess durch einen öffentlichen Diskurs zu legitimieren.

\subsection{Ausblick}

Auch in Deutschland wird mittlerweile über eine Priorisierung im Gesundheitswesen nachgedacht. So hat der Präsident der Deutschen Ärztekammer Hoppe im Mai 2009 eine ehrliche und offene Debatte zu diesem Thema gefordert. Er hält es für unvermeidbar, über den Ausschluss bestimmter Leistungen aus dem Therapiekatalog der gesetzlichen Krankenversicherungen zu diskutieren. Als Beispiel führt er das oben beschriebene Schwedische Modell der Prioritätensetzung an (Rabbata und Meißner 2009).

Auch das Deutsche Ärzteblatt beschäftigt sich seit Anfang 2009 mit der „Priorisierung im Gesundheitswesen“ und veröffentlicht regelmäßig Artikel zu diesem Thema.

Es scheint also auch in Deutschland eine Diskussion über die Legitimation von Rationalisierungsmaßnahmen und Priorisierung zu beginnen. Dies ist ein wichtiger Schritt, um die gerechte Verteilung von knappen Ressourcen zu fördern. Diese Diskussion sollte weiterhin offen und in Zusammenarbeit mit Medizinern, Ethikern, Wirtschaftsexperten und der Politik geführt werden. 


\section{ZUSAMMENFASSUNG}

In der vorliegenden Studie wurden die Auswirkungen der DRG-Einführung im deutschen Krankenhauswesen exemplarisch für Patienten mit Bronchialkarzinom im Raum Südniedersachsen untersucht. Dabei standen die ethischen Prinzipien des Nichtschädigens und der Gerechtigkeit im Mittelpunkt. Die Daten wurden anhand von Patientenbefragungen, Interviews mit Ärzten und Pflegepersonal sowie einer Datenanalyse der Stabsstelle Controlling des Universitätsklinikums Göttingen generiert.

Die Patientenbefragung fand begleitend zur Einführung von Fallpauschalen in den Jahren 2004 bis 2006 statt. Insgesamt nahmen 109 Patienten, die im Universitätsklinikum Göttingen, dem Evangelischen Krankenhaus Weende und dem Krankenhaus Lenglern stationär mit der Diagnose „Bronchialkarzinom“ behandelt wurden, an der Studie teil. Die Patientenbefragung wurde mit Hilfe eines standardisierten Fragebogens retrospektiv durchgeführt. Ziel war es, Veränderungen durch das neue Abrechnungssystem in der Patientenversorgung zu untersuchen.

Zusätzlich wurden Experteninterviews mit Ärzten und Pflegekräften im Zeitraum von November 2005 bis April 2006 durchgeführt. Sie sollten in diesen Interviews zu ihren Arbeitsbedingungen und den Veränderungen seit der Einführung der fallpauschalisierten Abrechnung im Jahr 2004 Stellung nehmen. Um neben diesen subjektiven Einschätzungen und Bewertungen objektive Daten zu generieren, wurde eine retrospektive Datenanalyse von Patienten mit der Diagnose „Bronchialkarzinom“ für den Zeitraum 2003 bis 2005 in Zusammenarbeit mit der Stabsstelle Controlling des Universitätsklinikums Göttingen durchgeführt. Die empirische Untersuchung sollte ein möglichst umfassendes Bild der stationären Versorgung von Patienten mit Bronchialkarzinom wiedergeben. Dabei wird kein Anspruch auf Allgemeingültigkeit erhoben, sondern eine exemplarische Darstellung des Zustands für die untersuchte Patientengruppe erreicht, an deren Beispiel sich Veränderungen und Probleme während der DRG-Einführung aufzeigen lassen. 


\section{ZUSAMMENFASSUNG}

\section{Ethische Implikationen}

Aus den vor der DRG-Einführung erwarteten bzw. befürchteten Veränderungen wurden ethisch relevante Forschungsfragen entwickelt. Dabei standen die Prinzipien des Nichtschädigens und der Gerechtigkeit im Mittelpunkt.

\section{Nichtschädigungsprinzip}

Die in der vorliegenden Studie gewonnenen Daten geben Auskunft über Veränderungen der stationären Behandlung während der DRG-Einführung. Dabei wurde untersucht, ob die Veränderungen im Konflikt zu dem Nichtschädigungsprinzip stehen. Als Messparameter diente die Qualität der Behandlung. Die Qualität wurde multidimensional untersucht:

- Für den Pflegebedarf bei Entlassung ist kein Anstieg zu verzeichnen. Die vorliegende Studie zeigt, dass die Patienten im untersuchten Zeitraum keinen erhöhten Pflegebedarf hatten. Die Behandlungsqualität der stationären Versorgung hat also in Bezug auf den Zustand bei Entlassung nicht abgenommen.

Die Verweildauer hat für die chirurgisch behandelten Patienten abgenommen. Wenn die Verweildauer der Patienten - nicht nur in chirurgischen Abteilungen - weiter abnimmt, ist es für die zukünftige Beurteilung des DRG-Systems wichtig, die Versorgungsstrukturen in den ambulanten Bereichen weiter auszubauen.

- In der vorliegenden Studie lässt sich ein Rückgang der Letalitätsrate für Bronchialkarzinompatienten im Klinikum Göttingen von 10,8\% im Jahr 2003 auf 9,1\% im Jahr 2005 verzeichnen. Ob es allerdings auch wie in den Studien aus den USA zu einer Verlagerung des Sterbeortes kommt, lässt sich durch die vorliegenden Daten nicht klären.

Sollte dies aber zutreffen, wäre, wie oben bereits erwähnt, ein Ausbau der ambulanten Versorgungsstrukturen wichtig. Die Bewertung der Behandlungsqualität unter dem Aspekt der Sterberate kann in der vorliegenden Studie nicht abschließend vorgenommen werden.

- Dagegen geht aus den vorliegenden Daten hervor, dass die Lebensqualität der befragten Patienten im Jahr 2005 am besten bewertet wird. Die Patienten haben in diesem Jahr der Befragung die wenigsten Symptome und können ihre alltäglichen Aufgaben gut ausführen. Wenn davon ausgegangen wird, dass eine gute Lebensqualität nach Entlassung aus der Klinik 


\section{ZUSAMMENFASSUNG}

ein Parameter für eine gute Behandlungsqualität ist, lässt sich daraus schließen, dass die Qualität der Behandlung nicht abgenommen hat. Im Sinne des Nichtschädigungsprinzips ist dieses Ergebnis positiv zu bewerten.

- Die Qualität des ärztlichen Gesprächs wird in allen drei Jahren der Befragung von den Patienten mit guten Noten bewertet. Die Patienten machen aber auch Vorschläge zur Verbesserung, die besonders die Dauer und die Verständlichkeit des Gesprächs betreffen. Für die Behandlungsqualität ergeben sich in Bezug auf das Gespräch zwischen Arzt und Patient aus Sicht der Patienten keine Veränderungen. Die meisten Ärzte geben an, dass sie sich unabhängig von der DRG-Einführung so viel Zeit für wichtige Gespräche (Diagnosemitteilung) nehmen, wie der jeweilige Patient benötigt.

- In der zwischenmenschlichen Beziehung zwischen Ärzten bzw. Pflegekräften und ihren Patienten werden von den in der Klinik tätigen Berufsgruppen Veränderungen durch die DRG-Einführung angegeben. Sie empfinden einen größeren „Umsatz“ oder „Durchlauf“ von Patienten. Dabei erscheinen zwei Aspekte bemerkenswert. Erstens kommt es ihrer Meinung nach durch das neue Abrechnungssystem zu vermehrter Arbeit, weil mehr Patienten in kürzerer Zeit behandelt werden müssen. Zweitens zeigt sich eine Ökonomisierung in der Wortwahl der Ärzte und Pflegekräfte. Legt man der Arzt-Patient-Beziehung ein Menschenbild zugrunde, das den gesamten Menschen mit seinen Wünschen und Bedürfnissen in den Mittelpunkt stellt, ist es aus ethischer Sicht wichtig, diesem Trend der Ökonomisierung der Behandlungssituation entgegenzuwirken. Eine Möglichkeit wäre, das Gespräch zwischen Arzt bzw. Pflegekraft und Patient in der Vergütung mit zu berücksichtigen. So könnte das DRG-System eine gute und umfassende Behandlungsqualität gewährleisten.

- In der vorliegenden Studie wurden die Ärzte und Pflegekräfte nach einer Leitlinien-basierten Medizin und der damit verbundenen Standardisierung von Behandlungsabläufen befragt. Die befragten Ärzte sehen einen Nutzen in den Leitlinien, da sie die Versorgung des Patienten nach dem jeweiligen Stand der Wissenschaft sichern. Die befragten Ärzte geben dabei nicht an, dass die Leitlinien sie in ihrer täglichen Arbeit einschränken. 


\section{ZUSAMMENFASSUNG}

Die vorliegende Studie zeigt, dass sich die DRG-Einführung für das befragte Patientenkollektiv mit dem Nichtschädigungsprinzip vereinbaren lässt. Die Aussagen der Patienten, Ärzte und Pflegekräfte, sowie die Daten des Controllings lassen Veränderungen durch das neue Abrechnungssystem erkennen. Durch die Veränderungen ergibt sich aber keine Verschlechterung der Behandlungsqualität.

Es scheint trotzdem wichtig, die weitere Entwicklung der Krankenhausversorgung unter ethischen Aspekten zu beobachten, um zu gewährleisten, dass es auch weiterhin nicht zu einer Verletzung des Nichtschädigungsprinzips kommt. Dabei sollten neben Gesundheitsökonomen besonders die an der Behandlung beteiligten Personen einbezogen werden. Wichtig ist, dass weiterhin die Behandlung des einzelnen Patienten und nicht die ökonomischen Faktoren im Mittelpunkt stehen. Ärzte und Pflegekräfte müssen dabei lernen, auf eine verantwortungsvolle Weise mit den ihnen anvertrauten Ressourcen umzugehen.

\section{Gerechtigkeit}

Eine gerechte Verteilung wird daran gemessen, ob sie fair, vergleichbar und angemessen ist (Beauchamp, Childress 2001). Dabei werden zwei Verteilungsebenen (Allokationsebenen) unterschieden: Mikro- und Makroallokation.

Die Veränderungen durch das DRG-System in Bezug auf die gerechte Verteilung auf der Mikroebene wurden unter dem Aspekt der individuellen Patientenversorgung untersucht.

- Mit den Daten der vorliegenden Studie kann indirekt auf die Frage der korrekten Abbildung und damit auf die Möglichkeit des Systems eingegangen werden, individuelle Probleme zu erkennen und sie zu behandeln. Die Daten der Stabsstelle Controlling des Göttinger Universitätsklinikums zeigen, dass die Anzahl und die Höhe an Zusatzentgelten seit Beginn der Konvergenzphase gestiegen sind. Die Daten zeigen außerdem, dass die Relativgewichte der Fallpauschalen gestiegen sind, die DRG sich also langsam an die tatsächlich anfallenden Kosten für eine stationäre Behandlung angeglichen haben. In der Literatur zu diesem Thema wird trotz dieser Angleichungen postuliert, dass für onkologische Diagnosen auch im Jahr 2008 noch keine adäquate Kostenabbildung bestehe (Franz et al. 2008). Die entstehenden Behandlungskosten werden also nicht angemessen vergütet. Somit besteht hier auf der Mikroallokationsebene ein Konflikt mit dem Prinzip der Gerechtigkeit. 


\section{ZUSAMMENFASSUNG}

- Die befragten Patienten verspüren ihrerseits keine Verschlechterung, sondern eine Verbesserung der Behandlungsqualität und sind der Meinung, dass die Ärzte auch während der DRG-Einführung auf ihre individuellen Wünsche und Bedürfnisse eingehen können. Die Patienten geben außerdem an, Sorgen und Ängste zu haben und diese mit ihrem behandelnden Klinikarzt besprechen zu können. Aus Patientensicht kommt es während der DRG-Einführung zu keiner ungerechten individuellen Betreuung. Einen besonderen Stellenwert hat für sie dabei das Gespräch mit dem Klinikarzt.

- Die Pflegekräfte und Ärzte berichten, dass eine individuelle Patientenbetreuung eher schwieriger geworden sei. Als Begründung für diese Tatsache führen sie den seit der DRGEinführung gestiegenen Arbeitsaufwand an. Die befragten Ärzte geben außerdem an, dass keinem Patienten durch die DRG-Einführung eine stationäre Behandlung aus Kostengründen vorenthalten wird.

Die Aussagen der Ärzte und Pflegekräfte lassen nicht auf eine ungerechte Behandlung des individuellen Patienten schließen, auch wenn sie durchaus systembedingte Veränderungen und Erschwernisse wahrnehmen. Aus Sicht der Patienten besteht während des untersuchten Zeitraums sogar eine leichte Verbesserung der individuellen Betreuung. Ein wichtiger Aspekt dieser Betreuung ist das Gespräch zwischen Arzt bzw. Pflegekraft und Patient. Eine angemessene, faire und vergleichbare Behandlung ist nur möglich, wenn Ärzten und Pflegekräften genügend Raum für persönliche Gespräche mit dem Patienten bleibt.

\section{Makroallokation}

Die Veränderungen auf der Makroallokationsebene wurden in Hinblick auf die flächendeckende Versorgung untersucht. Die Patienten wurden zu ihrem tatsächlich zurückgelegten Anfahrtsweg zur behandelnden Institution befragt und sollten gleichzeitig angeben, welche Wegstrecke sie für angemessen hielten.

- Nach Angaben der Patienten ergibt sich eine durchschnittlich zurückgelegte Strecke von 3540 km. Für diese meist schwerkranken Patienten scheint dies ein langer Anfahrtsweg zu sein. Trotzdem geben die Patienten häufig an, dass sie die tatsächlich zurückgelegte 


\section{ZUSAMMENFASSUNG}

Anfahrtsstrecke auch als zumutbar empfinden. Sie sehen also keine ungerechte Behandlung. Die Beurteilung, ob diese Strecke als angemessen gesehen werden kann, ist durch diese Studie nicht abschließend zu klären, da die Angaben der Patienten weder angeben, dass sich die Wegstrecke verlängert hat, noch wurden die Patienten direkt gefragt, ob sie sich durch die Länge der Wegstrecke benachteiligt fühlten.

- Ein Vergleich der zurückgelegten Wegstrecken zeigt keine signifikante Verlängerung der Wegstrecke während der DRG-Einführung. Während der Studiendauer ist also keine Verlängerung des Anfahrtswegs zum Behandlungsort zu verzeichnen.

Bei der Bewertung der gerechten Behandlung auf der Makroallokationsebene lässt sich keine Veränderung im Vergleich zur Zeit vor der DRG-Einführung feststellen. Allerdings ist auch hier eine weitere Beobachtung und ethische Diskussion wichtig, denn die durch das DRG-System bedingten strukturellen Veränderungen werden sich zum Teil erst in den kommenden Jahren zeigen. 


\section{ANHANG}

\section{Das Fallpauschalengesetz und seine Änderungen}

\section{Gesundheitsreform}

- die Bundesregierung beschließt die Einführung von Fallpauschalen

2001 Gründung des Instituts zur Selbstverwaltung (InEK gGmbH)

- u.a. zur Weiterentwicklung des DRG-Kataloges zuständig

- für die Durchführung der Begleitforschung zuständig (lernendes System)

2002 Fallpauschalengesetz (FPG)

- das Gesetz zur Einführung von Fallpauschalen wird verabschiedet

- Konvergenzphase bis 2007 beschlossen

2003 Budgetneutrale Einführung

- die Kliniken können auf freiwilliger Basis das Abrechnungssystem anwenden (,Optionsmodell“)

- Fallpauschalenänderungsgesetz (1. FPÄndG)

\section{2. Fallpauschalenänderungsgesetz (2. FPÄndG)}

- Verlängerung der Konvergenzphase bis 2009

- Einführung einer Obergrenze für Konvergenzverlierer

- verbindliche budgetneutrale Einführung der Fallpauschalen in allen Kliniken

- Zusatzentgelte für hochteure Medikamente und Blutprodukte

- Zusätzliche Vergütung von „,neuen Untersuchungs- und Behandlungsmethoden“ 
2005 Beginn der Konvergenzphase

- Schrittweise Einführung der Festlegung landeseinheitlicher Basisfallwerte (evtl. später bundeseinheitlich)

- Sachgerechte Vergütung von Kurz- und Langliegern

- Komplikationen werden organspezifisch kodiert

- Case-Mix-Veränderungen durch Aufsplitten von einfachen und komplexen Fällen

- Versuch, die Zahl der notwendigen DRG zu begrenzen und klinisch heterogene Gruppen zusammenzufassen

\section{2. Jahr der Konvergenzphase}

- Vereinbarung zur Bestimmung von besonderen Einrichtungen (VBE); diese Einrichtungen sind von der Vergütung durch Fallpauschalen ausgenommen (z.B. Palliativhäuser)

- über 954 verschiedene Fallgruppen sind entstanden

- bessere Abbildung der Komplexität von Chemotherapien

\section{3. Jahr der Konvergenzphase}

\section{Ende der Konvergenzphase (1. Januar)}

- es gelten bundeslandweite Basisfallpreise zur Berechnung des Klinikbudgets 


\section{LITERATURVERZEICHNIS}

Aaronson NK, Cull A, Kaasa S, Sprangers MAG: The European Organization for Research and Treatment of Cancer (EORTC) Modular Approach to Quality of Life Assessment in Oncology: An Update; in: Quality of Life and Pharmaoeconomics in Clinical Trials, second Edition; Lippincott-Raven Publishers, Philadelphia 1996, 179-188

Afflerbach F (2002): DRGs - Damoklesschwert oder Silberstreif. Was ist nach aktuellem Stand vom neuen Fallpauschalensystem zu erwarten? . Dtsch Med Wochenschr 127: 187-188

Agich GJ (1987): Incentives and Obligation under Prospective payment. J Med Philos 12: 123144

Beauchamp TL, Childress JF: Principles of biomedical ethics. 5. Auflage. Oxford University Press, Oxford 2001

Beneker C (2006): Krankt die Palliativversorgung am Fallpauschalensystem http://www.aerztezeitung.de/docs/2006/08/03/143a0301.asp Abrufdatum: 09.07.2007

Beyer-Rehfeld A (2001): DRGs verstärken negative Entwicklungen. Sozialdienste fordern stärkere Berücksichtigung sozialer Aspekte. Krankenhaus Umschau 12: 1123

Buccheri G, Ferrigno D (2003): Lung cancer in North-West Italy: demographic and clinical trends in a hospital-based population of 1277 patients. Eur J Cancer Prev $\underline{6}$ : 455-461

Buchner F, Wasem J (2000): Versteilerung der alters- und geschlechtsspezifischen Ausgabenprofile von Krankenversicherern. Wirtschaftswissenschaftliche Diskussionspapiere Nr. 1/00 http://www.rsf.uni-greifswald.de/bwl/pdf/2000/01_2000.pdf Abrufdatum: 01.11.2002

Buhr P (2006): Diagnosis Related Groups (DRG) und Lebenslage. ZeS Report 1: 6-10

Bundesministerium für Gesundheit: Begründung zum Fallpauschalengesetz. BMG, Berlin 2001

DesHarnais S, Chesney J, Fleming S (1988): Trends and Regional Variations in Hospital Utilization and Quality During the First Two Years of the Prospective Payment System. Inquiry 25: 374-382

Deutsche Bundesregierung: Finanzierung, Versorgungsstrukturen und Versorgungsqualität im Krankenhausbereich nach Einführung der diagnose-bezogenen Fallpauschalen (DRG). Drucksache 16/3991. Deutscher Bundestag, Berlin 2007

Deutsche Krankenhausgesellschaft (DKG), Spitzenverbände der Krankenkassen (GKV), Verband der privaten Krankenversicherung: Allgemeine und spezielle Kodierrichtlinien für die Verschlüsselung von Krankheiten und Prozeduren. DKG, Düsseldorf 2001 


\section{LITERATURVERZEICHNIS}

Deutscher Bundesrat: Gesetzesbeschluss: Gesetz zur Einführung des diagnose-orientierten Fallpauschalensystems für Krankenhäuser (Fallpauschalengesetz - FPG). Bundesanzeiger Verlagsgesellschaft mbH, Bonn 2002

Deutsche Vereinigung für Rehabilitation Behinderter (DVfR) (2001): DRG und Rehabilitation. http://www.dvfr.de/pages/dynamic/contentShow.aspx?contentID=606 Abrufdatum: 30.10.2002

Fachgruppe Klinische PsychologInnen im Allgemeinkrankenhaus der Sektion Klinische Psychologie im Berufsverband deutscher Psychologinnen und Psychologen e.V. (BDP) (2001) : Tätigkeitsbericht 2001. http://www.sektion-klinische.de/content/downloads/FG/bericht01.DOC Abrufdatum 01.11.2002

Fitzgerald JF, Fagan LF, Terney WM (1987): Changing Patterns of Hip Fracture Care Before and after Implementation of the Prospective Payment System. JAMA 258: 218-221

Fleck LM (1987): DRGs: Justice and the Invisible Rationing of Health Care Resources. $J$ Med Philos 12: 165-191

Flintrop J (2006): Die ökomische Logik wird zum Maß der Dinge. Dtsch Arztebl 46: 2574-2577

Franz D, Thalheimer M, Krych M, Ostermann H, Krause S, Haag C, Roeder N (2008): Das DRG-System 2008 aus der Perspektive der Onkologie. Onkologe 14: 291-297

Fritze J (2002): Einführung der DRGs aus Sicht der privaten Krankenversicherung. Z aerztl Fortbildung Qual 8: 505-513

Gerety MB, Soderholm-Difatte V, Winograd C H (1989): Impact of Prospective Payment and Discharge Location at the Outcome of Hip Fracture. J Gen Intern Med 4 : 388-391

Gröticke JW, Rasche H (2001): DRGs in der Hämatologie und der Onkologie. Krankenhaus 12 : 1099-1108

Guterman S, Eggers PW, Riley G, et al. (1988): The First Three Years of Medicare Prospective Payment: An Overview. Health Care Financing Rev 9: 67-77

Hehner S, Jaeger H, Krishnan H S, Messemer J (2002): Neubeginn mit DRG: Fitness-Kur für Deutschlands Krankenhäuser McKinsey Health 1, 6-13

Jonitz G (2002): Quicker and Sicker. Kommentar zu DRGs von Dr. Günther Jonitz, Präsident der Ärztekammer Berlin. http://www.aerztekammer-berlin.de/cgi-bin/printVersion.cgi Abrufdatum 16.05 .2002 


\section{LITERATURVERZEICHNIS}

$($ Kahn et al. 1990a $)=$ Kahn KL, Rogers WH, Rubenstein LV, Sherwood MJ, Reinisch EJ, Keeler EB, Draper D, Kosecoff J, Brook RH (1990): Measuring Quality of Care with Explicit Process Criteria Before and After Implementation of the DRG-Based Prospective Payment System. JAMA 264: 1969-1973

$($ Kahn et al. 1990b) $=$ Kahn KL, Keeler EB, Sherwood MJ, Rogers WH, Draper D, Bentow SS, Reinisch EJ, Rubenstein LV, Kosecoff J, Brook RH (1990): Comparing Outcomes of Care Before and After Implementation of the DRG-Based Prospective Payment System. JAMA 264: 1984-1988

Keil L B (2001): Abenteuer DRG - Auswirkungen des neuen Vergütungssystems auf die ärztliche Arbeit. http://www.aerztekammer-berlin.de/cgi-bin/printVersion.cgi Abrufdatum: 16.05.2002

Kölking H (2001): Die Ethik der Ökonomie. DRG-Einführung. KMA Gesundheitswirtschaftsmagazin 9: 26-28

Kosecoff J, Kahn KL, Rogers W H, Reinisch EJ, Sherwood MJ, Rubenstein LV, Draper D, Roth CP, Chew C, Brook RH (1990): Prospective Payment System and Impairment at Discharge. The 'Quicker-and-Sicker' Story Revisited. JAMA 264: 1980-1983

Krause SW, Krych M, Glocker S, Franz D, Ostermann H, Ganser A, Roeder N (2005): DRG in der Hämatologie und Internistischen Onkologie: Ergebnisse aus zwei deutschlandweiten Evaluationsprojekten. Onkologie 28: 292-296

Krüger A (2002): Defizite in der Palliativmedizin durch Fallpauschalen? Hospizstiftung sieht in Tagesbudgets eine Alternative. http://www.aerztezeitung.de/docs/2002/03/04/041a0203.asp Abrufdatum: 08.08.2007

Krüger C, Rapp B (2006): Ethik im Gesundheitswesen: Behandlungsqualität - oberste Priorität. Dtsch Arztebl 103: A 320-322

Laschet H (2001): Kardiologen warnen vor Auswahl-Risiken. Ärztezeitung Online, http://www.aerztezeitung.de/docs/2001/09/27/173a0402.asp. Abrufdatum: 15.04.02

Leititis JU (2000): Rationalisierungsinstrument mit Nebenwirkungen. Ökonomische und medizinethische Spannungsfelder beim Einsatz vollpauschalierter Vergütungen. Forum Gesellschaftspolitik 2000 Juli/August: 194-197

Lenk C, Biller-Andorno N, Alt-Epping B, Anders M, Wiesemann C (2005): Ethik und Fallpauschalen. Welche Veränderungen in der Patientenversorgung sind zu erwarten? Dtsch Med Wochenschr 130:1653-1655

Macha HN (2003): Bronchialkarzinom- Epidemiologie, Diagnostik, Therapie. Internist 44: 28-34

Marckmann G: Mittelverteilung im Gesundheitswesen; in: Ethik in der Medizin. Ein Studienbuch. Wiesing U (Hrsg.) Philipp Reclam jun. Stuttgart 2004, S. 261-273 


\section{LITERATURVERZEICHNIS}

Mayer-Oakes SA, Oye RK, Leake B, Brook RH (1988): The Early Effect of Medicare's Prospective Payment System on the Use of Medical Intensive Care Services in Three Community Hospitals. JAMA 260: 3146-3149

Mayring P: Einführung in die qualitative Sozialforschung. Beltz Verlag, Weinheim 2002

Neubauer G, Nowy R (2001): DRGs in Australien - Fallkosten, Kalkulation Vergütungsfindung und $\mathrm{Zu}$ - und Abschläge. Krankenhaus 2: 123-129

Neubauer G, Nowy R, Lindl C (2001): Reduktion der Verweildauer durch DRGs. Was geschieht mit Kurzzeitfällen? Krankenhaus 12: 1093-1098

Neumann HA: Homo oeconomicus im Krankenhaus; in: Normkultur versus Nutzenkultur. Über kulturelle Kontexte von Bioethik und Biorecht, Schweidler und Hoffmann (Hrsg.). deGruyter, Berlin 2006, S.339-366

Rabbata S, Meißner M (2009): Hoppe verlangt Mut zur Wahrheit. Dtsch Arztebl 21: B 865-67

Roeder N: Anpassungsbedarf der Vergütung von Krankenhausleistungen für 2007; in: Gutachten im Auftrag der Deutschen Krankenhausgesellschaft. Deutsche Krankenhausgesellschaft, Berlin 2006

Roeder N, Fiori W, Bunzemeier H, Fürstenberg T, Hensen P, Loskamp N, Franz D, Glocker S, Wenke A, Reinecke H, Irps S, Rochell B, Borchelt M (2004): G-DRG-System 2005: Was hat sich geändert? (II). Spezifische Änderungen in medizinischen Leistungsbereichen. Krankenhaus 12:1022-1039

Rogers WH, Draper D, Kahn KL, Keeler EB, Rubenstein LV, Kosecoff J, Brook RH (1990): Quality of Care Before and After Implementation of the DRG-Based Prospective Payment System. A Summary of Effects. JAMA 264: 1989-1993

Rubenstein LV, Kahn KL, Reinisch EJ, Sherwood MJ, Rogers WH, Kamberg C, Draper D, Brook RH (1990): Changes in Quality of Care for Five Diseases Measured by Implicit Review, 1981 to 1986. JAMA 264: 1974-1979

Sager MA, Leventhal EA, Easterling DV (1987): The Impact of Medicare's Prospective Payment System on Wisconsin Nursing Homes. JAMA 257: 1762-1766

Sager MA, Easterling DV, Kindig DA, Anderson OW (1989): Changes in the Location of Death after Passage of Medicare's Prospective Payment System. New engl j med 320: 433-439

Sangha O: Adaption von DRGs in Deutschland. Institutionelle Rahmenbedingungen der Einführung eines DRG-Systems; zitiert nach Skript des Symposiums des AOK-Bundesverbandes 1999 (gehalten am 18. Mai 1999), AOK-Bundesverband, München 1999 


\section{LITERATURVERZEICHNIS}

Schnell R, Hill P, Esser E: Methoden empirischer Sozialforschung. 6. Auflage, Oldenbourg Verlag, München 1999

Schroeders v. N, Köbberling J (2002): Einfluss von Vergütungssystemen auf die medizinische Qualität. Med Klin 97: 429-433

Sitzler G (2001): DRG-Serie I: Risiko für Maximalversorger? Intensivmedizinische DRGs. KMA Gesundheitswirtschaftsmagazin 10: 50-53

Statistisches Bundesamt Deutschland (2006): Krankenhäuser: Einrichtungen, Betten und Patientenbewegung 1991-2005

http://www.destatis.de/jetspeed/portal/cms/Sites/destatis/Internet/DE/Navigation/Statistiken/Gesu ndheit/Krankenhaeuser/Krankenhaeuser.psml Abrufdatum: 07.06.2007

Swedish Health Care and Priorities Commission: No easy choice: The Difficult Priorities of Health Care. Ministry of Health and social affairs, Stockholm 1993

Tumorzentrum Ludwig Heilmeyer - Comprehensive Cancer Center Freiburg CCCF (Hrsg.): Empfehlungen zum Lungenkarzinom. 3. Auflage. Tumorzentrum Freiburg, Freiburg 2006

Veatch R (1986): DRGs and the ethical reallocation of resources. Hastings Cent Rep 16/3: 32-40

Wehkamp KH (2002): DRGs als medizinethische Herausforderung. Dtsch Med Wochenschr 127: 395-398

Wilmsen-Neumann J (2005): Stirbt die ganzheitliche Sicht des Menschen? Das deutsche DRGSystem und die Palliativmedizin. Klinikarzt 34:24-28

Zaddach M (2001): Ethik pur ist nicht zu haben. Gespräch mit Margot von Renesse. KMA Gesundheitswirtschaftsmagazin 9: 18-20

Zentrale Ethikkommission (ZEK-BÄK) (2000): Prioritäten in der medizinischen Versorgung im System der gesetzlichen Krankenversicherungen (GKV): Müssen und können wir uns entscheiden? Dtsch Arztebl 97: 1017-1023 


\section{DANKSAGUNG}

\section{DANKSAGUNG}

Ich möchte mich bei allen bedanken, die mir bei der Fertigstellung der Dissertation geholfen haben.

Mein besonderer Dank gilt Herrn PD Dr. phil. Christian Lenk, der mich während der gesamten Projektdauer betreut hat und mir jederzeit geduldig mit Rat und Tat zur Seite gestanden hat. Seine Ratschläge und Ermutigungen haben entscheidend dazu beigetragen, die Dissertation fertigzustellen.

Des Weiteren möchte ich Frau Prof. Dr. med. Claudia Wiesemann danken, die immer für mich ansprechbar war und mir mit konstruktiver Kritik geholfen hat die Dissertation in die nun vorliegende Form zu bringen.

Auch möchte ich mich bei Frau Dr. med. Katrin Radenbach, Frau Anna-Karina Jakovljevic und Herrn Dr. med. Philipp Koch bedanken, die mir bei der Durchführung der Patientenbefragung geholfen haben. Herrn Dr. med. Bernd Alt-Epping danke ich für Informationen zum stationären onkologischen Alltag und die gute Zusammenarbeit mit der Abteilung Hämatologie und Onkologie des Universitätsklinikums Göttingen.

Vielen Dank an Herrn Prof. Dr. Reinhard Hilgers für die Hilfe in Bezug auf statistische Fragen. Ebenfalls bedanke ich mich bei Herrn Dr. Bodo Sliwa, für die Bereitstellung und Aufarbeitung der Daten der Stabsstelle Controlling des Universitätsklinikums Göttingen.

Mein Dank gilt auch meinen Eltern, die mir auf dem langen Weg der Dissertation zur Seite gestanden haben und mir durch Anregungen und Korrekturen geholfen haben. Außerdem bedanke ich mich bei Frau Annette Bartlau für die letzten Korrekturen. 


\section{LEBENSLAUF}

Am 13. Januar 1981 wurde ich als erstes Kind von Karin und Hans-Peter Anders in Gronau/ Leine geboren. Mein jüngerer Bruder Stefan studiert Physik in Göttingen.

\section{Schulbildung:}

Von 1993 bis 2000 besuchte ich das Max-Planck-Gymnasium in Göttingen, an dem ich 2000 meine Hochschulreife erlangte.

\section{Studium der Humanmedizin:}

Im Wintersemester 2000 / 2001 begann ich das Studium der Humanmedizin an der GeorgAugust-Universität Göttingen. Nach Bestehen der Ärztlichen Vorprüfung im Jahr 2002 folgte der klinische Studienabschnitt. Im Jahr 2003 bestand ich den ersten Abschnitt der Ärztlichen Prüfung. Im Jahr 2006 folgte das Bestehen des zweiten Abschnitts der Ärztlichen Prüfung. Danach begann das Praktische Jahr, dessen erstes Tertial ich in der Chirurgie am Allgemeinen Krankenhaus in Wien absolvierte. Es folgten jeweils ein internistisches und ein pädiatrisches Tertial im Klinikum Oldenburg. Im April 2007 schloss ich das Studium der Humanmedizin erfolgreich mit dem Bestehen des dritten Abschnitts der Ärztlichen Prüfung ab und erhielt die ärztliche Approbation.

\section{Beruflicher Werdegang}

Von Juni bis August 2007 arbeitete ich im Institut für Ethik und Geschichte der Medizin als Wissenschaftliche Hilfskraft. Darauf folgte von September bis November 2007 eine Anstellung als Assistenzärztin in der internistischen Abteilung des Klinikums Links der Weser in Bremen.

Seit Dezember 2007 arbeite ich als Weiterbildungsassistentin der Pädiatrie im Klinikum Salzgitter/ Lebenstedt. 